

\author{
Universidad Politécnica de Madrid \\ Escuela Técnica Superior \\ de Ingenieros Informáticos
}

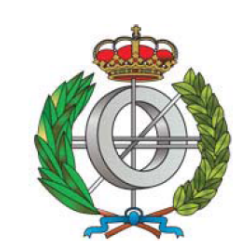

\title{
Understanding Clinical Narratives in Oncological Domain
}

\author{
Author: Marjan Najafabadipour \\ MS Computer Science \\ Supervisor: Dr. Ernestina Menasalvas Ruiz \\ PhD Computer Science
}

A thesis submitted for the degree of
Doctor of Philosophy

Madrid, September 2020 



\title{
Understanding Clinical Narratives in Oncological Domain
}

\author{
Author: Marjan Najafabadipour \\ MS Computer Science \\ Supervisor: Dr. Ernestina Menasalvas Ruiz \\ PhD Computer Science
}

\begin{abstract}
A thesis submitted for the degree of
Doctor of Philosophy
\end{abstract}

Madrid, September 2020 

The best way to predict the future is to create it. Abraham Lincoln 

I have endured hardship and never gave up. Here I stand today, thinking of the lessons I have learned because they are making me, who I am today, A Data Scientist! 

Standing at the highest stage of academic life, I can only think of my parents, who strive to give me the best opportunities in life. They have been the impetus I have ever needed to succeed.

Tamim, my soulmate and my better half. You have always cherished my dreams and supported me through ups and downs to reach this stage. 



\section{Acknowledgement}

It is a great pleasure, now that this voyage is reaching a conclusion to look back and remember all the people who helped me through the research work that led to this PhD Thesis. I would like to take this opportunity to thank every one who have contributed to the the development of this Thesis, both professionally and personally.

Going back in time, firstly, I would like to thank My Supervisor - Dr. Ernestina Menasalvas Ruiz, who has guided me to successfully complete this PhD Thesis. Your advice and wisdom has positively marked my career. You have encouraged me to participate in many different projects and to continuously study new things in the field of Data Science to become a more literate person. You have been tireless and kind in helping me to tackle difficult challenges over the course of four years.

Also, I would like to thank Dr. Ryan Brinkman and Dr. Kristofer Bouchard for supervising my research works during my $\mathrm{PhD}$ mobility programmes at The University of British Columbia in Vancouver, Canada and the University of California Berkeley in Berkeley, USA, respectively. I learnt so many valuable research methods in your teams and expanded my vision with new ideas.

Furthermore, I would like to express my gratitude to Dr. Alejandro Rodríguez-González, Dr. Massimiliano Zanin and Juan Manuel Tuñas for their contribution and help through development of this research work. You have been that lighthouse for me at Technical University of Madrid (UPM).

Finally, I would like to thank my family for their unending support throughout my research both spiritually and in general:

My Father, you are a man like no other. I appreciate all you did for me. Without the inspiration, drive, and support that you have given me, I might not be the person I am today.

My Mother, you have been my main motivation to achieve my $\mathrm{PhD}$ and I am forever grateful for all that you are and who you have taught me to be. There are not enough words to even describe how important you are in my life, and you continue to influence how I live mine. 
My Brothers - Ali and Reza, thank you for always standing by my side and valuing my goals. The best part of having brothers like you is that I have never feared losing your support even when I am living far from you.

My Cousin - Amir Reza, we came into the world like sister and brother. I appreciate your continuous support throughout my $\mathrm{PhD}$ studies and every day of my life.

My Father and Mother In-law, I am extremely grateful for your love, prayers and belief that I can successfully complete my PhD studies. Calling me your hero and pride was the main source of energy, which helped me in achieving this goal.

My Brother In-law - Halim, I must express my profound gratitude for your unfailing support during my years of research. Thank you for being someone to lean on, someone to count on and someone to tell on.

My Husband - Tamim, my acknowledgement would be incomplete without thanking you as the biggest source of my strength, who inspires me every single day of life. Thank you for being the person who always said "lets see how we can fix this" instead of "I told you so" during my PhD studies. I owe you a great deal for this. You have always cherished my dreams, goals and achievements and I am extremely honored and proud to call you my husband. 



\section{Abstract}

Digital data generation has increased rapidly in $21^{\text {st }}$ century with the explosion of data generated by the healthcare industry. The rate of clinical data generation is growing dramatically as the result of adaption of Electronic Health Records (EHRs) worldwide. EHRs are creating rich databases, containing a large amount of clinical data about the patient's medical care. Transforming clinical data into knowledge for improving patient's care has been the goal of biomedical informatics professionals for decades. However, this is still a challenging task due to the imitations of processing ability; accuracy and completeness of records; interoperability and lack of standardization; cost; security and privacy concerns; and inability to extract unstructured information in particularly, texts of EHRs.

Retrieval of textual information from clinical narratives is challenging because of: (1) limitations of ontologies to cover all the medical concepts mentioned in the texts; (2) difficulty in extraction of time expressions due to the presence of various categories, formats, and styles a time expression can be written in; existence of abbreviations; mentions of ambiguous time expression; and uncertainty for interpretation of relative time expressions; and (3) complexity in identification of temporal relations because of ungrammatical nature of clinical texts, which has a distinct sub-language, ignoring several restrictions needed in grammar for mentioning temporal relations in texts.

In oncological domain, especially for lung cancer disease, which has higher prevalence and mortality rate worldwide, the exploitation of data stored in EHRs can lead to better understanding of the treatment responses and outcomes. However, the treatment of lung cancer does not only depend on the current condition of a patient, but also on his/her past medical history. Therefore, it is very helpful for clinicians to have a complete knowledge of the patient's natural history, which includes the disease and its progression over time. The process of reconstructing a patient's natural history from EHRs requires the identification of several key elements, like medical concepts, time expressions, and temporal relations. 
Motivated by all these challenges, this Thesis focuses on the analysis of textual information written in Spanish EHRs with the ultimate goal of reconstructing the patient's natural history. Therefore, the main contributions of the Thesis are: (1) development of specific rule-based Natural Language Processing (NLP) annotators for extracting lung cancer concepts. These annotators identify tumor mutation status related to the patient's disease diagnosis, the various tumor stage codes written in laboratory test results and performance status concepts, which are used for determining the severity of patient's disease; (2) creation of a rule-based Temporal Tagger for recognition and normalization of time expressions from free texts. The performance of the temporal tagger is compared against the Spanish versions of SUTime and HeidelTime; and (3) building a Temporal Reasoning System for finding temporal relations between medical concepts and date expressions mentioned at sentence, section and document level. The Temporal Reasoning system implements a set of heuristic rules and dependency parsing technique and its performance is compared with the Spanish version of TIPSem.

Finally, this Thesis presents the validation of the proposed NLP modules in a real case study in which more than 200,000 clinical notes of patients, who were diagnosed with lung cancer are analyzed. 



\section{Resumen}

La generación de datos digitales ha aumentado rápidamente en el siglo XXI con la explosión de datos generados por la industria de la salud. La generación de datos está creciendo dramáticamente como resultado de la inserción de la historia clínica digital (HCE). La HCE está generando bases de datos enriquecidas, que contienen una gran cantidad de datos clínicos sobre la atención médica del paciente. Transformar los datos clínicos de la HCE en conocimiento para mejorar la atención del paciente ha sido el objetivo de los profesionales de la informática biomédica durante décadas. Sin embargo, esto sigue siendo una tarea desafiante debido entre otros a: i) la imitación de la capacidad de procesamiento; ii) exactitud e integridad de los registros; iii) interoperabilidad y falta de estandarización; iii) coste; iv) temas relacionados con la seguridad y privacidad; y v) por la incapacidad para extraer información de los datos no estructurados en particular los datos en lenguaje natural que contiene la HCE.

La recuperación de información textual de narrativas clínicas es una tarea difícil debido en primer lugar a la limitación de las ontologías y diccionarios para cubrir todos los conceptos médicos que se pueden mencionar en las notas clínicas. En segundo lugar, la extracción de expresiones de tiempo almacenadas en la HCE también es un reto debido a la presencia de varias categorías, formatos y estilos cuando expresamos expresiones temporales en lenguaje natural. Por otra parte, la existencia de abreviaturas; menciones de expresión temporal ambiguas generan incertidumbre para la interpretación de expresiones de tiempo en lenguaje natural. Por último, la manera en la que están escritas las notas clínicas con estructuras gramaticales que a menudo no son las tradicionales complica la interpretación de las expresiones de tiempo en textos médicos.

En el dominio oncológico, y en particular si nos referimos al cáncer de pulmón, que es el que tiene mayor tasa de prevalencia y mortalidad en todo el mundo, la explotación de los datos almacenados en los HCE podría conducir a una mejor comprensión de la enfermedad y a proporcionar respuestas sobre el tratamiento y sus resultados. Sin embargo, el tratamiento del cáncer de pulmón no solo depende de la condición actual de un paciente, sino de todo su historial médico anterior. Por lo tanto, es muy útil para los médicos tener un 
conocimiento completo de la historia natural del paciente, que incluye desde antes del diagnóstico de la enfermedad así como, su progresión. El proceso de reconstruir la historia natural de un paciente a partir de la HCE requiere de la identificación de varios elementos clave, como conceptos médicos, expresiones de tiempo y relaciones temporales entre los conceptos encontrados.

Motivados por todos estos desafíos, esta tesis se enfoca en el análisis de información textual en español de la HCE con el objetivo final de reconstruir la historia natural del paciente oncológico. Por tanto, en esta Tesis proponemos un conjunto de anotadores de procesamiento de lenguaje natural (NLP) basados en reglas para extraer conceptos de cáncer de pulmón. En particular: estadio, mutaciones, Performance Status. Por otra parte, se desarrolla un anotador temporal basado en reglas para el reconocimiento y normalización de expresiones temporales. Para terminar se presenta un sistema de razonamiento temporal para encontrar relaciones temporales entre conceptos médicos y las expresiones temporales mediante la implementación de reglas heurísticas y técnicas de análisis de dependencias.

La tesis presenta la validación de los módulos presentados en un caso de estudio real en el que se analizan mas de 200,000 notas de pacientes que han sido diagnosticados con cáncer de pulmón. 



\section{Contents}

1 Introduction 1

1.1 Introduction and Motivation . . . . . . . . . . . . . . . . . 1

1.2 Objectives and Hypothesis $\ldots \ldots \ldots \ldots$

1.3 Contributions . . . . . . . . . . . . . . . . . . 8

1.4 Publications, International Research Mobility Programmes,

and Awards . . . . . . . . . . . . . . . . . . . . 9

1.4.1 Publications . . . . . . . . . . . . . . . . . . . 9 9

1.4 .2 International Research Mobility Programmes . . . . . . 10

1.4 .3 Awards . . . . . . . . . . . . . . . . 10

1.5 Structure of the Thesis $\ldots \ldots \ldots$. . . . . . . . . . . . . 11

2 Lung Cancer Concept Annotation from Spanish Clinical Narratives 13

2.1 Introduction . . . . . . . . . . . . . . . . . . . 13

2.2 Problem Statement . . . . . . . . . . . . . . . . . . . . 16

2.3 State of the Art . . . . . . . . . . . . . . . 16

$2.3 .1 \quad$ NLP Preliminaries . . . . . . . . . . . . . . . . . . 17

$2.3 .2 \quad$ Frameworks for Building Annotators . . . . . . . . . 17

2.3.3 Extraction of Information from Free Texts of EHRs . . 20

2.4 Solution . . . . . . . . . . . . . . . . . . . . . . 30

2.4 .1 Tumor Mutation Status Recognition . . . . . . . . . 32

2.4 .2 Tumor Stage Recognition . . . . . . . . . . . . . . . 34

2.4 .3 Performance Status Recognition . . . . . . . . . . . . . 35

2.4.4 The Process of Annotations Output Generation . . . . 36

2.5 Validation $\ldots \ldots \ldots \ldots \ldots$

2.5 .1 Dataset . . . . . . . . . . . . . . . . . . 39

2.5 .2 Experiments . . . . . . . . . . . . . . . . . 44

2.5 .3 Results . . . . . . . . . . . . . . . . . . . . . . . 45 
2.6 Discussion $\ldots \ldots \ldots \ldots$. . . . . . . . . . . . . . . . . . . . . 47

\section{Recognition of Time Expressions in Spanish Electronic Health} $\begin{array}{ll}\text { Records } & 49\end{array}$

3.1 Introduction . . . . . . . . . . . . . . . . . 50

3.2 Problem Statement . . . . . . . . . . . . . . . . . . . . 51

3.3 State of the Art . . . . . . . . . . . . . . . . . 52

3.3.1 NLP Systems for Annotation of Time Expression from Free Texts . . . . . . . . . . . . . . . 52

3.3.2 NLP Systems for Annotation of Time Expression from Clinical Texts . . . . . . . . . . . . . . 55

3.3 .3 Preliminaries . . . . . . . . . . . . . . . 57

3.4 Solution . . . . . . . . . . . . . . . . . . . . 58

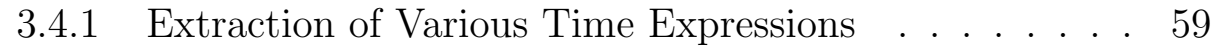

3.4.2 Filtration of Time Expressions . . . . . . . . . . . . . . 60

3.4.3 Resolution of Time Expressions with Respect to a Reference Date . . . . . . . . . . . . . . . 60

3.4.4 Normalization of Time Expressions to a Standard Date Format................. . 60

3.5 Validation . . . . . . . . . . . . . . . . . 61

3.5.1 Dataset . . . . . . . . . . . . . . . 62

3.5.2 Experiments . . . . . . . . . . . . . 63

3.5.3 Results . . . . . . . . . . . . . . . . . . . . . 64

3.6 Discussion . . . . . . . . . . . . . . . . . . . . 66

4 Reconstructing the Patient's Natural History from Electronic

Health Records $\quad 68$

4.1 Introduction . . . . . . . . . . . . . . . . . . . . . . . . . . . . 69

4.2 Problem Statement . . . . . . . . . . . . . . . . . . . . . 73

4.2.1 Challenge 1 - Linkage of Medical Events to Time Expressions . . . . . . . . . . . . . . . . . . . 73

4.2 .2 Challenge 2 - Derivation of the Order of Medical Events from Patient's EHRs . . . . . . . . . . . . 74

4.3 State of the Art . . . . . . . . . . . . . . . . . . . . 75

4.3.1 Definition of Event in General Domain VS Medical Domain . . . . . . . . . . . . . 75

4.3 .2 Temporal Reasoning . . . . . . . . . . . . . 76 
4.3.3 Temporal Ordering of Medical Events Across English EHRS ................... 85

4.4 Solution . . . . . . . . . . . . . . . . . . . . . 87

4.4 .1 Temporal Reasoning System . . . . . . . . . . . . . . . 89

4.4 .2 Timeline Constructor . . . . . . . . . . . . . . . . . . . 95

4.5 Validation . . . . . . . . . . . . . . . . . . 97

4.5.1 Sample Dataset Selection for First Use Case . . . . . . 98

4.5 .2 Experiments . . . . . . . . . . . . . . . . . 99

4.5 .3 Results . . . . . . . . . . . . . . . . 100

4.6 Discussion $\ldots \ldots \ldots$. . . . . . . . . . . . . . . . . . . . . 103

\begin{tabular}{|lll}
5 & Implementation and Deployment & 105
\end{tabular}

5.1 Introduction ..................... 105

5.2 Identifying the Temporal Order of Medical Events Across EHRs 106

$5.2 .1 \quad$ Problem Analysis . . . . . . . . . . . . . . . 106

5.2 .2 Design of the Solution . . . . . . . . . . . . . . . 108

5.3 Deployment ....................... . . 111

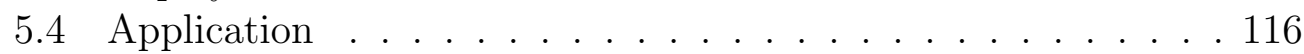

5.5 Discussion . . . . . . . . . . . . . . . . . . . . . . 118

6 Conclusions and Future Work 123

6.1 Conclusions . . . . . . . . . . . . . . . . . 123

6.2 Future Lines . . . . . . . . . . . . . . . . . . . . . . 125

\begin{tabular}{lr}
\hline Bibliography & 127
\end{tabular} 



\section{List of Figures}

1.1 Annual size of Global Datasphere predicted by IDC 2018 [1] . 2

$2.1 \quad$ A typical NLP pipeline $[2]$. . . . . . . . . . . . . . . . . . . 17

2.2 UIMA frameworks, infrastructure and components [3] . . . . . 19

$2.3 \quad$ UIMA architecture $[4 \| \ldots \ldots \ldots . \ldots . \ldots . \ldots 20$

2.4 The distribution of NLP publications in medical domain from 1994 through 2020 . . . . . . . . . . . . . . 22

2.5 Comparison of the number of NLP publications in medical domain for English versus Spanish from 1994 through 2020 . . 23

2.6 The annotation results of EGFR, ALK and ROS1 annotators

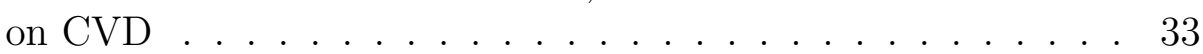

2.7 AJCC 8th edition - lung cancer stage grouping and TNM

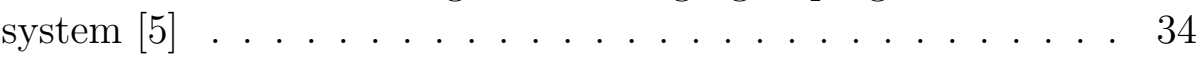

2.8 The annotation results of Stage and TNM annotators on CVD 35

2.9 The annotation results of ECOG and Karnofsky annotators on

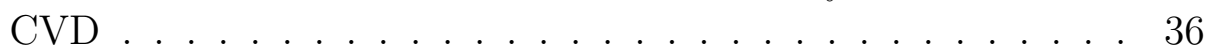

2.10 Processing engine architecture . . . . . . . . . . . . . . 37

2.11 XMI annotation results on UIMA annotation viewer. . . . . . . 38

2.12 Process of clinical notes and reports generation for a patient in HUPHM . . . . . . . . . . . . . . . . . . . . . . . . . . . . 40

2.13 Example of a clinical note structure . . . . . . . . . . . . . . . 41

2.14 Example of a clinical report structure . . . . . . . . . . . . . . 42

3.1 Comparison of the results of our Temporal Tagger with SUTime and HeidelTime . . . . . . . . . . . . . . . . . . . . 65

4.1 An example of a natural history of a patient suffering from lung cancer (top part), and the corresponding medical timeline for disease diagnosis and progression (bottom part) $\ldots . . .69$ 
$4.2 \quad$ Architecture of the proposed framework . . . . . . . . . . . . . 88

$4.3 \quad$ Example of dependency parsing by UDpipe . . . . . . . . . . . . 90

4.4 Dependency parse tree analysis for an example that satisfies Rule $-1[6] \mid \ldots \ldots \ldots \ldots . \ldots \ldots 3$

4.5 Dependency parse tree analysis for an example that does not

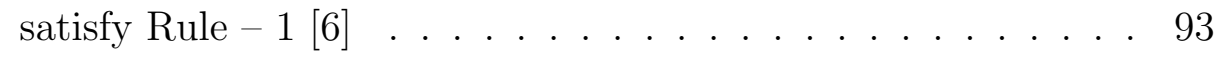

$4.6 \quad$ Dependency parse tree analysis for an example that satisfies

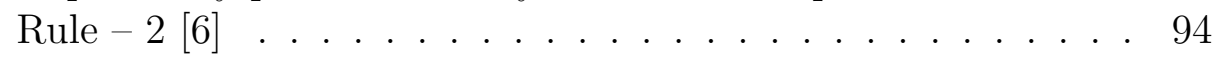

4.7 Dependency parse tree analysis for an example that does not

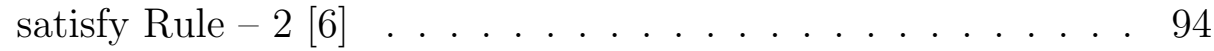

4.8 Comparison of the results of our Temporal Reasoning System with TIPSem . . . . . . . . . . . . . . . . 102

5.1 Snapshot of the structured diagnosis events for a patient in the Document database . . . . . . . . . . . . . . . . . . . 107

5.2 Snapshot of the structured stage grouping events for a patient in the Document database . . . . . . . . . . . . . . . . 107

5.3 Snapshot of the structured TNM events for a patient in the Document database . . . . . . . . . . . . . . . 107

5.4 Snapshot of the structured information about the patients and their EHRs in the Document database . . . . . . . . . . . . . 108

5.5 Snapshot of Document database design containing annotations 109

$5.6 \quad$ Flow chart for identifying the patient's diagnosis date . . . . . 110

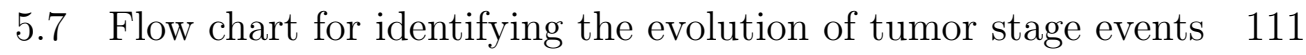

5.8 Technologies used to deploy our Framework . . . . . . . . . . 112

5.9 Overview of the production framework . . . . . . . . . . . . 117

5.10 Login dashboard of iASiS platform . . . . . . . . . . . . . . . 118

5.11 Analytics dashboard of iASiS platform . . . . . . . . . . . . . . 119

5.12 An Example of population selection and results generation in analytics dashboard . . . . . . . . . . . . . . . . . 120

5.13 Selection of parameters to access bibliographical links and the survival probability image . . . . . . . . . . . . . 121

5.14 Survival probability of lung cancer patients based on the parameter of tumor stage . . . . . . . . . . . . . . 121 



\section{List of Tables}

$2.1 \quad$ Details of the selected samples for conducting experiments . . 43

2.2 Validation results . . . . . . . . . . . . . . . . . . 46

3.1 Example of time expressions annotated by our Temporal Tagger (with 2016-12-23 as reference date) . . . . . . . . . . . . . . 61

$4.1 \quad$ Examples for rules of temporal relation identification . . . . . 92 



\section{Listings}

$2.1 \quad$ Pseudocode of algorithm for rule-based annotators . . . . . . . 31

$4.1 \quad$ Pseudocode of Time-line Constructor . . . . . . . . . . . . . . 95

$5.1 \quad$ Example of making a HTTP Post request to our NLP framework 113

5.2 Example of making a HTTP GET request to our NLP framework 113 



\section{Acronyms}

ACE Automated Content Extraction

ACL Association for Computational Lingustics

ACM Association for Computing Machinery

AEs Analysis Engines

AJCC American Joint Committee on Cancer

ALK Anaplastic Lymphoma Kinase

API Application Programming Interface

ARC Automated Retrieval Console

BNs Bayesian Networks

CaFE Case Finding Engine

CAS Common Analysis Structure

caTIES cancer Tissue Information Extraction System

CDKRM Cancer Disease Knowledge Representation Model

CLAMP Clinical Language Annotation, Modeling, and Processing

C-liKES Clinical Knowledge Extraction System

CPE Collection Processing Engine

CRF Conditional Random Field

cTAKES Clinical Text Analysis and Knowledge Extraction System

CTB Center for Biomedical Technology

CVC Central Venous Catheter

CVD CAS Visual Debugger 
DANTE Detection And Normalization of Temporal Expressions

DNA Deoxyribonucleic Acid

ECOG Eastern Cooperative Oncology Group

EGFR Epidermal Growth Factor Receptor

EHRs Electronic Health Records

FN Fasle Negative

FP False Positive

GATE General Architecture for Text Engineering

GROK Grammatical Representation of Objective Knowledge

HITEx Health Information Text Extraction

HUPHM Hospital Universitario Puerta de Hierro-Majadahonda

H2A Human Health Analytics

iASiS Integration and analysis of heterogeneous big data for precision medicine and suggested treatments for different types of patients

IASLC International Association for the Study of Lung Cancer

IBM International Business Machines

ICD-9-CM International Classification of Diseases-Ninth Revision-Clinical Modification

ICHOM International Consortium for Health Outcomes Measurement

IDC International Data Corporation

IE Information Extraction

IMIA International Medical Informatics Association

ISO International Organization for Standardization

IT Information Technology 
i2b2 Integrating Biology and the Bedside

JSON JavaScript Object Notation

KMCI KnowledgeMap Concept Indexer

LR Logistic Regression

LSP Linguistic String Project

MedEx Medication Extraction

MedLEE Medical Language Extraction and Encoding System

MEDLINE Medical Literature Analysis and Retrieval System Online

MedTAS Medical Text Analysis System

MedXN Medication Extraction and Normalization

MeSH Medical Subject Headings

MIDAS Data Mining and Simulation

ML Machine Learning

MLP Medical Language Processing

MSE Mean Squared Error

MUC Message Understanding Conference

M+ MPLUS

NER Named Entity Recognition

NEs Named Entities

NLP Natural Language Processing

NLUS Natural Language Understanding System

NSCLC Non-Small Cell Lung Cancer

POA Pairwise Ordering Accuracy 
PoS Part of Speech

PS Performance Status

QA Query and Answering

RNN Recurrent Neural Network

RxCUI RxNorm Concept Unique Identifier

SCLC Small Cell Lung Cancer

SNOMED Systematized Nomenclature of Medicine

STCSP Simple Temporal Constraint Stratification Problem

STP Simple Temporal Problem

SVM Support Vector Machine

SymText Symbolic Text Processor

SynTime Syntactic Time

TEER Temporal Expression ExtractoR

TERN Time Expression Recognition and Normalization

THYME Temporal Histories for Your Medical Events

TIDA Text and Images Data Analyzer

TIPSem Temporal Information Processing based on Semantic information

TIPSemB-F TIPSemB-Freeling

TP True Positive

UDPipe Universal Dependency Pipe

UIM Unstructured Information Management

UIMA Unstructured Information Management Architecture

UMLS Unified Medical Language System 
UPM Universidad Politécnica de Madrid

USA United States of America

WFST Weight Finite-State Transducer

WHO World Health Organization

XMI XML Metadata Interchange

ZBs Zettabytes 



\section{CHAPTER}

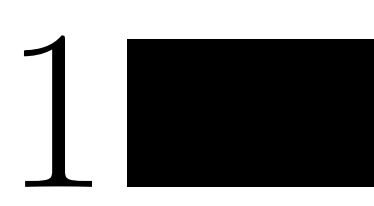

\section{Introduction}

The automatic retrieval of a patient's natural history from Electronic Health Records (EHRs) can support clinicians in their decision making. To extract the natural history, recognition of medical concepts, extraction and normalization of time expressions, and identification of temporal relations from EHRs is required. However, these tasks are hard to tackle due to the writing quality and unstructured (free texts format) nature of information stored in EHRs. Therefore, in this Thesis, we analyze textual clinical data and propose new Natural Language Processing (NLP) techniques for extraction of lung cancer medical concepts, time expressions and temporal relations written in Spanish EHRs.

\subsection{Introduction and Motivation}

Information Technology (IT) is experiencing a significant change in $21^{\text {st }}$ century due to the rise of internet as well as digital devices and systems such as computers, laptops, cellphones, robots, autonomous vehicles, etc. As existing technologies are becoming cheaper and more accessible to different kind of users and as these users are transforming from consumers to the so called prosumers, who do not only consume but also produce data, an enormous amount of digital date is being generated everyday. 
Chapter 1. Introduction

1.1. Introduction and Motivation

According to the International Business Machines (IBM) [7], people are generating 2.5 quintillion bytes of digital data every day. In addition, International Data Corporation (IDC) 2018 [1] predicted that the Global Datasphere will grow from 33 Zettabytes (ZBs) in 2018 to 175 ZBs by 2025 (Figure 1.1).

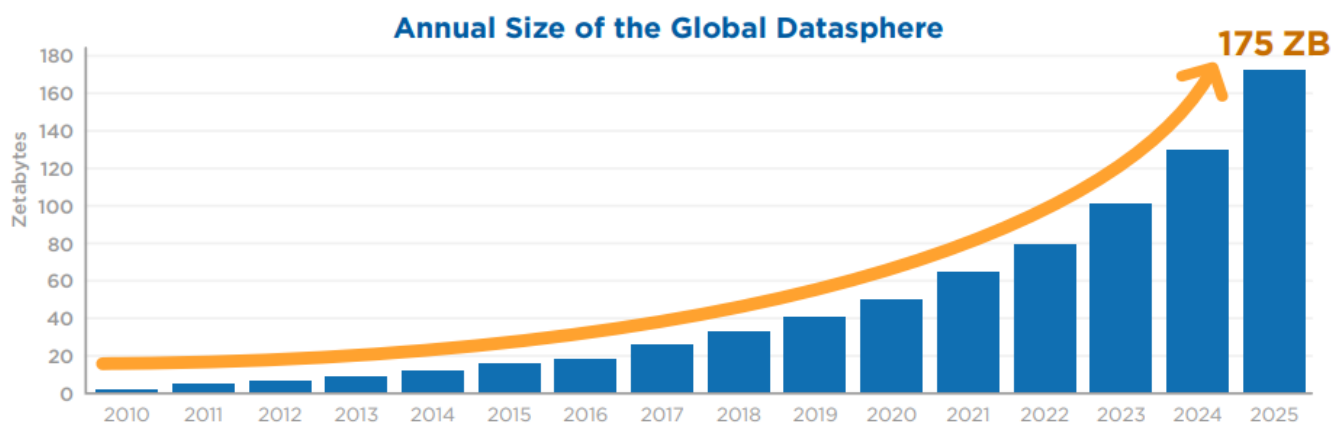

Figure 1.1: Annual size of Global Datasphere predicted by IDC 2018 |1

The explosion of data generated by the healthcare industry - laboratory tests, emergency and discharge reports, medical images gathered from scans, etc. - is also influencing the amount of digital data generated. IDC 2018 declared that among all the industries, healthcare is facing the fastest growth of data generated and it's growth is predicted to rise by $36 \%$ from 2018 to 2025. As mentioned in [8], the healthcare organizations accumulated $85 \%$ more amounts of data in 2019 than they did two years before it.

Towards the digitization of the society, hospitals, clinics and health centers are storing patient's information in unstructured (i.e. containing texts and images) clinical documents, what is called as Electronic Health Records (EHRs). The adaption of EHRs is generally expected to improve and increase the quality of healthcare, decrease medical care costs and provide patients with more involvement in their medical care [9].

As a result, the healthcare industry has expressed a growing interest and is expected to continue raising the adaption of EHRs [10]. In 2004, the office-based clinicians at hospitals of United States of America (USA) adapted EHRs precisely at the rate of $20.8 \%$. This ratio was increased dramatically to $85.9 \%$ in 2017 [11]. In addition, according to a global study performed 
Chapter 1. Introduction

1.1. Introduction and Motivation

in [12] in 2013, Spain was in the second position in the adaption of EHRs with the ratio of $72 \%$ in 2011 . This rate was increased slightly to $73 \%$ in 2012. We are aware that this information are quite old, however, to the best of our knowledge, no new information has been published about the adaption of EHRs in Spain for the recent years.

While data analytics relies on the availability, accessibility and privacy of EHRs, analysis of the Big Data stored in EHRs will directly benefit healthcare industries. These benefits are [13]:

1. Diagnostics: to identify causes of illness.

2. Preventive Medicine: to prevent diseases.

3. Precision Medicine: to drive hyper-personalized care.

4. Medical Research: to cure diseases and discover new treatments and medicine.

5. Reduction of Adverse Medical Events: to spot medication errors and flag potential adverse reactions.

6. Cost Reduction: to derive better patient outcomes for long term savings.

7. Population Health: to identify disease trends and health strategies based on demographics, socio-economics and geography.

However, these benefits cannot be achieved directly as technology is not mature enough for performing data analytics due to the lack of inter-operable data schemes, lack of standards and the necessity of textual and image processing tools.

In recent years, advancing text analysis methods and their application in medical domain have received a great attention due to the large amount of textual data now available in EHRs [14]. Therefore, in this Thesis, we focus on the analysis of textual information stored in Spanish EHRs with the ultimate goal of proposing a method to reconstruct the natural history of patients. Other aspects such as interoperability, privacy, ethics and image analysis are out of scope of this work. 
Chapter 1. Introduction

1.1. Introduction and Motivation

Retrieving the patient's natural history improves clinical document summarization [15], clinical trial recruitment [16], clinical decision making [17] and patient's survival time calculation [18]. Furthermore, it allows clinicians to evaluate the quality of the provided healthcare, and to find those clinical steps, which require a special attention. To reconstruct such natural history on a medical timeline, extraction of medical concepts, time expressions and temporal relations is required.

However, retrieval of this information from clinical texts is challenging due to first of all, the unstructured nature of information locked in EHRs and the limitations of ontologies, taxonomies and controlled vocabularies to provide all the medical concepts, in particularity for lung cancer domain.

Secondly, annotation of time expressions from EHRs is difficult because of: (1) presence of three categories of natural, conventional and professional time expressions, each representing their own idiosyncrasies; (2) existence of domain specific, non-standard and abbreviated time variables; (3) mention of ambiguous time expressions having more than one meaning; and (4) uncertainty for interpretation of relative time expressions.

Moreover, clinical texts are also ungrammatical, complex and exhibit a distinct sub-language, which ignores many restrictions needed in grammar to mention temporal relations. These make automatic temporal reasoning a very difficult task.

In order to tackle the above mentioned challenges, NLP techniques have to be developed. NLP is a subfield of Computer Science, Information Engineering and Artificial Intelligence, which makes computers able to convert text into a structured representation and thus, enables computers to understand the meaning of human natural language [19].

Although in the last few years, the research community of computer science is focusing its efforts to create common methods and structures to perform analysis of textual data independent of the language, one of the main problems that NLP still faces today is the English-centric developments in many domains, in particularly in medical field. Despite of the fact that Spanish language is ranked as the second most popular language in the world with 
Chapter 1. Introduction

1.1. Introduction and Motivation

more than 572 million native speakers [20], there are only few NLP systems to extract information from clinical texts, written in this language $2,21-23]$. In addition, to the best of our knowledge, no system has been presented for tackling the problem of reconstructing the patient's natural history from Spanish EHRs.

Therefore, in what follows, we will describe the NLP techniques developed to overcome the aforementioned challenges associated with building the patient's natural history. To addresses the problem of medical concept recognition, we have developed a set of rule-based annotators over Unstructured Information Management Architecture (UIMA) framework [3,24]. UIMA is an architecture, introduced by IBM and is used as a common platform to structure various kinds of unstructured data like, texts, images, voices and videos. In addition, to process EHRs with these annotators, we have implemented them under a single Processing Engine and we have explained in this Thesis, the details of annotation results generation and their storage in XML Metadata Interchange (XMI) files and a MySQL relational database from which the Query and Answering (QA) can be followed.

In case of time expression extraction and normalization, a rule-based Temporal Tagger has been developed over UIMA framework. In addition, a comparison of the performance of our developed Temporal Tagger with the Spanish versions of SUTime (developed by the Stanford University) 25, 26 and HeidelTime (Heidelberg University) [27,28] has also been performed.

The Thesis also addresses the problem of temporal relation identification between medical concepts and time expressions from free texts of EHRs by presenting a Temporal Reasoning System. This system has been developed using Universal Dependency Pipe (UDPipe) [29,30] to build the dependency parse trees for each sentences of clinical texts and a set of rules to identify temporal relations. Furthermore, the comparison of our Temporal Reasoning System with the Spanish version of TIPSem is also performed [31].

This research has been performed at Data Mining and Simulation (MIDAS) research group, located at Center for Biomedical Technology (CTB) of Universidad Politécnica de Madrid ( $(\mathrm{UPM})$. A tight collaboration with 
Chapter 1. Introduction

1.2. Objectives and Hypothesis

Hospital Universitario Puerta de Hierro-Majadahonda (HUPHM) was created in the frame of the "Integration and analysis of heterogeneous big data for precision medicine and suggested treatments for different types of patients (iASiS)" project [32] in the development of this research by giving knowledge and data in the domain of oncology. The involvement of Computer Science domain experts and the clinicians of HUPHM have been the key factors for the development of this Thesis.

\subsection{Objectives and Hypothesis}

The main goal of the Thesis is:

"To develop a method for reconstructing the natural histories of lung cancer patients from Spanish written EHRs."

In order to fulfill this goal the detection of medical concepts, time expressions and temporal relations from clinical texts is essential. For this purpose, the following sub-goals have to be achieved:

Objective 1: To annotate medical concepts from clinical texts. In particularly, we concentrate on recognition of tumor mutation status, tumor stage and performance status related to lung cancer domain.

Research Question 1: Can medical concepts written in natural language be automatically annotated?

In the analysis of clinical texts, it is common to use NLP techniques with ontologies, taxonomies or controlled vocabularies to recognize, encode and structure medical terminologies. Clearly, this approach has a major drawback: failure in recognition of many medical concepts. This failure is due to the reason that clinicians do not always follow the standard systems for mentioning medical concepts in clinical texts and also, the lack of vocabularies to support complex medical concepts, in particularly for lung cancer domain. In Chapter 2, we will tackle this problem by developing NLP rule-based 
Chapter 1. Introduction

1.2. Objectives and Hypothesis

techniques. These are more accurate than machine learning ones as they are designed using a set of hand-crafted rule, which are based on the clinician knowledge and experience.

Hypothesis 1: The NLP rule-based techniques can be used to detect medical concepts written in natural language.

Objective 2: To extract and normalize time expression from free texts.

Research Question 2: Can time expressions mentioned in free texts be transformed into a structured and normalized format?

Machine learning and rule-based approaches of NLP can be of utmost importance in extraction and normalization of time expressions from free texts. The former requires annotated corpora, whose construction is costly and time-consuming. Also, the small size of corpora can significantly affect the processing quality. In Chapter 3, we propose the use of rule-based techniques for building a Temporal Tagger to annotate time expressions.

Hypothesis 2: The NLP rule-based techniques can be used to recognize and normalize time expressions written in natural language.

Objective 3: To identify temporal relations between medical concepts and time expressions mentioned in clinical documents.

Research Question 3: Can temporal relations between medical concepts and time expressions be identified from EHRs?

Temporal reasoning is the task that aims to identify relations between medical concepts and time expressions from free texts 33]. Current existing methods of temporal relation extraction can be classified into three categories: (1) rulebased, which extracts relations by utilizing hand-crafted inference rules; (2) kernel-based, which treats the temporal relation extraction as a classification problem with a kernel machine (e.g., Support Vector Machine (SVM)); and (3) sequence-labeling method, which is regarding temporal relation identification 
Chapter 1. Introduction

1.3. Contributions

as the sequence labeling problem (e.g., maximum entropy and Conditional Random Field ( $(\mathrm{CRF})$ ). The main draw back of second and third categories is that they implement their annotation with the help of annotated corpora. The creation of such corpora is firstly, costly and time consuming, and secondly, their completeness directly affects the processing quality. Also, the advantage of a rule-based method over a statistical machine learning approach is that rules can contain more semantic information than features used in statistical methods. Our approach consists of a rule-based method combined with dependency parsing. In Chapter 4, we identify Temporal relations by firstly, building the dependency parse trees for each sentence in the clinical texts and then, implementing a set of rules.

Hypothesis 3: A Dependency parsing technique combined with an NLP rule-based approach can be used for linking medical concepts to time expressions in clinical documents.

\subsection{Contributions}

The contributions featured in the development of the Thesis are:

- The creation of specific annotations for lung cancer domain information written in Spanish. These annotations include the concepts for tumor mutation status mentioned in the diagnosis for a patient, the various tumor stage codes obtained from laboratory tests, and more significantly, the values of different performance status scales used for determining the severity of the patient's disease. The tumor mutation status and performance status annotators are inevitably dependent on lung cancer domain in which the application is deployed, whereas the tumor stage annotators are developed general enough so that they can be used in various oncological domains.

- The design and development of a Temporal Tagger based on the UIMA framework to extract and normalize time expressions appeared in the Spanish free texts. This module is domain independent and can be used 
Chapter 1. Introduction

1.4. Publications, International Research Mobility Programmes, and Awards

for a general purpose, ensuring that the effort made in its development will make the module usable in many different contexts.

- The creation of a Temporal Reasoning System to be applied in the healthcare domain, which allows the identification of temporal relations between medical concepts and time expressions appeared at sentence, section or document levels of EHRs, written in Spanish. This system is developed by utilizing the dependency parse trees at sentence granularity, generated from UDPipe and a set of rules for finding temporal relations. Although the main use case of the system is for the medical domain, it provides a generic environment that can be used in other domains without any necessary adaptions.

\subsection{Publications, International Research Mo- bility Programmes, and Awards}

The following subsections provide the details of published papers, conducted research mobilities and received awards.

\subsubsection{Publications}

The results produced in this Thesis are documented in the following publications:

- Marjan Najafabadipour, Juan Manuel Tuñas, Alejandro RodríguezGonzález, and Ernestina Menasalvas. Lung Cancer Concept Annotation from Spanish Clinical Narratives. In Proceedings of Data Integration in the Life Sciences (DILS 2018). Lecture Notes in Computer Science, Springer International Publishing. Volume 11371. Pages 153-163. 2019. DOI: 10.1007/978-3-030-06016-9_15.

- Marjan Najafabadipour, Massimiliano Zanin, Alejandro RodríguezGonzález, Consuelo Gonzalo-Martín, Beatriz Nuñez García, Virginia Calvo, Juan Luis Cruz Bermudez, Mariano Provencio, Ernestina Menasalvas. Recognition of Time Expressions in Spanish Electronic Health 
Chapter 1. Introduction

1.4. Publications, International Research Mobility Programmes, and Awards

Records. In Proceedings of 2019 IEEE 32nd International Symposium on Computer-Based Medical Systems (CBMS 2019). IEEE. Pages: 69-74. 2019. DOI: 10.1109/CBMS.2019.00025.

- Marjan Najafabadipour, Juan Manuel Tuñas, Alejandro RodríguezGonzález, and Ernestina Menasalvas. Analysis of Electronic Health Records to Identify the Patient's Treatment Lines: Challenges and Opportunities. In Proceedings of International Conference on Innovative Techniques and Applications of Artificial Intelligence (SGAI2019). Lecture Notes in Computer Science, Springer International Publishing. Volume: 11927. Pages. 437-442. 2019. DOI: 10.1007/978-3-030-34885$4 \_33$.

- Marjan Najafabadipour, Massimiliano Zanin, Alejandro RodríguezGonzález, Maria Torrente, Beatriz Nuñez García, Juan Luis Cruz Bermudez, Mariano Provencio, and Ernestina Menasalvas. Reconstructing the Patient's Natural History from Electronic Health Records. Artificial Intelligence in Medicine. Volume: 105. 2020. DOI: 10.1016j. artmed.2020.101860

\subsubsection{International Research Mobility Programmes}

During the development of the Thesis, the author conducted two international research mobility programmes in order to be opt for the international $\mathrm{PhD}$ mention at the end of the $\mathrm{PhD}$ studies. These programmes were completed at:

- The University of British Columbia in Vancouver, Canada during which, the author collaborated with the team in design, development and validation of a clustering algorithm, which can identify homogenous samples in the flow and mass cytometry datasets.

- University of California, Berkeley in Berkeley, USA during which, the author engaged in the development and validation of classification algorithms for analysis of human sound data.

\subsubsection{Awards}

The following awards are received during the development of the Thesis: 
Chapter 1. Introduction

1.5. Structure of the Thesis

- Mobility Grant of Research in University of North America (PDI) 2019 Award: is received from Technical University of Madrid (UPM) for conducting an international research mobility programme at University of California, Berkeley in Berkely, USA.

- Mobility Grant of I+D+i of the Technical University of Madrid (UPM) 2019 Award: is received for conducting an international research mobility programme at The University of British Columbia in Vancouver, Canada.

- Predoctoral Credential of Propio Programme of I+D+i 2017: in recognition of academic excellence, the award of Predoctoral Credential of Propio Programme of $\mathrm{I}+\mathrm{D}+\mathrm{i}$ of the Technical University of Madrid (UPM) 2017 is received.

- Predoctoral Full-funded Scholarship of Propio Programme of I+D $+\mathrm{i}$ of the Technical University of Madrid (UPM) 2017 Award: is received for developing a $\mathrm{PhD}$ Thesis with the title of "Understanding Clinical Narratives in Oncological Domain" at CTB, UPM.

\subsection{Structure of the Thesis}

The rest of the Thesis is organized as follows:

- In Chapter 2, the main specific challenges in the application of NLP to annotate tumor mutation status, tumor stage and performance status related to lung cancer domain are analyzed. In addition, the design of an NLP pipeline for processing EHRs using these annotators is discussed.

- In Chapter 3, the Temporal Tagger, a domain independent annotator for extraction and normalization of time expressions from Spanish textual documents is explained.

- In Chapter 4, the specific challenges for identification of temporal relations between medical concepts and time expressions written in clinical documents are tackled through the development of a Temporal 
Chapter 1. Introduction

1.5. Structure of the Thesis

Reasoning System. In addition, an NLP framework is presented for reconstructing the natural histories of lung cancer patients form their EHRs.

- In Chapter 5, the implementation details, deployment technologies and an example of the application of our NLP framework is explained.

- Finally, in Chapter 6, the conclusions and future possible research topics are discussed. 


\section{CHAPTER}

\section{Lung Cancer Concept Annotation from Spanish Clinical Narratives}

Recent rapid increase in the generation of clinical data and rapid development of computational science make us able to extract new insights from massive datasets in the healthcare industry. Oncological EHRs are creating rich databases for documenting patient's history and they potentially contain a lot of patterns that can help in the better management of the disease. However, these patterns are locked within the free text (unstructured) portions of EHRs and consequence in limiting health professionals to extract useful information from them and to finally perform the QA process in an accurate way. The Information Extraction (IE) process requires NLP techniques to assign semantics to these patterns. Therefore, in this Chapter, we analyze the design of annotators for specific lung cancer concepts that can be integrated over UIMA framework. In addition, we explain the details of the generation and the storage of annotation outcomes.

\subsection{Introduction}

Cancer is still one of the major public health issues, ranked with the second leading cause of death globally [34]. Across Europe, lung cancer was estimated with $20.8 \%$ (over 266,000 persons) of all cancer deaths in 2011 [35] and the highest economic cost of $15 \%$ (18.8 billion) of overall cancer cost in 2009 [36. 
Chapter 2. Lung Cancer Concept Annotation from Spanish Clinical Narratives 2.1. Introduction

Early diagnosis of cancer decreases its mortality rate [37]. Hence, a great attention on the diagnosis is a key factor for both the effective control of the disease as well as the design of treatment plans.

Classically, the treatment decisions on lung cancer patients have been based upon the histology of the tumor. According to World Health Organization (WHO), there are two broad histological subtypes of lung cancer: (1) Small Cell Lung Cancer (SCLC); and (2) Non-Small Cell Lung Cancer (NSCLC) [38]. NSCLC can be further defined at the molecular level by the recurrent driver mutations [39] where mutations refer to any changes in the Deoxyribonucleic Acid (DNA) sequence of a cell [40]. The tumor mutations can occur in multiple oncogenes, including Epidermal Growth Factor Receptor (EGFR), Anaplastic Lymphoma Kinase (ALK), and Ros1 proto-oncogene receptor tyrosine kinase [41]. These oncogenes are receptor tyrosine kinases, which can activate the pathways associated with the cell growth and the proliferation 42,44 .

One of the preliminary diagnosis factor of a cancer is its tumor stage. This factor plays a significant role on making decisions for developing treatment plans. The American Joint Committee on Cancer (AJCC) manual [5, 45 specifies two standard systems for measuring the cancer stage: (1) Stage Grouping and (2) TNM. The Stage Grouping system encodes the tumor stages using roman numerals, mixed with alphabets and numbers whereas the TNM system makes use of three parameters: (1) the size of tumor (T); (2) the number of lymph nodes $(\mathrm{N})$; and (3) the presence of metastasis (M).

According to the International Consortium for Health Outcomes Measurement (ICHOM $)$, Performance Status $(\overline{\mathrm{PS}})$ is a strong individual predictor of survival in lung cancer [46]. The ICHOM working group recommended measuring PS as part of diagnosis per the Eastern Cooperative Oncology Group (ECOG). In addition to ECOG, Karnofsky is another scale for measuring PS [47]. These scales are used by doctors and researchers to assess the progress of a patient's disease, the effects of the disease on daily and living abilities of a patient and to determine an appropriate treatment and prognosis [48. 
Chapter 2. Lung Cancer Concept Annotation from Spanish Clinical Narratives 2.1. Introduction

Towards the digitization of medical data, these data have been stored in computerized medical records, named Electronic Health Records (EHRs). EHRs are rich clinical documents containing information about diagnoses, treatments, laboratory results, discharge summaries, to name a few, which can be used to support clinical decision support systems and allow clinical and translational research.

To encode, structure and extract information from EHRs, an NLP system for which the Named Entity Recognition (NER) process is its paramount task, is required. The NER process performs two tasks: (1) identification of the concepts from free texts; and (2) classification of the identified concepts into a set of predefined categories. There are two main approaches for performing NER process [49]:

1. Rule-based: this approach detects Named Entities (NEs) from free texts by using a set of hand-crafted rules. Rule-based approaches for performing NER process through means of knowledge engineering are very accurate since they are based on the physician's knowledge and experience.

2. Machine Learning (ML): it involves the usage of ML techniques such as CRF, SVM, Logistic Regression (LR) and etc. for extracting information from free texts.

However, to the best of our knowledge, there is no open NLP pipeline from which we can extract information related to the lung cancer tumor mutation status, tumor stage and PS, written in Spanish clinical narratives. Thus, the main objective of this Chapter is to discuss the design, development and the implementation of annotators, capable of detecting these clinical information from EHRs using UIMA framework. Furthermore, we present the annotation results extracted by means of running these annotators.

The rest of the Chapter is organized as follow. Firstly, Section 2.2 discusses the problem statement. Afterwards, Section 2.3 provides a comprehensive review of the literature on NLP. Then, Section 2.4 presents the solution for annotating tumor mutation status, tumor stage and PS concepts related to lung cancer domain along with the process of annotation output generation. Finally, Section 2.5 presents the results generated through means of validating 
Chapter 2. Lung Cancer Concept Annotation from Spanish Clinical Narratives 2.2. Problem Statement

these annotators on a large collection of EHRs and Section 2.6 explains the achievements gained.

\subsection{Problem Statement}

The NER process intrinsically relies on ontologies, taxonomies and controlled vocabularies such as Systematized Nomenclature of Medicine (SNOMED) [50] and Unified Medical Language System (UMLS) [51,52]. Even though that the translations of these vocabularies to different languages are available, they do not always provide the entire terminologies that are used in very specific domains (e.g., lung cancer).

In addition, several medical metrics are not covered or fully provided by the vocabularies. Furthermore, symbols such as "+" and "-", which are commonly being used with medical metrics (e.g., $E G F R+$ ) to determine their positivity or negativity, are not supported by them. Also, it is a common practice by physicians to use symbols such as ".", "-", "-", etc. for writing metrics (e.g., Stage I-A1). Such metrics are not supported by these vocabularies as well.

Despite of the fact that Spanish language has occupied the second position in the world ranking of number of native speakers with more than 572 million speakers [20], most of NLP systems have been developed to extract information from clinical texts written in English. Although few systems have been introduced for the detection of concepts from Spanish clinical texts, to the best of our knowledge, there is no open-source system from which we can extract information related to the tumor mutation status, tumor stage and the PS from EHRs.

\subsection{State of the Art}

The main objectives of this Section is to: (1) review the NLP preliminaries in Section 2.3.1 (2) describe the frameworks that can be used for developing 
Chapter 2. Lung Cancer Concept Annotation from Spanish Clinical Narratives 2.3. State of the Art

annotators in Section 2.3.2 and (3) examine the recent published research on extraction of information from free texts of EHRs in Section 2.3.3.

\subsubsection{NLP Preliminaries}

The typical NLP process serves to the purpose of analyzing free texts and extracting useful information from them. This process includes a pipeline of components (Figure 2.1), responsible for detecting sentences, tokens, Part of Speech $(\overline{\mathrm{PoS}})$, phrases, parse trees and entities such as people, locations, or in case of the health care, parts of body, drugs, diseases, etc. from free texts.

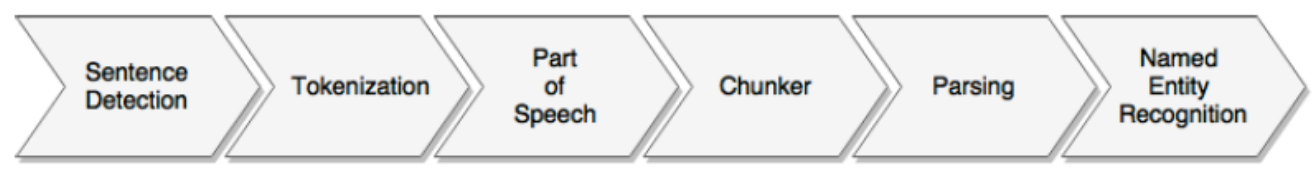

Figure 2.1: A typical NLP pipeline 2

\subsubsection{Frameworks for Building Annotators}

In this Section, we review the three of most popular frameworks introduced for design and development of annotators.

Firstly, the General Architecture for Text Engineering (GATE) framework [53,54] is a Java-based open-source software, which allows the users to develop and deploy NLP components for extraction of information from free texts. This framework is developed by the University of Sheffield in 2002.

Protégé 55 is another free and open-source framework and ontology editor introduced by the Stanford University for building intelligent systems. It can be adapted for building both simple and complex ontology-based applications. The output of Protégé can be integrated with rule-based systems or other problem solvers for constructing a wide range of intelligent systems.

UIMA [3, 24] is a software architecture, created by David Ferrucci and Adam Lally at IBM back in 2004. Since, we have used UIMA framework in 
Chapter 2. Lung Cancer Concept Annotation from Spanish Clinical Narratives 2.3. State of the Art

the design and development of our annotators, for the sake of completeness, we provide the detailed information about this software architecture.

UIMA is used for developing Unstructured Information Management (UIM) applications, which can analyze large amounts of unstructured data (texts, images, voice records, etc.). To be able to develop UIM applications, UIMA provides a set of frameworks to interact with using $\mathrm{C}++$ and Java. These frameworks offer an Application Programming Interface (API) for enabling the connection of user applications to the UIMA engine. In addition to the frameworks, UIMA also provides an infrastructure containing servers and tools for scaling up the UIM applications and the core components such as annotators and type systems that can be developed in the user work space. For quick hands-on what UIMA offers, see Figure 2.2 .

When an application is developed on top of UIMA framework, the objects definition is done by the developer but the execution is done by the UIMA core engine. Based on the developer's definition, UIMA creates a set of annotations from source documents to be analyzed by the end user. These annotations are stored in a data structure, known as Common Analysis Structure (CAS). A CAS contains data to be annotated (the raw texts in the case of analyzing textual documents), and indexes with the different annotations once the document has been processed.

The developer must create several components to define the objects that will be annotated. An example of such object can be the tokens defined in a sentence. These components to be developed are:

1. Annotator: is a basic component type, which is implemented as a class in the chosen programming language and determines what has to be done. It contains the core analysis algorithm and a "process" method that is called upon its execution.

2. Type System: is a schema that defines the properties and types of objects to be annotated. Each annotator has a type system which may have as many items as desired. These items can be of native types (integers, floats, strings, etc.) or data structure types (arrays and lists). An example of these items is the type of token (if it is a punctuation symbol, a word, etc.) 
Chapter 2. Lung Cancer Concept Annotation from Spanish Clinical Narratives

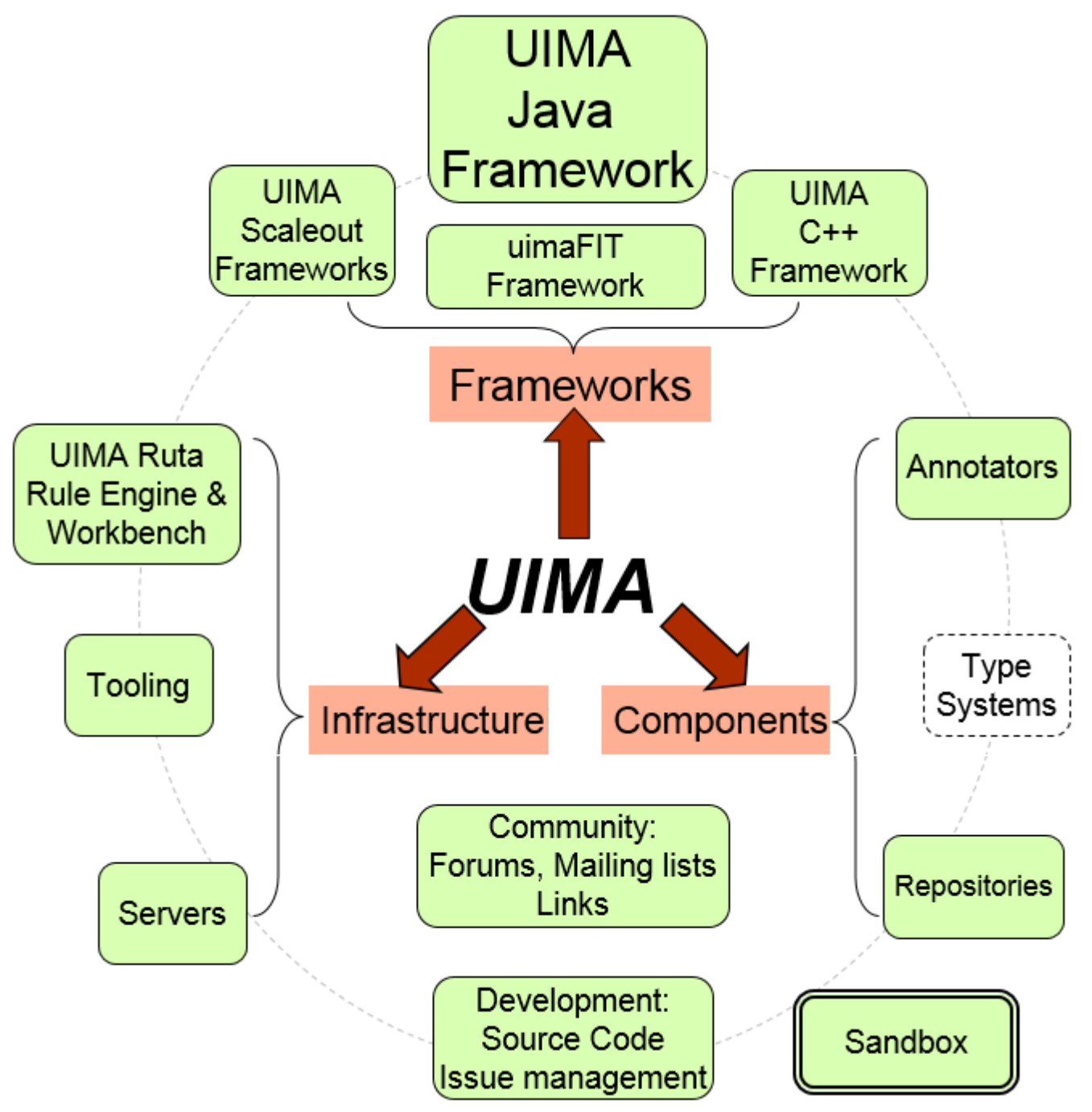

Figure 2.2: UIMA frameworks, infrastructure and components 3

3. Collection Processing Engine (CPE) Descriptor: is typically specified in an XML file that defines which are the annotators to be executed, the type systems to be used and the expected inputs and outputs. A CPE descriptor can be a simple descriptor in which only one annotator can be executed or an aggregated one in which several annotators can be executed in a single-flow pipeline or a parallel one. 
Chapter 2. Lung Cancer Concept Annotation from Spanish Clinical Narratives 2.3. State of the Art

Once the programmer has defined what and how to annotate the objects represented, the CPE Factory gathers the source documents, creates Analysis Engines (AES) to run the descriptors, and creates the corresponding CASes. Figure 2.3 presents an overview of the UIMA architecture.

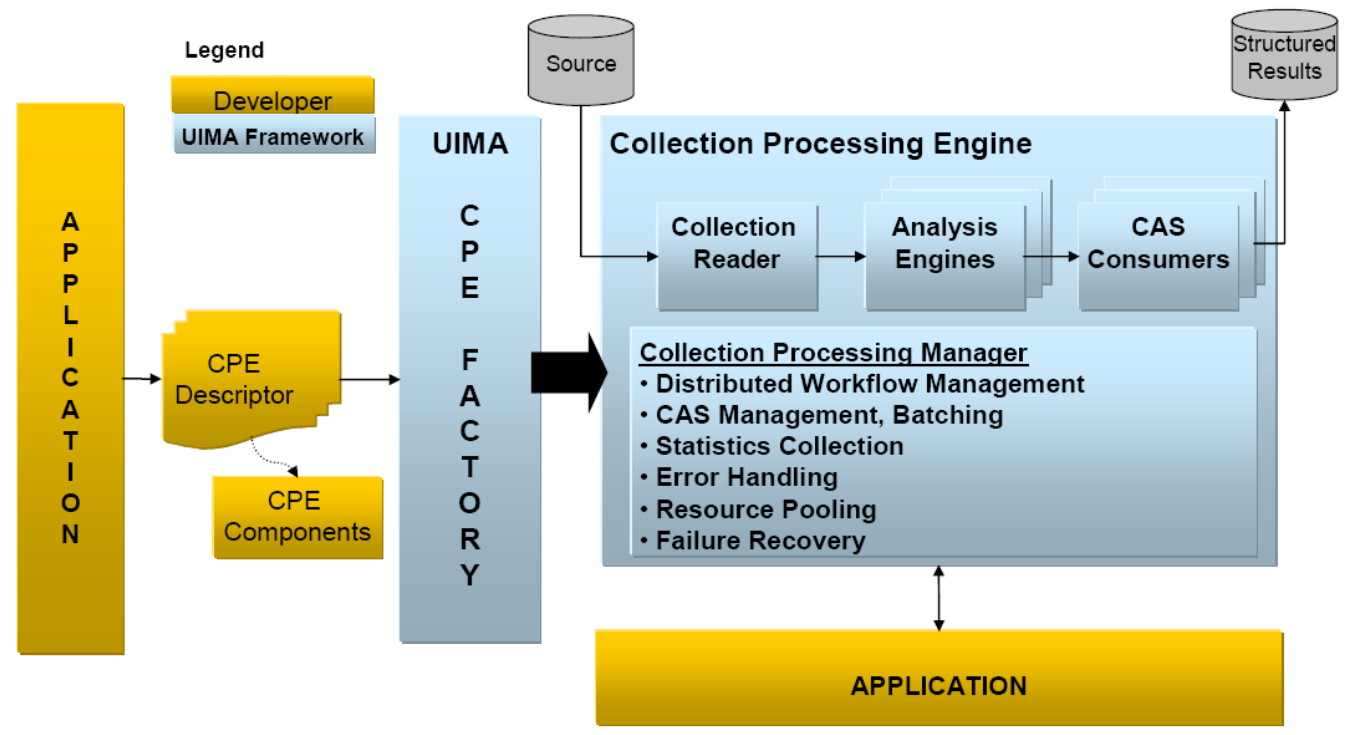

Figure 2.3: UIMA architecture 4

\subsubsection{Extraction of Information from Free Texts of EHRs}

The rapid adaption of EHRs with the parallel growth of electronic narrative data have created a great interest of harvesting clinical information and knowledge from free texts. The extraction of information from EHRs supports both clinical and transitional research.

Much of the clinical data are stored in an unstructured format within EHRs, i.e., written in textual form [56]. Although free texts facilitate more complete and expressive descriptions of clinical information, which would have not been possible through structured format, they can not be efficiently used for the tasks of searching, decision support, summarization, and statistical analysis. To decrease the error rate and improve the quality control, structured data is required; this is where NLP comes to the picture. 
Chapter 2. Lung Cancer Concept Annotation from Spanish Clinical Narratives 2.3. State of the Art

NLP research focuses on developing computational models for understanding the language used by human beings [57]. An NLP system can contain syntactic processing modules (e.g., tokenization, sentence detection, PoS tagging, chunking, etc.) and/or semantic processing modules (e.g. NER, relation identification, etc.). However, an NLP system with semantic processing modules for extraction of predefined types from free texts is known as an IE application [58,59]. In general, IE is recognized as a specialized area under the empirical NLP.

According to the International Medical Informatics Association (IMIA) yearbook [60,61], medical NLP is defined as NLP to clinical texts or aimed at a clinical outcome. This encompasses NLP applied to the free texts in EHRs that is the case of information extraction for decision support or clinical research.

Several reviews have been conducted on medical NLP research such as from 1995 to 2008 in [57], during 1996 in [62] and from 2009 to 2016 in [49]. One review was also conducted with the focus on medical NLP applications in languages other than English from 2017 to 2018 in [63]. Other reviews focused on NLP in specific domain such as potential application of NLP in cancer-case identification, staging and outcomes quantification in [64], and NLP methods and tools, which support practical applications in radiology in [19].

In this Section, we survey studies conducted from 1994 to 2020 and seek to provide insight on major developments in medical NLP domain for English and Spanish languages.

\section{Review Method and Selection Criteria}

Conduction of a comprehensive survey on medical NLP is not a straightforward task as related studies are spread over the literature of several fields of medical informatics, NLP and computer science. Furthermore, as the language addressed in the studies is not always mentioned in the title or abstract of the articles, building search quires with high specificity and sensitivity is very difficult. 
Chapter 2. Lung Cancer Concept Annotation from Spanish Clinical Narratives 2.3. State of the Art

To approximate the NLP publication trends in the medical field for all languages, English and Spanish, we used three very broad PubMed queries 65] for articles published from 1994 to 2020. It is worth mentioning that the number of publication acquired from 2020 covers only the first six of this year. As some publications on NLP research in medical field do not appear in PubMed or some publications referenced in PubMed may be missed by our queries, we present the result of these queries as an imperfect proxy to estimate the scale of NLP research in this specific field.

Our first PubMed query of "Medical Natural Language Processing" aimed to approximate the NLP publication trends in the medical domain for all the languages. This query returned 3,773 results. Figure 2.4 shows the evolution of the number of NLP publications, which are retrieved from the query. We can see that the amount of publications was very few (only 19 publications) in 1994. Afterwards, this amount had small fluctuations until 2004 and then increased exponentially with some moderate fluctuations to 2019. In 2019, this number was at its pick with 463 publications.

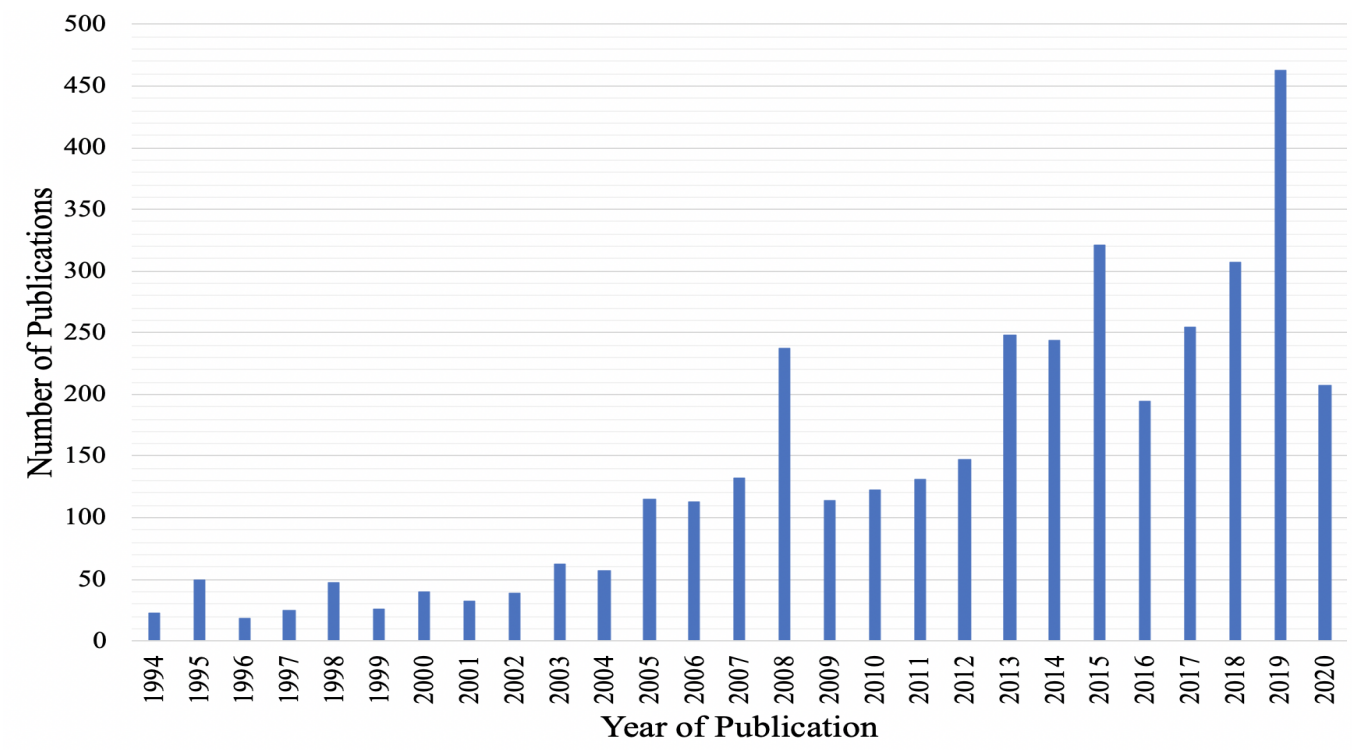

Figure 2.4: The distribution of NLP publications in medical domain from 1994 through 2020 
Chapter 2. Lung Cancer Concept Annotation from Spanish Clinical Narratives 2.3. State of the Art

While the second PubMed query of "Medical Natural Language Processing in English" returned 3,752 results, the third PubMed query of "Medical Natural Language Processing in Spanish", resulted in only 39 articles.

Figure 2.5 compares the number of medical NLP articles published in English and Spanish. As one can see, the number of English related articles have grown tremendously from 1994 to 2019 whereas the Spanish related publications are very few and do not show a significant evolution. NLP in medical domain has seen its largest number of publication for English in 2019 with 459 articles and its lowest number of publications in 1996 with 19 articles. However, the Spanish related articles in this field were at its pick in 2015 and 2019 with 7 publications and also for many years no Spanish related articles were published. Therefore, the under utilization of NLP in medical domain for Spanish language requires more attention by the researchers.

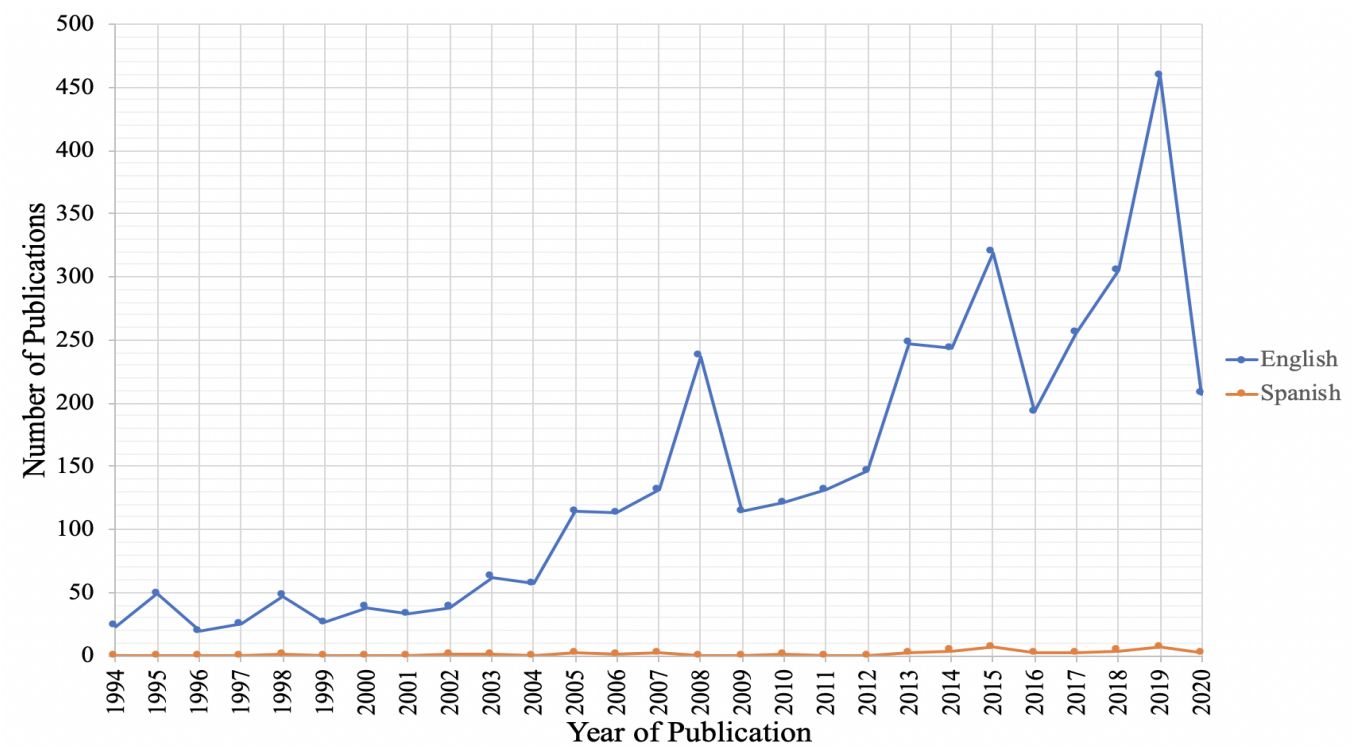

Figure 2.5: Comparison of the number of NLP publications in medical domain for English versus Spanish from 1994 through 2020

This state of the art does not provide a systematic review of medical NLP literature but rather aims to present a selection of studies to cover a representative number of NLP methods and systems developed for medical 
Chapter 2. Lung Cancer Concept Annotation from Spanish Clinical Narratives 2.3. State of the Art

knowledge extraction from EHRs, written in English and Spanish. We browsed the results of the broad queries in PubMed, Medical Literature Analysis and Retrieval System Online (MEDLINE) or MEDLARS Online [66], Association for Computational Lingustics (ACL) anthology [67], Association for Computing Machinery (ACM) digital library [68], Springer [69], conference proceedings and journals. We also leveraged our own literature knowledge in the medical NLP in languages of English and Spanish.

Our selection criteria was based on the IMIA definition of medical NLP 60, 61]. For example, the broad quires used in ACM resulted in a number of publications on general free texts, not clinical texts processing, which we excluded. Furthermore, with the great volume of the articles published in this area, we prioritized the inclusion of studies about medical concept extraction than general concepts such as token, PoS tags, negations, etc. We also added relevant publications and websites referenced in the papers. In total, 63 publications fulfilled these criteria such that 58 of them were selected for English and 5 of them were chosen for Spanish.

The following Sections review NLP systems developed for extraction of information from English clinical texts, English oncological texts and Spanish clinical texts.

\section{Medical NLP on English Clinical Texts}

The growing interest in the development of NLP methods to be applied in the health care domain and the increasing use of EHRs have created a significant development of so called Medical Language Processing (MLP) systems. These systems are able to analyze free texts of different types of clinical reports generated (e.g., radiology, mammography, pathology, discharge summaries, biopsy, etc.). In here, we extensively review the MLP systems developed and used for EHRs, written in English.

In 1994, a research effort at LDS hospital of Salt Lake city, USA focused on building a Natural Language Understanding System (NLUS), capable of analyzing chest $\mathrm{x}$-ray reports and storing the extracted clinical data into a medical database [70]. This effort resulted in a natural language parser called Symbolic Text Processor (SymText) 71] in 1995. SymText combines a 
Chapter 2. Lung Cancer Concept Annotation from Spanish Clinical Narratives 2.3. State of the Art

syntactic parser using augmented transition networks and transformational grammes with a model of semantics based on Bayesian Networks (BNs) statistical formalism.

Later in 1998, SymText was used to extract relevant clinical information from the radiology (Ventilation / Perfusion lung scan) reports [72]. In addition, in 2003, it was evaluated on the chest radiograph reports in order to establish whether it can identify the mention of Central Venous Catheter (CVC) from clinical texts 73$]$.

In 1994, MENELAS [74] was also presented as an NLP system, capable of extracting information from patients discharge summaries. This system supports processing of clinical texts written in French, English and Dutch. It encodes the free texts of discharge summaries into both an internal representation and International Classification of Diseases-Ninth Revision-Clinical Modification (ICD-9-CM) nomenclature codes.

In context of 1995, Medical Language Extraction and Encoding System (MedLEE) [75] was developed by Carol Friedman. MedLEE is a rule-based NLP system, which extracts, encodes and structures clinical information appeared in the patients textual reports written in English. It was originated to work on chest radiological reports for decision support applications and then extended to process mammography reports and discharge summaries [76].

In 1999, the relevance of NLP in medicine was discussed by the same authors of MedLEE in [77], presented a use case of analyzing the severity of pneumonia cases in discharge summaries [78. In 2000, MedLEE was improved by introducing the vocabulary development and automated encoding of textual clinical information into ICD-9, SNOMED and the UMLS codes [79]. It was also extended to process electrocardiography, echocardiography, pathology and all types of radiology reports.

The performance of MedLEE was then evaluated in [80 using different lexicons (LUMLS, M+UMLS and M-CUR) in 2001. In the included studies, MedLEE has been used to detect suicide or suicidal attempts [81], to recognize Pharmacovigilance-related adverse events [82] and to identify overuse medications patterns [83]. 
Chapter 2. Lung Cancer Concept Annotation from Spanish Clinical Narratives 2.3. State of the Art

In 2001, one of the most popular applications of NLP for analyzing biomedical texts was introduced as MetaMap [84,85]. MetaMap is a software, capable of mapping medical terms found in biomedical texts to knowledge sources like UMLS and Medical Subject Headings (MeSH) 86, 87]. It is designed to parse texts, generate variants, analyze acronyms and abbreviations, and find synonyms. It is introduced as a generic set of algorithms not only specific for biomedical domain but also extensible to other domains with appropriate knowledge sources.

MetaMap has been used for detection of pneumonia and influenza death cases from death certificates [88], extraction of phenotype information 89 91], assessment of emergency department use 92, 93], extraction of patientsrelated attributes [94], fragment recognition in clinical documents [95] and drug-disease treatment relationships [96].

In 2002, a tool for analyzing clinical texts using BNs was introduced in [97], known as MPLUS (M+). BNs of $\mathrm{M}+$ have similar structure to semantic networks by implementing directed acyclic graphs with nodes representing words and concept types, and links representing relations between those types.

In 2003, KnowledgeMap Concept Indexer (KMCI) [98, 99] was presented as an NLP system, which identifies biomedical concepts from medical educational texts and maps them to the UMLS concept. KMCI employs PoS information to generate a shallow sentence parse, and implements variant generation and normalization using the SPECIALIST Lexicon and related tools.

Clinical Text Analysis and Knowledge Extraction System (cTAKES) 100 , 101 is also one the most popular applications of NLP, which uses rulebased and machine learning techniques to process textual information. The development of cTAKES started at Mayo clinic, USA in 2010. cTAKES has been included in the Apache open-source catalog and is built using Apache UIMA framework and Apache OpenNLP toolkit [102].

The NLP pipeline of cTAKES is composed of the sentence boundary detector, tokenizer, normalizer, PoS tagger, and NER annotators. For implementing NER process, this system uses vocabularies such as UMLS. cTAKES has been 
Chapter 2. Lung Cancer Concept Annotation from Spanish Clinical Narratives 2.3. State of the Art

adapted for identification of the patient's smoking status [103 108], medications, risk factors and co-morbidities, genome-wide association studies [104], adverse drug events extraction [109], risk stratification [110] detection of patient phenotype cohorts [111], temporal relation discovery [112] and extraction of the patient's deep phenotype information [113].

The gene/protein name recognition system, known as BioTagger-GM [114] was adapted in 2011 to MedTagger [115, 116] for medical concept mention detection from clinical texts. MedTagger is used for indexing based on dictionaries and extracting information based on patterns and machine learning techniques. In 2013, MedTagger was integrated with cTAKES for medical concepts recognition and normalization based on CRF and a greedy dictionary lookup algorithm, respectively [117.

The Medication Extraction (MedEx) system [118] was developed to accurately extract medication names and signatures from clinical narratives in 2010. MedEx was initially developed using discharge summaries and implemented a sequential semantic tagger and a Chart parser to map medication texts into a structured representation.

In 2010, the open-source Automated Retrieval Console (ARC) 119 121] was introduced to combine the NLP pipelines with machine learning techniques for processing unstructured data to structured data such as UMLS and SNOMED codes. In 2011, ARC was used to extract information from cancer related pathology and imaging reports 122$]$.

In the year 2012, Health Information Text Extraction (HITEx) 123, 124 was introduced as an open-source NLP software application. HITEx makes use of Gate framework to analyze common problems such as extraction of principal diagnosis and comorbidities associated with a hospitalization and detection of smoking status of patients from clinical narratives.

The 11 modules of GATE framework used by HITEx are: (1) section splitter, (2) section filter; (3) sentence splitter; (4) sentence tokenizer; (5) PoS tagger; (6) noun phrase finder; (7) UMLS concept mapper; (8) negation finder; (9) N-gram tool; (10) classifier; and (11) regular expression-based concept finder. 
Chapter 2. Lung Cancer Concept Annotation from Spanish Clinical Narratives 2.3. State of the Art

In addition, an approach to extract information from clinical texts was introduced in [125 in the same year. Using CRF and keyword matching with terminology-based disambiguation, the information are extracted from clinical plain texts and then, integrated into a data warehouse.

Automatic knowledge acquisition of diagnostic and therapeutic procedures from clinical texts is analyzed in [126] in 2013. The authors of this work built a new system by reusing open-source NLP tools of OpenNLP, Stanford parsers [127], SemRep [128] and UMLS NormalizingString service.

In 2014, several clinical NLP systems were also described under a unified architecture view in [129]. Two main components were combined in this architecture: (1) background knowledge, which includes biomedical knowledge resources; and (2) a framework, which integrates NLP tools to process clinical texts.

In the same year, the Medication Extraction and Normalization (MedXN) system [130,131] was developed to extract comprehensive medication information from clinical texts. MedXN also normalizes the extracted information to the most appropriate RxNorm Concept Unique Identifier ( $\mathrm{RxCUI}$ ).

In 2018, Clinical Language Annotation, Modeling, and Processing (CLAMP) toolkit 132, 133 was developed as a clinical NLP software using UIMA framework. CLAMP enables the recognition of clinical information in patients reports. In addition, it provides a graphical user interface, which helps the users to build customized NLP pipeline for different applications.

\section{Medical NLP on English Oncological Texts}

There exist also systems that envision the power of NLP to benefit oncological domain, applying algorithms to extract knowledge from EHRs, provided in English. Some of these systems have been described in 134 138].

In 2006, a system was developed to extract pathologic $\mathrm{T}$ and $\mathrm{N}$ codes of TNM system related to lung cancer domain from histology reports [134. This system makes use of NLP techniques to transform report texts, including the detection of UMLS terms and identification of the negated findings. The 
Chapter 2. Lung Cancer Concept Annotation from Spanish Clinical Narratives 2.3. State of the Art

transformed report is then classified using SVMs. For T classification, the presented system gained macro-average F1 score of 0.57 and micro-average of F1 score of 0.61 . In addition, for $\mathrm{N}$ classification it obtained the macro-average F1 score of 0.70 and micro-average of F1 score of 0.78 .

In 2007, the Case Finding Engine (CaFE) tool [136] was developed to scan free texts of medical documents for identifying and highlighting the concepts relevant to cancer. CaFE includes 800 SNOMED codes and a custom-made list of approximately 2,500 terms and phrases that were created by reading pathology reports and by input from a cancer registry team. Using SNOMED codes and custom-made list, CaFE searches for the concepts in clinical texts and highlight the identified ones.

In the context of 2009 and as an extension to Medical Text Analysis System (MedTAS) [139], IBM developed the pathology version of it, known as MedTAS/P 135]. The aim of MedTAS/P is to map the concepts from pathology reports into the ones represented by the Cancer Disease Knowledge Representation Model (CDKRM) using rule-based and machine learning techniques. For this purpose, MedTAS/P extracts cancer-specific characteristics such as grade, stage, size, margin, date, and tumor blocks from the pathology reports and discover the relationships between them.

Furthermore, the cancer Tissue Information Extraction System (caTIES) [137, 140] is an open-source text mining tool, developed in the year 2010 with the aim to automatically extract concepts such as tumor grade, stage, body parts, procedures and diseases from free texts of pathology reports. These concepts are then stored as structured data in a database, which facilitates advanced query and analysis.

In 2014, the validity of an NLP program to identify patients suffering from prostate cancer after biopsy and to retrieve pathologic information from their EHRs was assessed in 138.

\section{Medical NLP on Spanish Clinical Texts}

For analyzing EHRs written in Spanish, only a few systems have been presented in $2,21-23$. 
Chapter 2. Lung Cancer Concept Annotation from Spanish Clinical Narratives 2.4. Solution

In 2014, Text and Images Data Analyzer (TIDA) 21, 141] was introduced as an architecture, containing a semantic search engine, which was built upon Apache UIMA framework and using Apache OpenNLP toolkit. TIDA is capable of performing three tasks: (1) processing clinical notes and radiological information to identify medical terms and negations; (2) processing images and other medias to detect patterns of abnormality and key factors required by the clinicians to complete a patient's study; and (3) extracting knowledge from structured information sources using big data analytics techniques. In 2016, TIDA was renamed to Human Health Analytics ( was used to extract stroke scale from clinical narratives.

Further in 2018, Clinical Knowledge Extraction System (C-liKES) 22] was introduced as a text mining system, which was developed on top of Apache UIMA framework and based on the legacy system of H2A. C-liKES ingests clinical information of EHRs and yield a structured output that can support complex quires and be used for applying in-depth analytics.

In the same year, SAVANA 23, 142 was also presented as an NLP platform for clinical decision support based on real-time dynamic extraction of information written in EHRs corpora. It performs statistical analysis of patients seen in the platform and provides the relevant results to end users.

Finally, based on the comprehensive review of the literature and to the best of our knowledge, no system has been introduced for identification of tumor mutation status, tumor stage and PS related to lung cancer domain from Spanish clinical texts. Therefore, in the line of this research, we propose development of annotators, capable of recognizing these specific concepts.

\subsection{Solution}

We have developed a set of semantic rule-based NLP modules, named Annotators using Apache UIMA framework. These annotators were developed to identify NEs from clinical narratives. They contain regular expressions using which, they can search for specific patterns through clinical texts. 
Chapter 2. Lung Cancer Concept Annotation from Spanish Clinical Narratives 2.4. Solution

The Pseudocode of algorithm implemented by these annotators is provided in the Listing 2.1. The algorithm defines the search pattern using regular expressions and accepts the plain texts of EHRs as input. Then, for each individual sentence in the texts, the algorithm checks if the search pattern can be matched with the tokens. Once, a matched token is found, the algorithm assigns the semantical meaning corresponding to the token and produces the output by adding annotation to the token indexes.

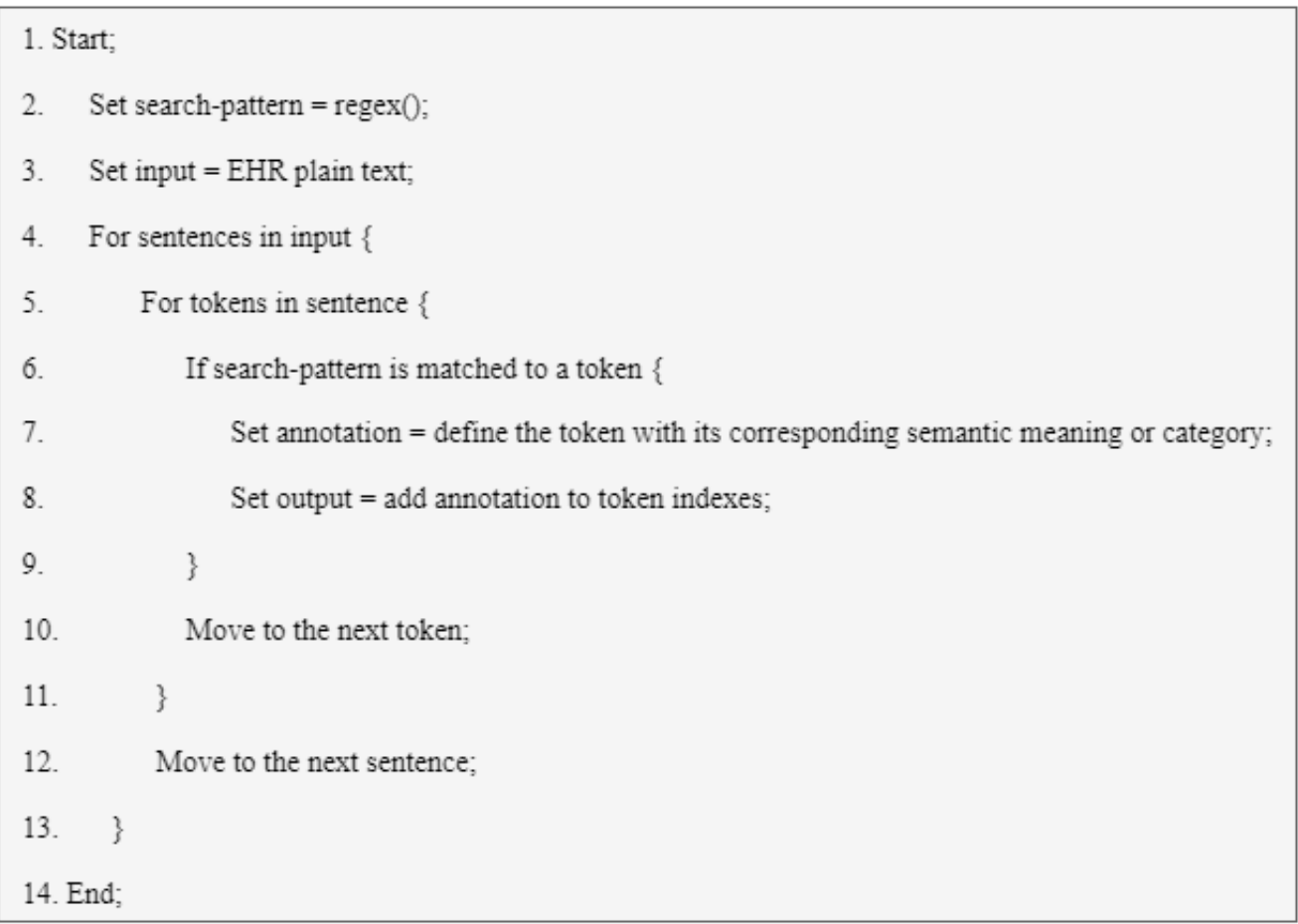

Listing 2.1: Pseudocode of algorithm for rule-based annotators

To process EHRs using these developed annotators, we have implemented them under a single NLP pipeline. Once, the pipeline is executed, the output of annotations will be generated. These outputs are formatted as a set of XMI files and are also inserted into a relational database from which QA process can be followed. 
Chapter 2. Lung Cancer Concept Annotation from Spanish Clinical Narratives 2.4. Solution

The details of the annotators developed for recognition of tumor mutation status, tumor stage and performance status concepts are provided in the Sections 2.4.1, 2.4.2 2.4.3, respectively. In addition, the process of annotations output generation is discussed in Section 2.4.4.

\subsubsection{Tumor Mutation Status Recognition}

Clinicians make use of EGFR, ALK and ROS1 metrics for mentioning tumor mutation status in clinical narratives. However, in case of EGFR, they can provide more detailed information about mutation status related to exon (18-21), type of exon (deletion or insertion) and mutation point (G719X, T790 M, L858R, L861Q).

For determining the positivity or negativity of mutation metrics, clinicians do not follow any standard systems. For example, in case of "EGFR positive", they can write: "EGFR: positive", "EGFR+", "Has detected with mutation in EGFR", "Presence of mutation in EGFR", "With insertion mutation in Exon 19", "EGFR mutated", etc. Therefore, the need for annotators to detect tumor mutation status from clinical texts, comes to the picture. For this purpose, three annotators, named EGFR Annotator, ALK Annotator and ROS1 Annotator were developed.

The EGFR Annotator is capable of detecting the tumor mutation status, exon, type of exon and the mutation point from clinical texts by incorporating four internal annotators that were developed for this purpose. Whereas the ALK and ROS1 annotators can only find the concepts that are related to the mutation status.

For example, in the following clinical text: "EGFR + (del exon 19), no se detecta traslocación de ALK y ROS1 no traslocado." while EGFR mutation status is positive in exon 19, no translocation is detected for ALK and ROS1. Once, we process this clinical text using the developed annotators of tumor mutation status on UIMA CAS Visual Debugger (CVD), this information is extracted from the text (Figure 2.6). The CVD output representation is largely divided into two sections:

1. Analysis Result: is composed of two subsections: 
Chapter 2. Lung Cancer Concept Annotation from Spanish Clinical Narratives 2.4. Solution

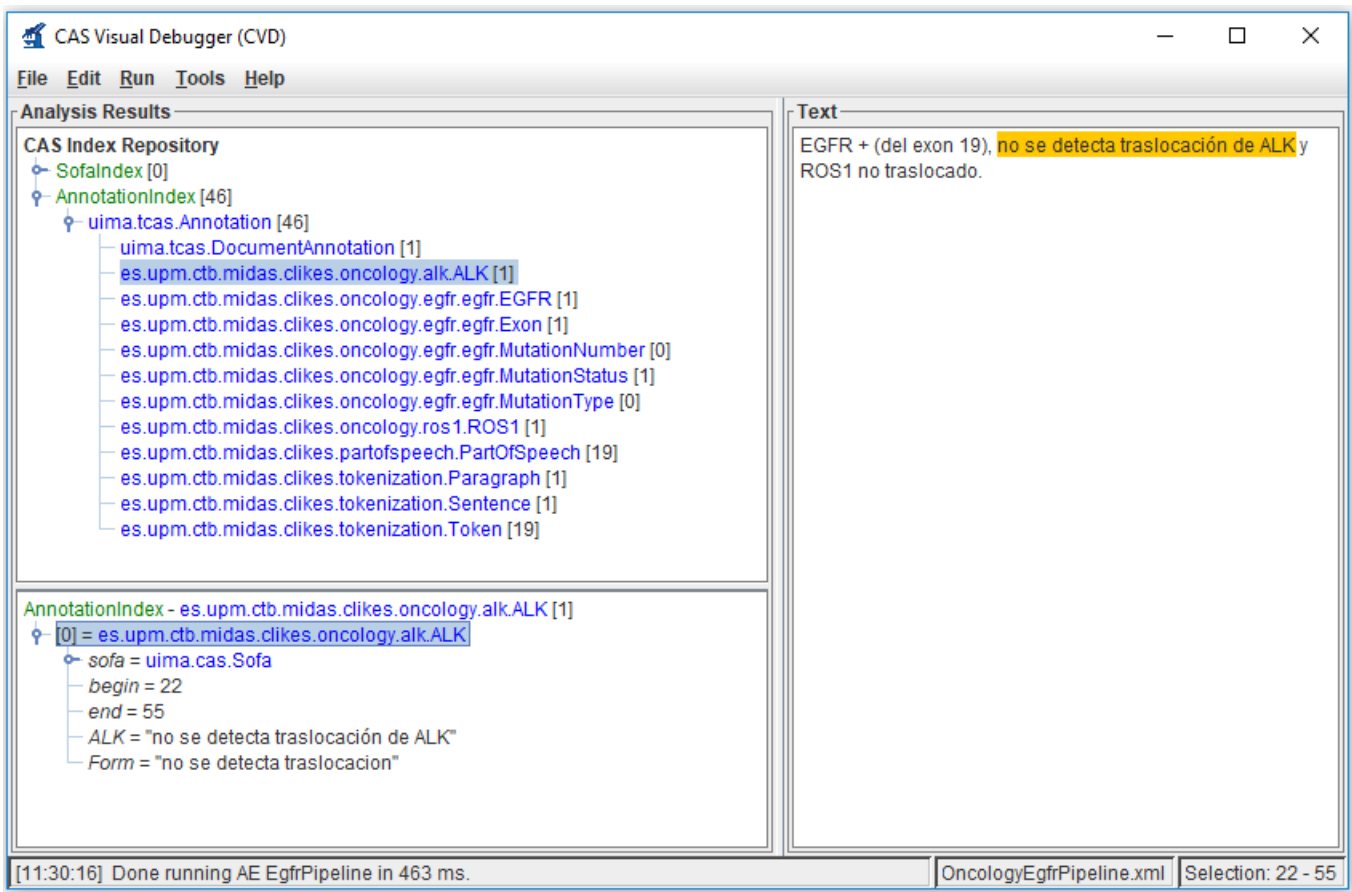

Figure 2.6: The annotation results of EGFR, ALK and ROS1 annotators on CVD

- Upper division: contains the CAS Index Repository in which the AnnotationIndex presents the list of annotators executed for processing the text. To see the annotation results of a specific annotator, the user should click on the designated one.

- Lower division: includes the AnnotationIndex. When the user clicks on the index of an annotation (in the Figure 2.6, ALK) the information such as begin, end and semantic categories of the found NE will be represented.

2. Text: accepts clinical texts as input from user, which is in here "EGFR + (del exon 19), no se detecta traslocación de ALK y ROS1 no traslocado.". The input text will be highlighted corresponding to the begin and end of annotation provided in the AnnotationIndex of the lower division. The highlighted text in the Figure 2.6 is "no se detecta traslocación de ALK". 
Chapter 2. Lung Cancer Concept Annotation from Spanish Clinical Narratives 2.4. Solution

\subsubsection{Tumor Stage Recognition}

Stage Grouping and TNM are the two main standard cancer staging systems, introduced by AJCC manual [5,45]. The lung cancer stage classification, which is provided by the International Association for the Study of Lung Cancer (IASLC), is based on the advanced statistical analysis of international database with more than 100,000 patients. This analysis specifically addressed the stage groups, T, N and M components (Figure 2.7).

It is notable that for TNM system, the three attributes, i.e., T, N and $\mathrm{M}$ can be modulated by prefixes (e.g., pT1aNOMO). Such prefixes are "c" (clinical), "p" (pathologic), "yc" or "yp" (post therapy), "r" (retreatment) and "a" (autopsy). Clinicians normally use symbols such as ".", "., " “", "()", etc. combined with TNM and stages metrics in clinical texts. For example, in case of "Stage IA1", they can write: "Stage I-A1", "Stage I.A1", "Stage $I_{-} A_{-} 1$ ", "Stage I(A1)", etc.

\begin{tabular}{|l|c||c|c|c|c|}
\hline T/M & Subcategory & N0 & N1 & N2 & N3 \\
\hline \hline \multirow{2}{*}{ T1 } & T1a & IA1 & IIB & IIIA & IIIB \\
\cline { 2 - 5 } & T1b & IA2 & IIB & IIIA & IIIB \\
\cline { 2 - 5 } & T1c & IA3 & IIB & IIIA & IIIB \\
\hline \multirow{2}{*}{ T2 } & T2a & IB & IIB & IIIA & IIIB \\
\cline { 2 - 6 } & T2b & IIA & IIB & IIIA & IIIB \\
\hline T3 & T3 & IIB & IIIA & IIIB & IIIC \\
\hline T4 & T4 & IIIA & IIIA & IIIB & IIIC \\
\hline \multirow{2}{*}{ M1 } & M1a & IVA & IVA & IVA & IVA \\
\cline { 2 - 6 } & M1b & IVA & IVA & IVA & IVA \\
\cline { 2 - 6 } & M1c & IVB & IVB & IVB & IVB \\
\hline
\end{tabular}

Figure 2.7: AJCC 8th edition - lung cancer stage grouping and TNM system 5

Using 8th edition of AJCC manual, we have developed two pattern-based extraction annotators, named Stage Annotator and TNM Annotator for finding the concepts of cancer stage and TNM appeared in the clinical narratives, respectively. For example, the clinical text "Adenocarcinoma de pulmón, 
Chapter 2. Lung Cancer Concept Annotation from Spanish Clinical Narratives 2.4. Solution

pT1aN0M0 (micronódulos pulmonares bilaterales, linfangitis carcinomatosa, derrame pleural), estádio $I_{-}$A1.", explains that the patient is having lung cancer (Adenocarcinoma de pulmón) with pT1aN0M0 value for TNM and stage of IA1. By processing this text using the Stage and TNM annotators, the information related to these two metrics have been annotated (Figure 2.8).

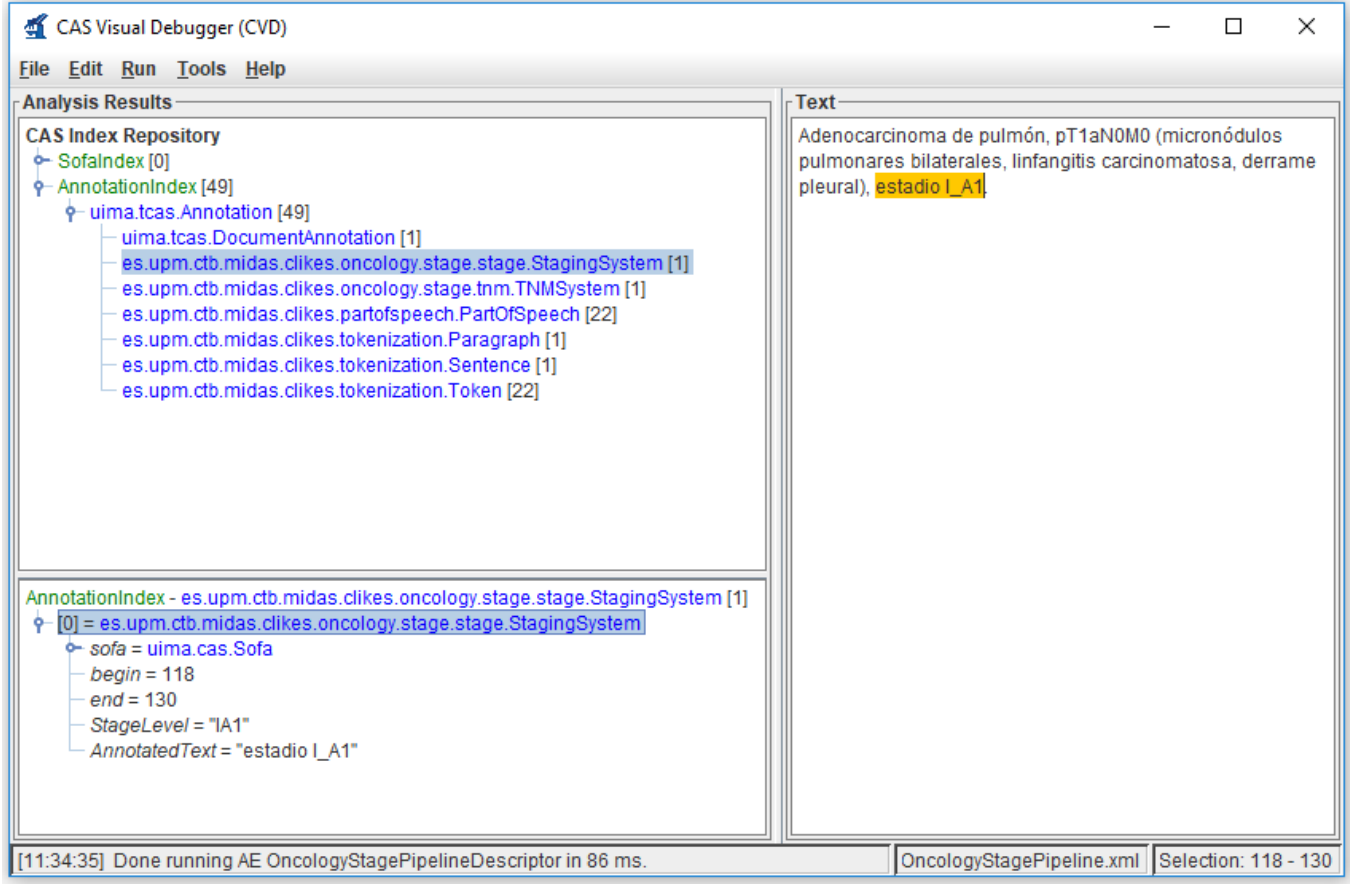

Figure 2.8: The annotation results of Stage and TNM annotators on CVD

\subsubsection{Performance Status Recognition}

PS scale is mentioned using ECOG and Karnofsky measures in clinical narratives. The ECOG measure ranges from 0 to 5 , where 0 is the most ideal case for carrying on all pre-disease performance without any restrictions. On the other hand, the Karnofsky measure ranges from $0 \%$ to $100 \%$, where $100 \%$ is the most ideal case.

In clinical texts, ECOG and Karnfosky scales can appear with symbols such as ".", "_", “-", "()", etc. For example, "ECOG 0" can be written as: 
Chapter 2. Lung Cancer Concept Annotation from Spanish Clinical Narratives 2.4. Solution

"ECOG_PS: 0", "ECOG-0", "ECOG is measured with 0", "ECOG (0)", etc.

Hence, to annotate concepts related to ECOG and Karnofsky scales, two annotators, named ECOG Annotator and Karnofsky Annotator were developed, respectively.

For example, "ECOG-PS 0. Regular estado general. Disnea de reposo/minimos esfuerzos. Karnofsky: 100\%.", indicates that the patient's PS is measured with ECOG of 0 and Karnofsky of $100 \%$. The results of the annotation processes implemented by the ECOG and Karnofsky annotators on this clinical text are shown in Figure 2.9.

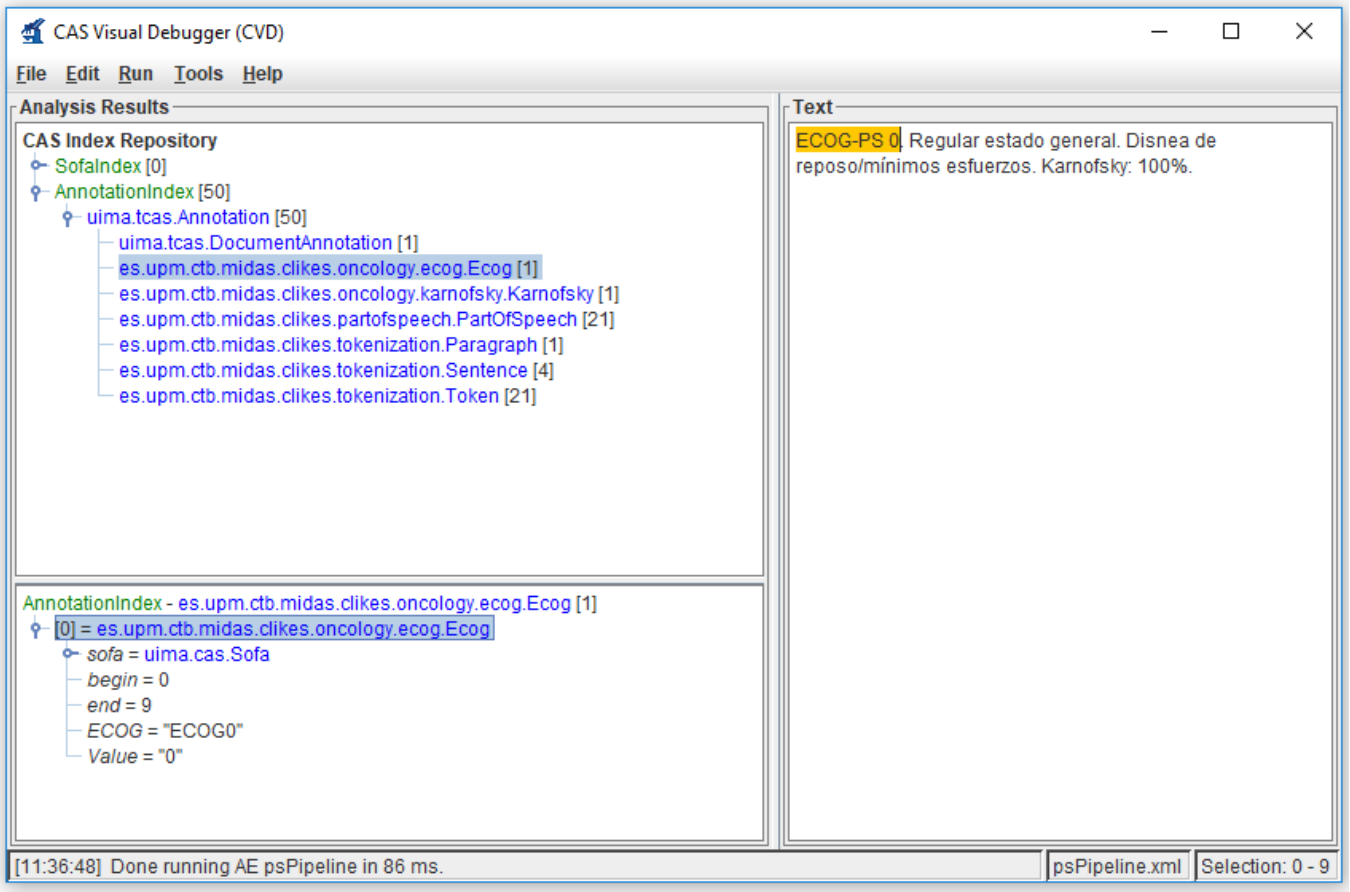

Figure 2.9: The annotation results of ECOG and Karnofsky annotators on CVD

\subsubsection{The Process of Annotations Output Generation}

For generating outcomes, an execution flow (Figure 2.10) is followed by using a main process, called Processing Engine. This process accepts plain text files 
Chapter 2. Lung Cancer Concept Annotation from Spanish Clinical Narratives 2.4. Solution

as input. Example of plain text documents are clinical reports, clinical notes, radiology reports, and any kind of medical textual documents generated.

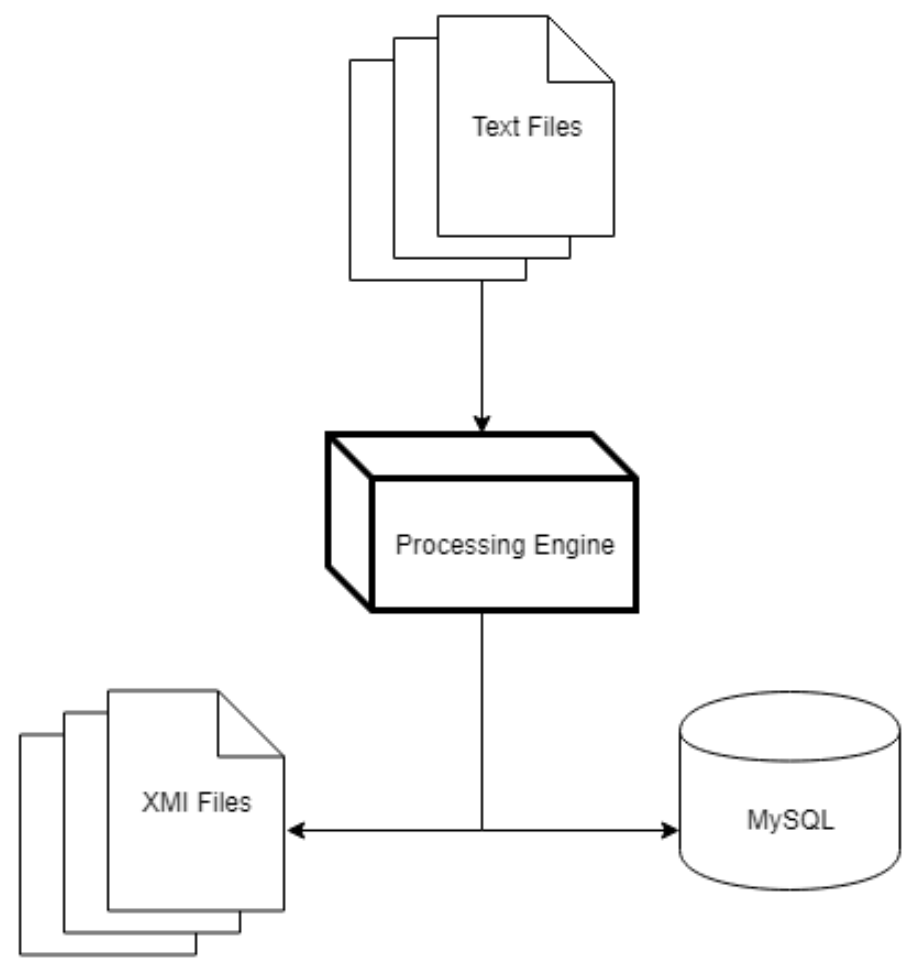

Figure 2.10: Processing engine architecture

The input is passed to the Processing Engine, which contains the pipeline of developed annotators. When the Processing Engine is executed, two resources are generated as output:

1. XMI: UIMA annotators process plain text documents and generate one XMI file for each of them. These files encompass all the existing annotations i.e., they contain structured data of the relevant unstructured data in the EHR. Figure 2.11 presents the results of annotations, stored in an XMI file, using UIMA annotation viewer. The UIMA annotation viewer is divided into three sections:

- Upper left division: contains plain clinical texts. Highlighted tokens correspond to the annotated concepts, which are in here 
Chapter 2. Lung Cancer Concept Annotation from Spanish Clinical Narratives 2.4. Solution

"ECOG 3", "estadio IV", "EGFR", "no mutatdo" and "ALK no traslocado";

- Annotation Type: provides the list of annotation tags from which the user can select the annotation results to be highlighted in the upperleft division section. In here such tags are ALK, ECOG, EGFR, and etc.

- Click In Text to See Annotation detail: by clicking on the highlighted tokens in the upper left division, the user can see the details of annotated concepts in this section. Such details are the begin, end and semantic categories of the concept.

2. Structured relational database: it is a MySQL database, which contains the information of annotations. The database allows to perform analysis on the structured data with more flexibility than XMI files.

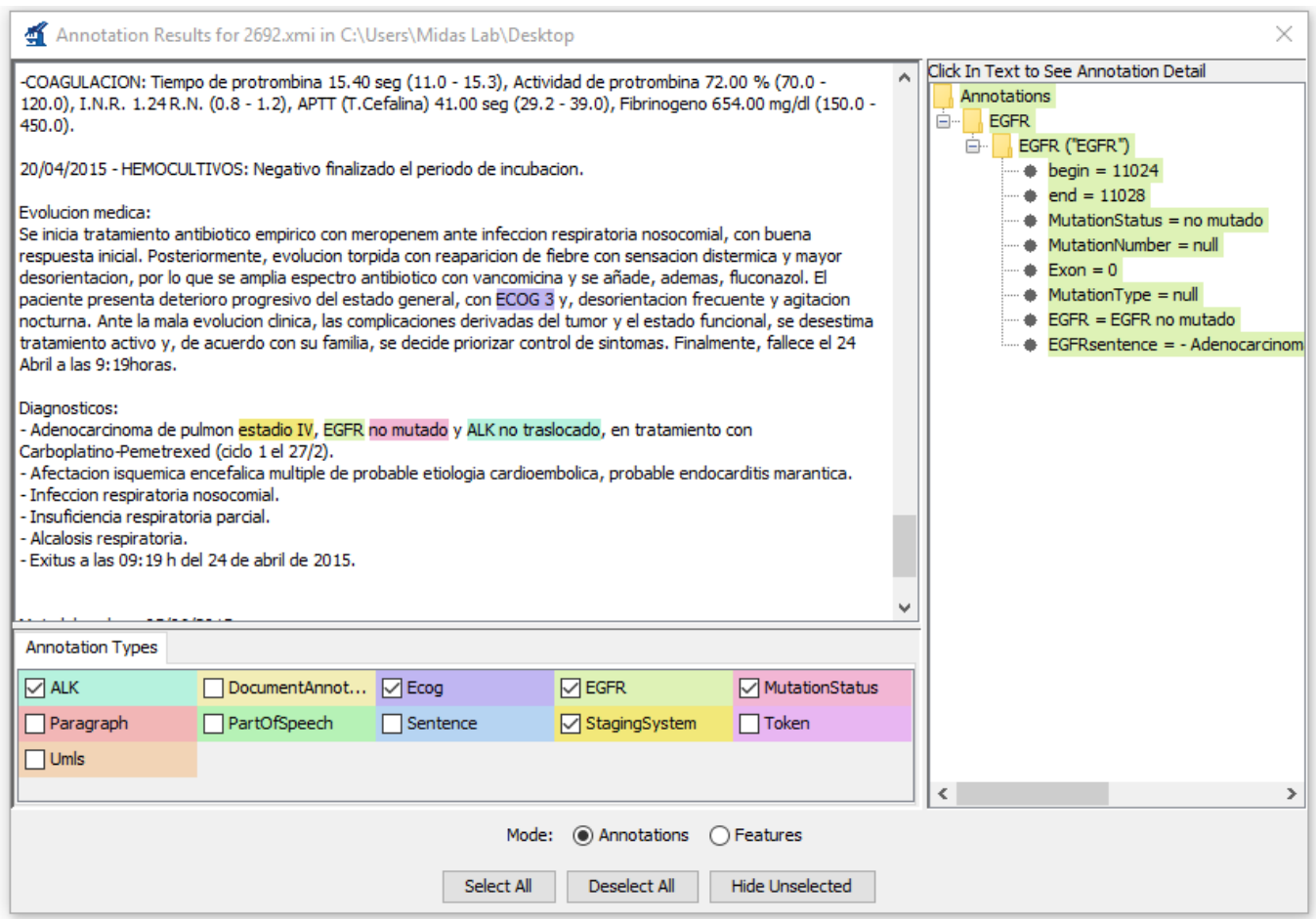

Figure 2.11: XMI annotation results on UIMA annotation viewer 
Chapter 2. Lung Cancer Concept Annotation from Spanish Clinical Narratives 2.5. Validation

\subsection{Validation}

To evaluate the performance of our developed annotators, we designed seven use cases for validating the output of:

1. EGFR Annotator

2. ALK Annotator

3. ROS1 Annotator

4. Stage Annotator

5. TNM Annotator

6. ECOG Annotator

7. Karnofsky Annotator

The following Section 2.5.1 discusses the details of the dataset used in these studies. Afterwards, Section 2.5.2 explains the details of the conducted experiments and Section 2.5.3 presents their results.

\subsubsection{Dataset}

In here, our objective is to describe the details of the dataset, the process of samples selection and the statistical tests performed to assess if the selected samples are representativeness of the entire population from the original dataset.

\section{Dataset Description}

The dataset used in our experiments includes information for 989 patients suffering from lung cancer. This dataset contains 296,003 EHRs, which are written in Spanish and are provided by the HUPHM of Madrid. The average number of EHRs per patient was 300. These EHRs contained the document creation date in structured format and were divided into two main 
Chapter 2. Lung Cancer Concept Annotation from Spanish Clinical Narratives 2.5. Validation

sources of data, clinical notes (corresponding to 281,308 EHRs) and clinical reports (14,695 EHRs). For the sake of clarity, throughout this Thesis, we collectively call these two sources EHRs, while other non-textual elements usually therein included (e.g., echography results) are disregarded for being outside the scope of this study. The average lengths of clinical notes and reports were approximately 550 and 2300 words, respectively. Figure 2.12 shows an overall view of the process of clinical notes and reports generation for a patient in HUPHM.

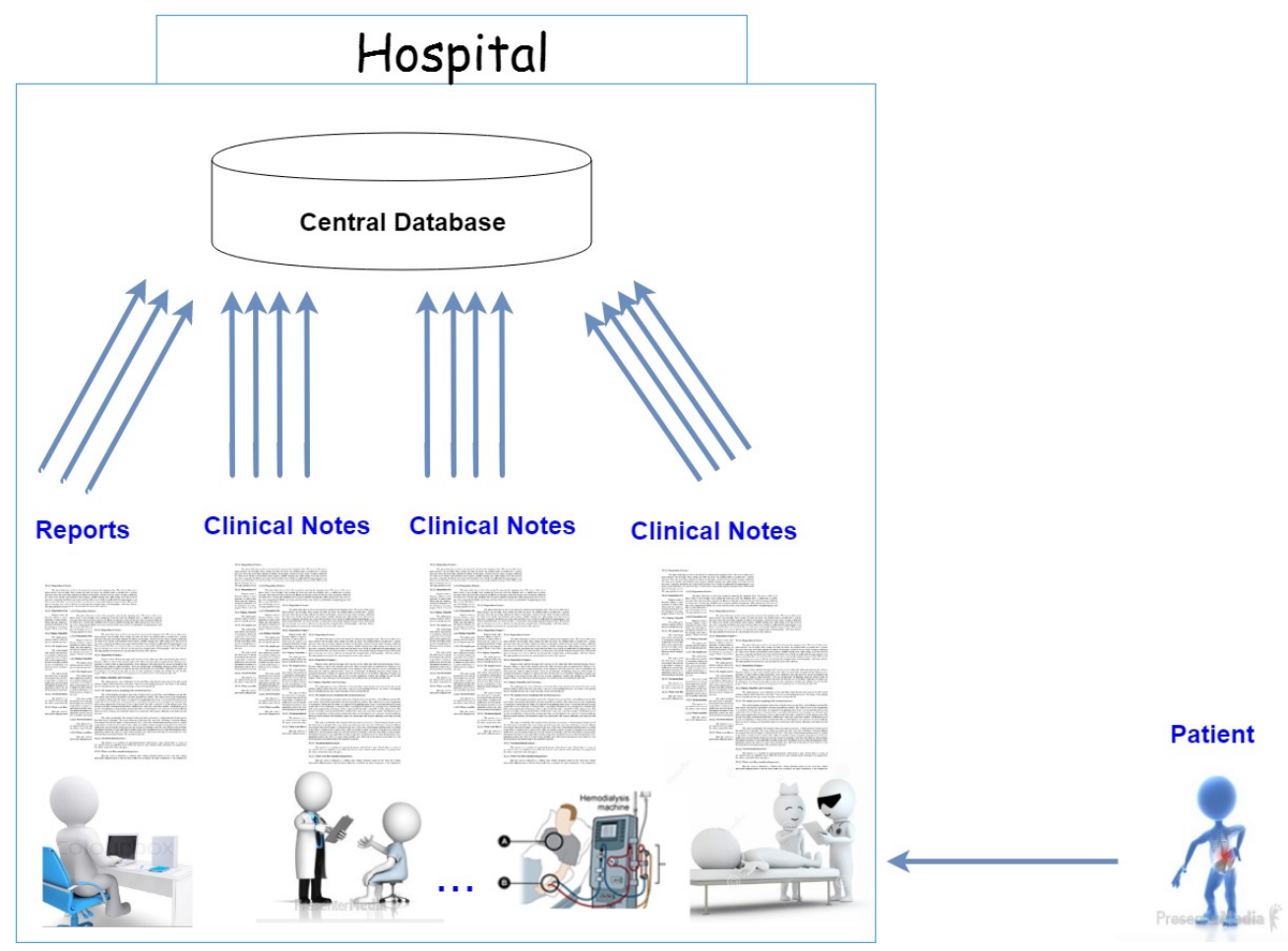

Figure 2.12: Process of clinical notes and reports generation for a patient in HUPHM

Clinical notes can be generated for a patient by different services and personnel in the hospital during each patient's visit at hospital. They are always written by a professional like physician, nurse, social service people, etc. They contain highly detailed information about the patient's personal and clinical status, processes followed and their results. 
Chapter 2. Lung Cancer Concept Annotation from Spanish Clinical Narratives 2.5. Validation

Figure 2.13 presents a sample of a clinical note. This clinical note begins with a semi-structured portion, which includes information about patient's ID (ID), date of birth (Fecha de nacimiento), gender (Género), date of starting the process (Fecha inicio proceso), state of the patient in hospital (Estado) and date of clinical note creation (Fecha). This is followed by an unstructured portion that captures the patient's clinical condition such as diagnosis of lung cancer (Adenocarcinoma de pulmón), tumor stage (estadío IIIA), TNM (T4N2M0), EGFR (EGFR negativo) and the health care provided to the patient (e.g., Dosis RT 26 Gy).

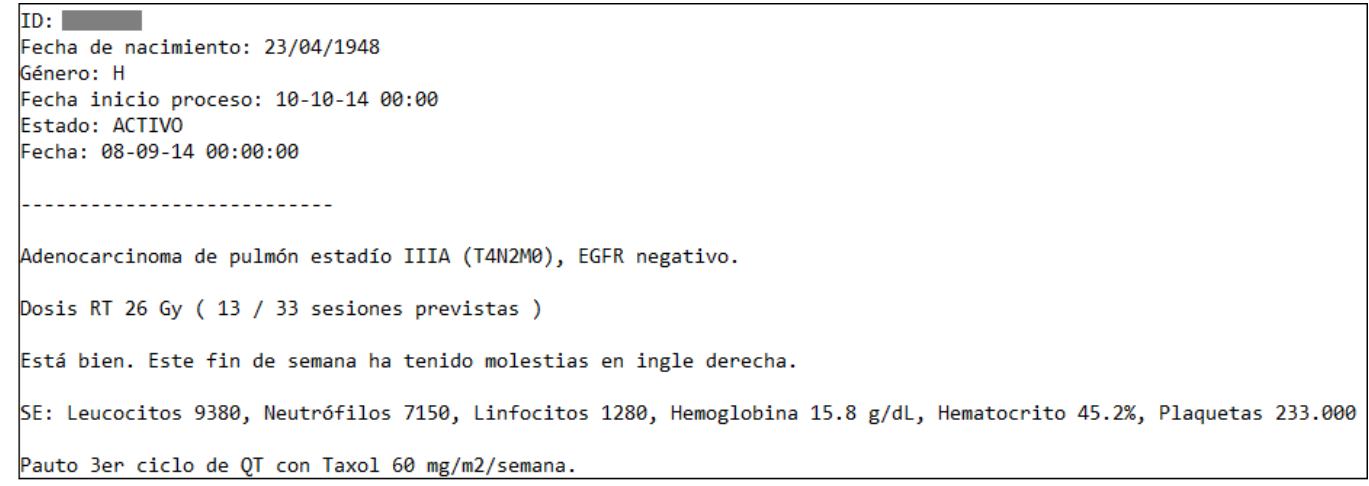

Figure 2.13: Example of a clinical note structure

On the other hand, clinical reports are generated when a medical process is completed, and they provide a summary of the corresponding clinical notes. Compared to the latter ones, they have a more structured format as they contain different sections (e.g., Personal History, Family Oncological History, Diagnosis, Treatment, etc.) under which the relevant information is provided.

Figure 2.14 presents a sample of a clinical report. Clinical reports starts with a semi-structured portion like the clinical note and then include different sections that capture patient's personal and clinical information. Such sections contain information about the service generated the clinical report (Servicio), the date of report creation (FECHA CREACION INFORME), the physician responsible for the clinical report (MEDICO RESPONSABLE INFORME), the patient's antecedents (Antecedentes personales), the oncological history (HISTORIA ONCOLÓGICA), the chest scan (TACDE 
Chapter 2. Lung Cancer Concept Annotation from Spanish Clinical Narratives 2.5. Validation

TÓRAX), the brainscopy (BRONCOSCOPIA), the principal diagnosis (Diagnóstico principal), the treatments (Tratamiento) and the recommendations (Recomendaciones).



Figure 2.14: Example of a clinical report structure 
Chapter 2. Lung Cancer Concept Annotation from Spanish Clinical Narratives 2.5. Validation

\section{Sample Dataset Selection}

Due to the huge amounts of EHRs provided by HUPHM, it was not feasible to perform a manual validation on the entire dataset. Therefore, to conduct our experiments, we have decided to perform individual processes of random sample selection of EHRs from the original dataset for each individual use case. The random sampling practice gave each element an equal chance of being selected.

For each use case, after conducting a study, we realized that their specific concepts have appeared in only less than $10 \%$ of EHRs - see Table 2.1. Due to low number of EHRs containing these concepts, the size of the random samples of EHRs which would yield statistically significant result was too large to be practical. We have therefore decided to randomly select individual samples of 550 EHRs from the original dataset for use case 1, 2, 4, 5 and 6 , such that 50 of them (including 25 clinical notes and 25 clinical reports) contain annotations extracted by the specific annotator, and 500 (including 250 clinical notes and 250 clinical reports) of them in which the annotator claimed there is no annotation in their clinical texts.

Table 2.1: Details of the selected samples for conducting experiments

\begin{tabular}{|c|c|c|c|c|c|c|}
\hline Use case & Annotator & $\begin{array}{c}\text { \% of } \\
\text { concept } \\
\text { appearance } \\
\text { in all the } \\
\text { EHRs }\end{array}$ & $\begin{array}{c}\text { No. of } \\
\text { randomly } \\
\text { selected } \\
\text { EHRs }\end{array}$ & $\begin{array}{c}\text { No. of } \\
\text { randomly } \\
\text { selected } \\
\text { clinical } \\
\text { notes }\end{array}$ & $\begin{array}{c}\text { No. of } \\
\text { randomly } \\
\text { selected } \\
\text { clinical } \\
\text { reports }\end{array}$ & $\begin{array}{c}\text { Selected } \\
\text { dataset } \\
\text { name }\end{array}$ \\
\hline 1 & EGFR & $4 \%$ & 550 & 275 & 275 & D1 \\
\hline 2 & ALK & $2 \%$ & 550 & 275 & 275 & D2 \\
\hline 3 & ROS1 & $0.64 \%$ & 1,100 & 550 & 550 & D3 \\
\hline 4 & Stage & $8 \%$ & 550 & 275 & 275 & D4 \\
\hline 5 & TNM & $9 \%$ & 550 & 275 & 275 & D5 \\
\hline 6 & ECOG & $1 \%$ & 550 & 275 & 275 & D6 \\
\hline 7 & Karnofsky & $0.01 \%$ & 1,100 & 550 & 550 & D7 \\
\hline
\end{tabular}

However, in case of use case 3 and 7 , since their specific concepts were mentioned in less than $1 \%$ of EHRs, we decided to increase the size of random sample. Hence, we have randomly selected individual samples of 1,100 EHRs from the original dataset, such that 100 of them (including 50 clinical notes and 50 clinical reports) contain annotations extracted by the specific annotator, and 1,000 (including 500 clinical notes and 500 clinical 
Chapter 2. Lung Cancer Concept Annotation from Spanish Clinical Narratives 2.5. Validation

reports) of them in which the annotator claimed there is no annotation in their clinical texts.

Note that, supposing independence in the errors incurred by the annotator, around 50 of these 500 negative results or 100 of 1000 negative results could be false negative, thus ensuring that both false positives and false negatives are tested with similar precision.

\section{Assessment of the Selected Sample Datasets}

A set of chi-squared statistical tests was performed on the selected samples to assess their representativeness of the entire population in the original dataset. These tests were performed on four significant variables of:

1. Patient's sex

2. Patient's age (categorical variable: <35, 35-45, 45-55, 55-65, 65-75, $75-80,80>)$

3. Local progression of the tumor

4. Systemic progression of the tumor

For all the samples and in all the use cases, the selected sample dataset was representative of the entire population ( $p$-value $<0.01$ ).

\subsubsection{Experiments}

Two computer scientists served as the evaluation domain experts in all the experiments under the supervision of clinicians from HUPHM. They were native Spanish speakers and participated neither in the design nor in the development of the annotators.

The evaluation of the annotators was done by manually analyzing the outputs generated by them. To validate each annotator, for each specific concept mentioned in the free texts of EHRs, a comparison was done between the list of the concepts automatically provided by the annotators and the list of expressions manually extracted by the evaluation domain experts. 
Chapter 2. Lung Cancer Concept Annotation from Spanish Clinical Narratives 2.5. Validation

Once the comparison is completed for each specific concept, the evaluation domain experts rated:

1. True Positive (TP): if the concept was correctly classified by the annotator.

2. False Positive $(\overline{\mathbf{F P}})$ : if the concept was incorrectly classified by the annotator.

3. Fasle Negative $(\overline{\mathbf{F N}})$ : if the annotator did not classify the concept when it should have.

Given TP, FP and FN, the standard precision, recall and F1 score were measured using the following equations:

$$
\begin{gathered}
\text { Precision }=\frac{T P}{T P+F P} \\
\text { Recall }=\frac{T P}{T P+F N} \\
F 1=\frac{2 \times P \times R}{P+R}
\end{gathered}
$$

In the case of precision and recall, confidence intervals were calculated by considering a binomial distribution with confidence level of $95 \%$.

\subsubsection{Results}

The summary of results from conducted experiments are shown in Table 2.2. Only Karnofsky Annotator obtained F1 score of 1. Other annotators have faced some errors in their annotation process. To identify the reasons of these errors, we have analyzed the output of the annotators extensively. The discussion of error analysis is provided in the following Sections. 
Chapter 2. Lung Cancer Concept Annotation from Spanish Clinical Narratives 2.5. Validation

Table 2.2: Validation results

\begin{tabular}{|c|c|c|c|c|c|c|} 
Use case & Annotator & Precision & $\begin{array}{c}\text { Confidence } \\
\text { interval for } \\
\text { precision }\end{array}$ & Recall & $\begin{array}{c}\text { Confidence } \\
\text { interval for } \\
\text { recall }\end{array}$ & F1 score \\
\hline 1 & EGFR & 1 & \pm 0.051 & 0.933 & \pm 0.082 & 0.965 \\
\hline 2 & ALK & 0.929 & \pm 0.076 & 0.929 & \pm 0.076 & 0.929 \\
\hline 3 & ROS1 & 0.97 & \pm 0.045 & 1 & \pm 0.028 & 0.985 \\
\hline 4 & Stage & 1 & \pm 0.048 & 0.872 & \pm 0.089 & 0.932 \\
\hline 5 & TNM & 0.961 & \pm 0.071 & 0.881 & \pm 0.089 & 0.919 \\
\hline 6 & ECOG & 0.903 & \pm 0.114 & 0.979 & \pm 0.09 & 0.94 \\
\hline 7 & Karnofsky & 1 & \pm 0.154 & 1 & \pm 0.154 & 1 \\
\hline
\end{tabular}

\section{Error Analysis for Use Case 1 - EGFR Annotator}

When we examined FNs, we realized that the main reason for occurrence of these errors are due to the ambiguous ways of writing the tumor mutation status in clinical texts. For example, the EGFR Annotator failed to annotate the EGFR mutation status is negative from "Ha negativizado el mutación".

\section{Error Analysis for Use Case 2 - ALK Annotator}

We have analyzed FPs and have seen that the main source for such errors are due to the mention of the mutation status for the ALK metric with other mutation status metrics (e.g., $A L K+R O S 1$ : no). However, FNs are the consequence of writing the value of mutation status without mentioning that it is referring to the ALK system (e.g., No se detecta ninguna mutación).

\section{Error Analysis for Use Case 3 - ROS1 Annotator}

By going through FPs generated by ROS1 Annotator, we have identified that the major reason for such errors is due to the failure of the annotator to recognize some of the negation cases (e.g., No se detecta ninguna mutación en $A L K$ y ROS1).

\section{Error Analysis for Use Case 4 - Stage Annotator}

By examining FNs, we realized that there are two main reasons for such errors. Firstly, this was due to ambiguous ways of writing tumor stage codes, i.e., writing the value of the tumor stage without mentioning that this value is referring to the stage of tumor. For example, the annotator failed to annotate " $I V$ " because there was no context word around it mentioning that this value 
Chapter 2. Lung Cancer Concept Annotation from Spanish Clinical Narratives 2.6. Discussion

is referring to tumor stage. Secondly, it also happened that the standard system for writing tumor stage codes was not used in clinical texts. For example, instead of "estádio IIIA", the clinicians wrote "estádio 3A".

\section{Error Analysis for Use Case 5 - TNM Annotator}

By analyzing FPs, we realized that main reason for such errors are because of using measures, which are not standard for mentioning TNM values in the text (e.g., cT4N1-2). Likewise, the analysis of FNs revealed that these errors occurred due to writing of TNM codes in combination with some explanations about the tumor stage given by the clinicians (e.g., $p$ T2a (pleura) pN1 (ganglio hiliar fragmentado; márgenes probablemente +) M0").

\section{Error Analysis for Use Case 6 - ECOG Annotator}

We have checked FPs and identified that while standard ECOG values should be a single number from 0 to 5 , it has been mentioned as a range value (e.g., ECOG 1-2). This caused the ECOG Annotator to extract incorrect values from clinical texts. In addition, only one FN error occurred due to usage of a non-standard system for writing ECOG metric. For example instead of the value zero for ECOG, the alphabet "O" was used.

\subsection{Discussion}

The vast amount of clinical data generated and the adaption of IT in the health care industry, have motivated the development of NLP systems in medical domain. For an NLP system to achieve a broad use, it must be capable of covering comprehensive clinical information and demonstrating effectiveness for a practical application.

Thus, in this Chapter, we have described the development of rule-based specific case annotators for lung cancer domain using UMIA framework. These annotators can detect information about tumor mutation status, stage of cancer and PS from clinical texts. The previous results obtained from executing these annotators support the idea that rule-based methods have 
Chapter 2. Lung Cancer Concept Annotation from Spanish Clinical Narratives 2.6. Discussion

shown an adequate level of performance in the NER process with F1 score above 0.90. Although some of these annotators have been developed general enough so that they can be used in other oncological domains but for them to be usable in other languages, the translation of the annotator's pattern to the designated language is required. 


\section{CHAPTER}

Recognition of Time Expressions in Spanish Electronic Health Records

The widespread adoption of EHRs is generating an ever-increasing amount of unstructured clinical texts. Processing time expressions from these domainspecific-texts is crucial for the discovery of patterns that can help in the detection of medical events and building the patient's natural history. In medical domain, the recognition of time information from texts is challenging due to their lack of structure; usage of various formats, styles and abbreviations; their domain specific nature; writing quality; and the presence of ambiguous expressions. Furthermore, despite of Spanish occupying the second position in the world ranking of number of native speakers, to the best of our knowledge, no NLP tools have been introduced for the recognition of time expressions from clinical texts, written in this particular language. Therefore, in this Chapter, we propose a Temporal Tagger for identifying and normalizing time expressions appeared in Spanish clinical texts. We further compare our Temporal Tagger with the Spanish versions of SUTime and HeidelTime. By using a large dataset comprising EHRs of people suffering from lung cancer, we show that our developed Temporal Tagger with an F1 score of 0.93 outperforms SUTime and HeidelTime with an F1 score of 0.797 and 0.85 , respectively. 
Chapter 3. Recognition of Time Expressions in Spanish Electronic Health Records 3.1. Introduction

\subsection{Introduction}

Time, the concept that mankind associates to the changes in the world, is crucial in biomedical informatics and EHRs 143 . Clinicians chronologically record the progress of a disease or a hospital course in clinical texts and store this information with time points in EHRs. To identify medical events and build the natural history of the patient, the retrieval of time expressions from clinical texts is thus essential.

In recent years, the annotation of time information has received a great attention by researchers due to the richness of time expressions, their importance in medical care, and the great availability of EHRs [33]. However, this information remains hidden within unstructured text of EHRs and requires the development of specific NLP techniques in order to be accessed.

Although a considerable amount of research has been performed on processing time expressions in clinical texts, most of the existing systems focus on English texts and perform their annotation with the help of annotated corpora. The creation of such corpora is firstly costly and time consuming, and secondly, their completeness directly affects the processing quality. In addition, while Spanish has acquired the second position in the world ranking of number of native speakers (with more than 572 million people) [20], to the best of our knowledge, no NLP system has been presented for processing time expressions in Spanish clinical texts.

Processing time information requires the ability to recognize its expressions and convert them from text to a normalized form, to simplify subsequent processing. Therefore, the main objective of this Chapter is to introduce a rule-based Temporal Tagger, capable of extracting and normalizing time expressions written in Spanish clinical texts. When compared with a standard alternative tools, i.e., the Spanish versions of SUTime [25,26] and HeidelTime [27,28], our solution presents a significantly higher F1 score, as we demonstrate by using a large collection of real EHRs. At the same time, it is both conceptually and technically simpler than machine learning solutions, as it does not require any annotated corpora. 
Chapter 3. Recognition of Time Expressions in Spanish Electronic Health Records 3.2. Problem Statement

The remainder of this Chapter is organized as follows. Firstly, the challenges associated with time expression extraction from clinical texts is discussed in Section 3.2. Then, the main related works are explained in Section 3.3 with focus on SUTime and HeidelTime, the main systems we used for benchmarking. Afterwards, the details of our developed Temporal Tagger for annotation of time expressions in Spanish clinical texts are described in Section 3.4, and its validation using a large collection of EHRs is presented in Section 3.5. Finally, Section 3.6 discusses the main results here presented and draws some conclusions.

\subsection{Problem Statement}

Within clinical context, time annotation presents four major challenges. First of all, the specific time notations can fall within three categories, i.e., natural (e.g. 5 days ago, representing $43 \%$ of time expressions in our dataset), conventional (e.g. 2011-09-16, 48\%) and professional time (e.g., 24hr, 9\%), each one presenting their own idiosyncrasies.

Secondly, physicians usually have limited time to write the details of patient-clinician encounters, and therefore have to resort to domain specific and non-standard expressions and abbreviations. This makes clinical texts hard to understand outside the medical community, let alone by automated systems. Furthermore, the presence of ambiguous expressions, having more than one semantical meaning in this domain-specific texts, adds an additional layer of complexity.

Thirdly, the interpretation of relative time expressions can be uncertain, being difficult to automatically identify which time point a relative time refers to. Note that, as opposed to standard texts, relative time expressions are here more prevalent compared with absolute ones.

Finally, granularity is an integral part of time information, both in absolute and relative expressions; it is thus not always clear what measurement unit 
Chapter 3. Recognition of Time Expressions in Spanish Electronic Health Records 3.3. State of the Art

should be adopted to represent different granularities. To illustrate, expressions like "5 days ago" or "September" are very common, and yet are difficult to decode in an exact way.

\subsection{State of the Art}

The objectives of this Section is to: (1) review the NLP systems developed for annotation of time expressions from free texts in Section 3.3.1 (2) provide information about those NLP systems introduced for annotation of time expressions from clinical texts in Section 3.3.2 and (3) describe the preliminaries required for extraction and normalization of time expressions with focus on SUTime and HeidelTime in Section 3.3.3.

\subsubsection{NLP Systems for Annotation of Time Expression from Free Texts}

The task of recognizing time expressions or simply TIMEX from free texts was first introduced in the Message Understanding Conference (MUC) in 1995 [144. Time expressions were tested as part of NER task, in which capitalized tokens were defined with one of the predefined semantic types such as Person, Location, Organization, Percentage, Money, Date and Time. The aim of this Section is to review the most important systems introduced for time expression annotation from English and Spanish free texts from the year 2000 onwards.

The Automated Content Extraction (ACE) 145, 146] started a series of evaluation campaigns with a major focus on Time Expression Recognition and Normalization (TERN) from English free texts by providing the International Organization for Standardization (ISO)-based date-time value. In TERN 2004, the Chronos system [147] obtained the F1 score of 0.872 for annotation of time expressions using rule-based methods.

The Detection And Normalization of Temporal Expressions (DANTE) system 148 was also developed to annotate time expressions from free texts and was evaluated on the ACE 2005 and ACE 2007 datasets. DANTE achieved the F1 score of $98.9 \%$ by implementing rule-based techniques. 
Chapter 3. Recognition of Time Expressions in Spanish Electronic Health Records 3.3. State of the Art

Time expression recognition and normalization from free-form texts have seen a great deal of interest with the development of the TimeML annotation schema [149] and release of TimeBank [150] newswire corpus. The TimeBank corpus was used in three temporal analysis evaluation tasks in the SemEval competitions, TempEval-1 [151], TempEval-2 [152], and TempEval-3 [153].

In Temp-Eval-2 for Spanish language, the Temporal Information Processing based on Semantic information (TIPSem) [31] used CRF models for extracting time expressions, and applied CRF and rule-based methods for normalizing time information. While TIPSem achieved the best F1 score of 0.91, TIPSem$\mathrm{B}$ and UC3M 154 obtained the second-best F1 score of 0.88. UC3M applied a rule-based approach for its implementations.

For English language in the context of TempEval-2, HeidelTime [155] gained the best $\mathrm{F} 1$ score of 0.86 by introducing a rule based system, which used regular expressions for extracting time expression and knowledge resources as well as linguistic clues for their normalization. TIPSem, TRIPS and TRIOS [156] were the second-best systems with F1 scores of 0.85 . TRIPS and TRIOS used a combination of deep semantic parsing, Markov Logic Networks, CRF classifiers and a set of rules for recognizing and normalizing time expressions.

A Perl temporal tagger was also developed as part of the TARSQI toolkit [157], named GUTime [158]. GUTime was built for processing time expressions appearing in English texts only. It was an extension of TempEx tagger [159], which handled both absolute and relative times and has been applied to different corpora, including broadcast news, print news, and meeting scheduling dialogs.

In addition, the Stanford university also built a rule-based temporal tagger, named SUTime [25, 26]. SUTime was developed upon regular expressions for recognizing and normalizing time expressions written in both English and Spanish texts. Both SUTime and GUTime were evaluated on English dataset of TempEval-2, achieving F1 scores of 0.92 and 0.84, respectively. Thus, SUTime outperformed the tools presented in TempEval-2 for annotation of time expressions. 
Chapter 3. Recognition of Time Expressions in Spanish Electronic Health Records 3.3. State of the Art

In TempEval-3, HeidelTime [27,28 was presented as a multi-lingual temporal tagger for extraction and normalization of time expressions mentioned in English and Spanish texts. For both languages, it achieved the highest F1 scores with values of 77.61 in English texts using HeidelTime-t and 90.1 in Spanish texts. In addition, the Spanish version of TIPSem, named as TIPSemB-Freeling (TIPSemB-F) was ranked as the second-best performing system with an F1 score of 87.4 in time expression tasks.

A time expression extraction system, known as ManTIME [160] carried the identification of English time expressions phase using machine learning techniques on a extensive set of features and a posteriori label adjustment pipeline to further improve the performance. It also followed the normalization phase using a set of rules. ManTIME was validated on the TempEval-3 dataset and achieved the F1 score of 1 in identification phase and accuracy of 0.77 for value attribute in the normalization phase.

The CogCompTime [161] was introduced as an NLP system to annotate time expressions from free texts, written in English. This system adapted a machine learning approach for extraction of time variables and implemented a rule parsing method for normalization of these variables. The TempEval-3 dataset was used for the evaluation of CogCompTime and the F1 scores of 84.9 and 84.7 was gained for the recognition and normalization of time expressions, respectively.

The Syntactic Time (SynTime [162] was also developed as an English rule-based tagger, which defined three main syntactic token types for grouping time-related regular expressions, namely time token, modifier and numeral. Time tokens were defined as words, which explicitly expressed time information (e.g., Year). In addition, modifiers were considered as tokens, which appeared before or after time tokens (e.g., Ago, Several) and modify them. Further, numerals were determined as ordinals or numbers. SynTime was evaluated on TimeBank corpus and gained F1 scores of 92.47 and 94.62 for strict and exact match tasks, respectively.

In general, numerous systems have been proposed for processing time expressions in both English and Spanish texts using rule-based and machine learning approaches. However, these systems are not generally flexible, i.e., 
Chapter 3. Recognition of Time Expressions in Spanish Electronic Health Records 3.3. State of the Art

they are not designed to work with the various styles and formats a date can be written in.

Furthermore, the systems developed for processing time information from free texts in the general domain may not be efficient enough to be applied to the medical one. As discussed in [143], this statement is theoretically supported by the sub-language theory, which shows that a restricted domain is more well-defined than a general one, and can more accurately be characterized by specific vocabularies, semantic relations and, in some cases, syntax.

\subsubsection{NLP Systems for Annotation of Time Expression from Clinical Texts}

In this Section, we review the most significant systems presented for identification of time expressions from English clinical texts from the year 2000 onwards. In medical domain, the extraction and normalization of time expressions have been the topic of many shared tasks over the past few years. Among them, it is worth highlighting the Integrating Biology and the Bedside (i2b2) NLP Challenge 163], clinical Temp-Eval 2015 [164], clinical Temp-Eval 2016 [165] and clinical Temp-Eval 2017 [166.

The i2b2 NLP Challenge provided the researchers with an English corpus of discharge summaries, which was annotated with temporal information in the year 2012 [167]. Using ISO-TimeML annotation guidelines and by implementing the regular expressions and machine learning methods, researchers were able to perform extraction and normalization of time information from clinical texts. Among all participants, the Mayo clinic system achieved the highest accuracy of 0.73 by using regular expressions for performing the time expression tasks.

In 2014, an extension of ISO-TimeML guidelines was developed to annotate an English corpus of clinical notes, provided by the Mayo clinic, known as Temporal Histories for Your Medical Events (THYME) corpus [168]. This dataset has hitherto been used in several competitions. In clinical Temp-Eval 2015, BlueLab [169 used SVM classifiers with features generated by the Apache cTAKES [100, 101]. It obtained the highest F1 score of 0.709 for 
Chapter 3. Recognition of Time Expressions in Spanish Electronic Health Records 3.3. State of the Art

identifying the span and class (DATE, TIME, DURATION, QUANTIFIER, PREPOSTEXP or SET) of time expressions.

In addition, in clinical Temp-Eval 2016, UTHealth [170 implemented linear and structural (HMM) SVMs using lexical, morphological, syntactic, discourse, and word representation features. Its run 1 gained the best F1 score of 0.772 for span and class of time information.

In the context of clinical Temp-Eval 2017, KULeuven-LIIR [171] used linear SVM classifiers with features including words and part-of-speech to find time expressions mentioned in clinical texts. KULeuven-LIIR achieved the highest F1 score of 0.53 for time span and class information in Unsupervised domain adaptation. Nevertheless, within the specific task of supervised domain adaptation, GUIR [172] achieved the best F1 score of 0.56 for time span and class information. GUIR used supervised learning algorithms with lexical, syntactic, semantic, distributional, and rule-based features.

The NLP system of cTAKES was also extended with a temporal module, employing forward and backward search algorithms and multiple learning methods, like SVM and CRF, for annotation of time expressions from clinical narratives written in English [173, 174.

In addition, the Temporal Expression ExtractoR (TEER) 175 was introduced as a method for extracting and normalizing English and Chinese time expressions from different types of clinical texts. TEER integrated the heuristics rule generation and the automatic pattern learning for its implementation. It achieved F1 score of 0.911 for annotation of time expressions from English clinical texts.

For medical domain, most of the state-of-the-art systems were developed using annotated corpora, provided in English by the shared tasks. Yet, the limited size of such corpora can significantly affect the quality of the processing. In addition, to the best of our knowledge, no system has been proposed for Spanish. 
Chapter 3. Recognition of Time Expressions in Spanish Electronic Health Records 3.3. State of the Art

\subsubsection{Preliminaries}

As we here benchmark our proposed Temporal Tagger against the Spanish versions of SUTime and HeidelTime, for the sake of completeness, in this Section, we provide an overview of the structure and performance of the latters.

SUTime, a pattern-based extraction annotator, is basically developed for retrieval of time information from English free texts. It supports four basic types of temporal expressions, namely TIME, DURATION, INTERVAL and SET. This annotator is implemented under the Stanford CoreNLP pipeline [176], which supports tokenization of free texts, making it convenient for SUTime to specify regular expression over tokens.

Given a tokenized text, to extract time expressions SUTime follows a three-fold strategy:

1. Building patterns over individual words for recognizing numeric expressions.

2. Using patterns over words and numerical expressions to recognize simple time expressions.

3. Forming compound patterns on discovered temporal expressions.

Once time expressions are recognized, ambiguous expressions that are not likely to be time-related are removed from the list of candidates. Then, each of the expressions is associated to a temporal object. If there is a relative temporal object, it will be resolved based on document date. Also, if there is a confusion about the time point which the relative temporal object refers to (e.g., Monday), the verb tense of the clause is used to help in resolving the ambiguity. Finally, SUTime performs the internal time representation of all the temporal objects and produces a TIMEX3 [149] annotation for each temporal objects. At a later stage, SUTime was extended for annotation and normalization of TIME expressions written in Spanish. 
Chapter 3. Recognition of Time Expressions in Spanish Electronic Health Records 3.4. Solution

HeidelTime is also a rule-based system, which makes use of regular expressions for annotation of time expressions. It is developed as an UIMA component for extraction and normalization of TIME, DURATION, SET and DATE types of temporal expressions. For relative time expressions, it uses the date of previously mentioned time expression in the text, if any otherwise, it makes use of document creation date. At the end, it generates TIMEX3 annotations as output for each time expression mentioned in the texts.

Finally, both SUTime and HeidelTime annotators contain a limited set of rules for performing the NER process in Spanish, does not support the disambiguation feature for removal of expressions that are not likely to be related to time, and does not deal with ambiguities about the time to which a relative expression object may refer.

\subsection{Solution}

Every time expression $t$ can be viewed as a two-tuple:

$$
t=(t i, v i)
$$

where $t i$ is the time expression itself and $v i$ is the normalized value. Our goal is to extract every time expression $t i$ and to accurately assign the value attribute $v i$. For this purpose, we developed a rule-based NLP module using the Apache UIMA framework, named Temporal Tagger. This Tagger is built based on the DATE type of TIMEX3 tag in TimeML annotation guidelines and by manually studying EHRs.

Given a tokenized and PoS labeled text, the Temporal Tagger is capable

of processing time expressions, written in Spanish, within clinical texts. To provide the temporal tagger with a tokenized and PoS labeled text, we have used the NLP pipeline of C-liKES 22$]$.

The main steps of our Temporal Tagger are:

1. Extraction of various time expressions 
Chapter 3. Recognition of Time Expressions in Spanish Electronic Health Records 3.4. Solution

2. Filtration of time expressions

3. Resolution of time expressions with respect to a reference date

4. Normalization of time expressions to a standard date format

The detailed information about each one of these steps is provided in the following Sections 3.4.1, 3.4.2, 3.4.3 and 3.4.4.

\subsubsection{Extraction of Various Time Expressions}

Given a tokenized text, the Temporal Tagger identifies time expressions and outputs annotations for further manipulations and interpretations. Its output includes annotations in form of XMI files.

The Temporal Tagger is capable of structuring natural (e.g., 5 days ago and Today), conventional (e.g., September sixteen, 2011) and professional (e.g., 24hr) time expressions, the three common ways in which a date can be written in Spanish.

It also supports annotation of time expressions written in different formats and styles. For instance, some of them include DD-MM-YYYY, MM-DDYYYY, YYYY-MM-DD, YYYY-DD-MM, DD-MM-YY, MM-DD-YY, The day DD of the month MM of the year YYYY, etc. Since our Temporal Tagger is optimized for Spanish, in which the standard time expression is written as DD-MM-YYYY or YYYY-MM-DD, we assign priority to these two rules over the alternative MM-DD-YYYY and YYYY-DD-MM. In addition, by various styles we mean numerical, alphabetical, mixed (alphabetical and numerical) or even abbreviated time expressions.

For example, the date "16/09/2011" can be written in different formats and styles as "09/16/2011", "16-9-2011", "The day 16 of the month 9 of the year 2011", "September sixteen, 2011", "Sep 16, 2011" etc. Finally, the Temporal Tagger supports the extraction of time points, indicating a particular instance on a time line. 
Chapter 3. Recognition of Time Expressions in Spanish Electronic Health Records 3.4. Solution

\subsubsection{Filtration of Time Expressions}

Given a text labeled with PoS tags, the Temporal Tagger removes those time expressions from the list that are not likely to be such. For example, the word "Tarde" has two meanings in Spanish, "Afternoon" and "Late". In this specific case, we specified a rule according to which, if a candidate time expression is a single word "Tarde" and its PoS tag is not a noun, then it is not referring to a time point; hence, the Temporal Tagger ignores from annotating it as a time expression.

\subsubsection{Resolution of Time Expressions with Respect to a Reference Date}

Processing relative time expressions (e.g., "5 days ago") requires a reference date on which the statement was made. The Temporal Tagger uses the section date (if any) or the document date of the EHR as references. For example, for an EHR with the document date of "16/09/2011", the Temporal Tagger would resolve the date referred to by "5 days ago" into "11/09/2011".

However, there could be confusion about the time point which an expression refers to. For example, for the time expression "Tuesday" from a reference date like "16/09/2011", it is not clear whether it refers to "13/09/2011" (i.e., in the past) or "20/09/2011" (i.e., in the future). In this case, the verb tense of the sentence is used to resolve the ambiguity. However, since the clinical text is more compact and may not include a verb and the clinical narratives mostly provide information about past, then "Tuesday" refers to "2011-09-13" by default.

\subsubsection{Normalization of Time Expressions to a Stan- dard Date Format}

Once the time expressions have been identified and processed, the Temporal Tagger normalizes them into the standard date format of YYYY-MM-DD. For example, for an extracted time expression of "September sixteen, 2011", the Temporal Tagger converts the date to "2011-09-16".

However, if the month and/or the day of the time expression is not identified, the Temporal Tagger automatically assigns the value 00 to the corresponding 
Chapter 3. Recognition of Time Expressions in Spanish Electronic Health Records 3.5. Validation

MM or DD value. Note that this value is used to record the uncertainty about the exact date. For example, for the time expression "September, 2011", the Temporal Tagger would convert the date to "2011-09-00". In Table 3.1, we give examples of some expressions recognized and normalized by our Temporal Tagger.

Table 3.1: Example of time expressions annotated by our Temporal Tagger (with 2016-12-23 as reference date)

\begin{tabular}{|l|l|}
\hline \multicolumn{1}{|c|}{ Time expression } & $\begin{array}{c}\text { Normalized } \\
\text { value }\end{array}$ \\
\hline $16 / 09 / 2011$ & $2011-09-16$ \\
\hline September sixteen, 2011 & $2011-09-16$ \\
\hline 5 days ago & $2016-12-18$ \\
\hline Today & $2016-12-23$ \\
\hline The day after tomorrow & $2016-12-25$ \\
\hline Tuesday night & $2016-12-20$ \\
\hline Last night & $2016-12-22$ \\
\hline September & $2016-09-00$ \\
\hline 2011 & $2011-00-00$ \\
\hline 24 hour & $2016-12-22$ \\
\hline
\end{tabular}

\subsection{Validation}

The evaluation of our developed Temporal Tagger consists of two use cases:

1. Validation of the output of the Temporal Tagger

2. Comparison of the Temporal Tagger with the Spanish versions of SUTime and HeidelTime

Section 3.5.1 discusses the details of the dataset used in these studies, Section 3.5 .2 explains the conducted experiments and Section 3.5 .3 presents their results. 
Chapter 3. Recognition of Time Expressions in Spanish Electronic Health Records 3.5. Validation

\subsubsection{Dataset}

To conduct our experiments, we used the dataset given by HUPHM, which is explained in Section 2.5.1. The following Sections discuss the process followed for sample selection and explains the details of the statistical tests performed on the selected sample.

\section{Sample Dataset Selection}

Due to the huge amounts of EHRs provided by HUPHM, it was not feasible to perform a manual validation on the entire dataset. Therefore, to conduct our experiments, we have decided to perform a random selection of 100 EHRs from the original dataset, including 50 clinical notes and 50 clinical reports. The selection of equal number of notes and reports was aimed at keeping both types of clinical documents significantly present in the validation phase.

\section{Assessment of the Selected Sample Dataset}

Using chi-squared tests, we have performed a set of statistical tests on the selected sample to assess its representativeness of the entire population in the original dataset. These chi-squared tests were performed on:

1. Patient's sex

2. Patient's age (categorical variable: $<35,35-45,45-55,55-65,65-75$, $75-80,80>)$

3. Stage of tumor

4. Local progression of the tumor

5. Systemic progression of the tumor

In all cases, except for the systemic progression, the sample was representative of the entire population ( $\mathrm{p}$-value $<0.01$ ). However, a small amount of bias was observed in the systematic progression of tumor between the patients of the selected sample and the patients of dataset; note that this is not expected to affect the types of temporal expressions found. 
Chapter 3. Recognition of Time Expressions in Spanish Electronic Health Records 3.5. Validation

\subsubsection{Experiments}

Two computer scientists, who were native Spanish speakers, served as evaluation domain experts under the supervision of clinicians from the HUPHM and helped in conducting the experiments. None of them participated in the design or development of the Temporal Tagger.

\section{First Use Case - Validation of the Output of the Temporal Tagger}

In our first use case, the evaluation was done by manually analyzing the output provided by the Temporal Tagger. For each time expression written in the free text of EHRs, a comparison was done between the list of the expressions automatically provided and the list of expressions manually extracted. After the comparison was completed, confusion matrices were calculated for then obtaining the TP, FP and FN values, in order to determine precision, recall and F1 score values. To obtain TP, FP and FN, for each time expression appeared in the clinical texts, the evaluation domain experts rated:

1. TP: if the time expression was correctly classified and normalized by the Temporal Tagger.

2. FP: if the time expression was incorrectly classified or normalized by the Temporal Tagger.

3. FN: if the Temporal Tagger did not classify the time expression when it should have.

Given TP, FP and FN, we calculated precision, recall and F1 score. In case of precision and recall, confidence intervals were calculated by considering a binomial distribution with confidence level of $95 \%$.

\section{Second Use Case - Comparison of the Temporal Tagger with the Spanish Versions of SUTime and HeidelTime}

The idea of our second use case it to compare our solution with existing ones, specifically the Spanish versions of SUTime and HeidelTime. By implementing the first use case, the results of our Temporal Tagger were obtained. To measure the accuracy of SUTime and HeidelTime for retrieval of time variables, the first use case was also repeated by the evaluation domain experts on the 
Chapter 3. Recognition of Time Expressions in Spanish Electronic Health Records 3.5. Validation

same dataset of 100 EHRs. Finally, the results were manually analyzed, and a comparison was performed between our Temporal Tagger with SUTime and HeidelTime.

\subsubsection{Results}

The results of the first use case show that the Temporal Tagger achieves a precision of $0.927 \pm 0.021$, recall of $0.932 \pm 0.021$ and F1 score of 0.93 . To find the errors occurred in the annotation by our Temporal Tagger, we analyzed its output extensively.

By examining FPs, we realized that the majority of errors are caused by incorrect normalization of relative time expressions. The main reasons for such errors were:

1. Usage of compact clinical sentences without mention of verbs in them. As a result, the Temporal Tagger referred the relative time expressions to the past time while they were referring to the future time.

2. Existence of ambiguous time variables such as the the noun "Mañana", which has two meanings in Spanish, "Morning" and "Tomorrow". This leads to incorrect normalization of these time variables in some cases.

3. few errors occurred due to the limitation of the Temporal Tagger to cover time durations like "This week" or "This year".

The analysis of FNs revealed that most of the errors were the consequence of mentioning combined time expressions (e.g., 1, 4, 8 and 14-sep) and the usage of dots "." within time expressions (e.g., 17.1.14). As the C-liKES tokenizer uses dots as the indicators of end of a sentence, this precludes the Temporal Tagger to capture the complete pattern.

The results of our second use case are discussed here. As it can be seen in Figure 3.1, the experimental results show that our Temporal Tagger outperformed both SUTime and HeidelTime in terms of precision, recall and F1 score. While SUTime obtained the precision of $0.831 \pm 0.033$, recall of $0.766 \pm 0.036$ and $\mathrm{F} 1$ score of 0.797 , HeidelTime achieved the precision of 0.795 \pm 0.032 , recall of $0.915 \pm 0.025$ and F1 Score of 0.85 . The main differences were 
Chapter 3. Recognition of Time Expressions in Spanish Electronic Health Records 3.5. Validation

found in the recognition of various formats a time expression can be written as well as in annotation of natural and professional time expressions. Our Temporal Tagger had a better performance in processing such time variables compared to both SUTime and HeidelTime.

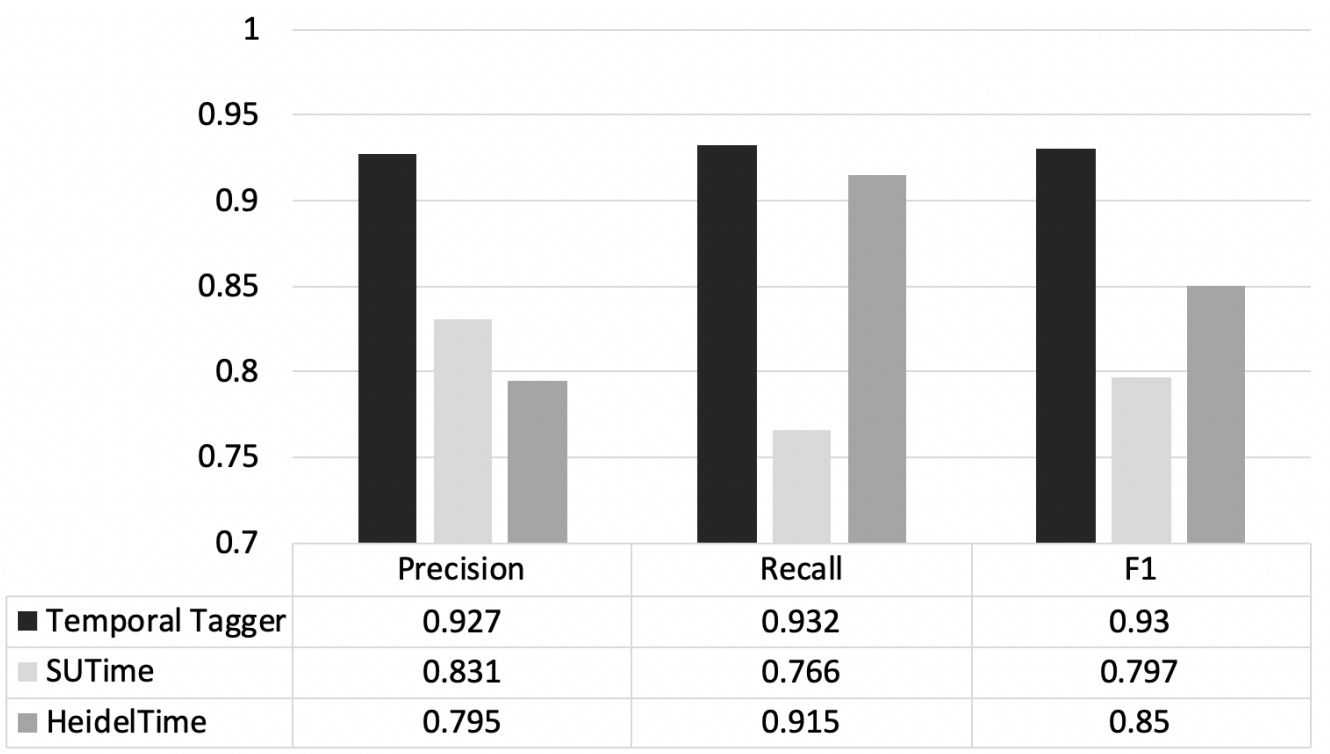

Figure 3.1: Comparison of the results of our Temporal Tagger with SUTime and HeidelTime

Regarding the detection of numerical conventional time expressions, all the taggers have shown closely equal performances but for the annotation of mixed and abbreviated conventional time variables, the Temporal Tagger showed a higher accuracy. In addition, by assigning priorities to the rules of DD-MM-YYYY and YYYY-MM-DD and by performing filtration, our Temporal Tagger obtained more precise normalization results compared to both SUTime and HeidelTime . 
Chapter 3. Recognition of Time Expressions in Spanish Electronic Health Records 3.6. Discussion

\subsection{Discussion}

Annotation of time expressions is essential to the task of investigating problems requiring temporal information, such as event extraction and temporal ordering of events. Before using time expressions in any other applications, the first step is to extract and normalize time variables appearing in free texts. Therefore, in this Chapter, we presented a Temporal Tagger, capable of processing time information appearing in Spanish clinical texts. As one of our primary objectives was to yield a generic Temporal Tagger that could be applied in other scenarios with minimal adaptions, it has been developed as an UIMA component.

In synthesis, all previous results support the idea that our Temporal Tagger had higher precision, recall and F1 score than the Spanish versions of SUTime and HeidelTime in retrieving time expressions from clinical texts. We applied rule-based approaches for extraction and normalization of time variables similar to latters.

We observed that by supporting regular expressions for annotation of various styles and formats a time variable can be written in, and by supporting natural, conventional and professional time expressions, our Temporal Tagger has obtained a high accuracy. In addition, the assignment of priorities to some rules for Spanish language and the processes of filtration and resolution with respect to a reference date played a great role in providing accurate normalized values.

However, we have also seen that the relative time expressions were the most difficult ones for our Temporal Tagger to normalize. Also, the tagger faced some errors due to its limitation to annotate the duration, the combined time expressions and those time variables that used dot in their format, like DD.MM.YYY.

Our aim was to benchmark the existing SUTime and HeidelTime tools, and the rule-based methods to annotate time expressions from clinical texts written in Spanish. Adaptions of machine learning based methods requires annotated corpora, whose building is both time-consuming and costly. In 
Chapter 3. Recognition of Time Expressions in Spanish Electronic Health Records 3.6. Discussion

addition, the small size of their annotated corpora could unavoidably affect the processing quality. 


\section{СHAPTER 4}

\section{Reconstructing the Patient's Natural History from Electronic Health Records}

The automatic extraction of a patient's natural history from EHRs is a critical step towards building intelligent systems that can reason about clinical variables and support decision making. Although EHRs contain a large amount of valuable information about the patient's medical care, this information can only be fully understood when analyzed in a temporal context. Any intelligent system should then be able to extract medical concepts, date expressions, temporal relations and the temporal ordering of medical events from the free texts of EHRs; yet, this task is hard to tackle, due to the domain specific nature of EHRs, writing quality and lack of structure of these texts, and more generally the presence of redundant information. In this Chapter, we introduce a new NLP framework, capable of extracting the aforementioned elements from EHRs written in Spanish using rule-based methods. We focus on building medical timelines, which include disease diagnosis and its progression over time. By using our large dataset of EHRs comprising information about patients suffering from lung cancer, we show that our framework has an adequate level of performance by correctly building the timeline for 843 patients from a pool of 989 patients, achieving a precision of 0.852 . 
Chapter 4. Reconstructing the Patient's Natural History from Electronic Health Records 4.1. Introduction

\subsection{Introduction}

The treatment of a disease does not only depend on the current condition of a patient, but also on his/her past medical history. This is why it is crucial for clinicians to have a complete and precise knowledge of the patient's natural history, which includes the disease, its progression over time, and any other significant fact in chronological order.

As largely recognized in literature and as mentioned in Chapter 1, retrieving the patient's natural history can help improving clinical document summarization, clinical trial recruitment, clinical decision making, and patient's survival time calculation. In addition, accessing this information allows clinicians to evaluate the quality of the provided healthcare, and to identify which of its steps require a special attention.

If such clinical information has traditionally been managed and accessed manually, the last decade has witnessed an increasing need for the digitization of clinical data. For this purpose, the information about the interactions between a patient and clinicians is frequently stored in computerized clinical records, which allow the reconstruction of the patient's natural history - see Figure 4.1 for a graphical representation.

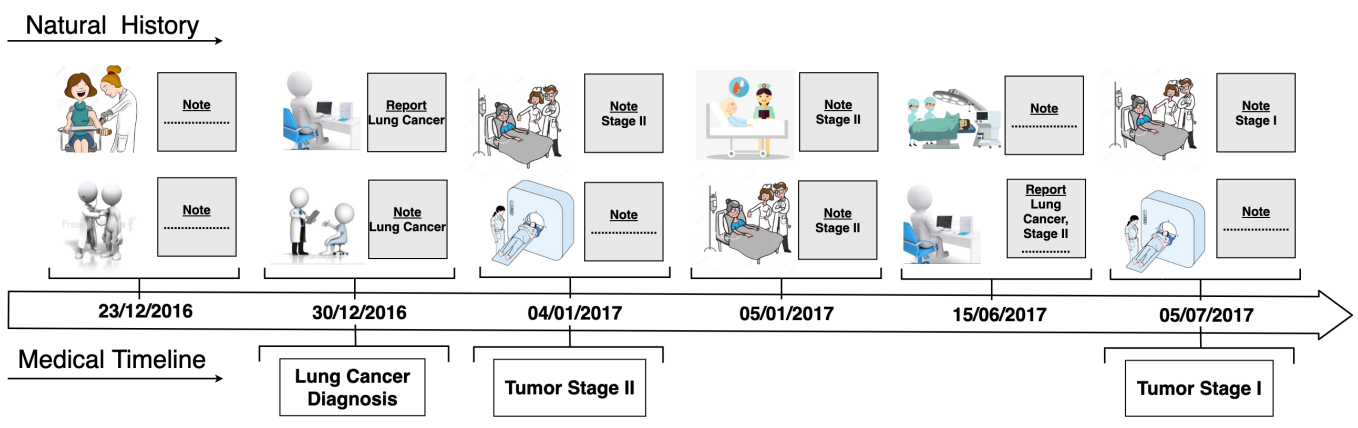

Figure 4.1: An example of a natural history of a patient suffering from lung cancer (top part), and the corresponding medical timeline for disease diagnosis and progression (bottom part)

Whenever a patient visits a hospital, one or more clinical notes can be digitally generated, describing highly detailed information about the patient's 
Chapter 4. Reconstructing the Patient's Natural History from Electronic Health Records 4.1. Introduction

past and present medical condition, diagnosis, disease progressions, treatments, lab test results, etc. Note that, while digital in nature, notes are mostly composed of free texts and are therefore unstructured. These clinical notes are always written by a professional (physician, nurse, etc.).

Complementary to notes, clinical reports are digitally generated once a medical process is completed, and they consolidate and synthesize the information contained in several clinical notes. They have a more structured format compared to the latter ones by containing different sections (e.g., Family Oncological History, Personal History, Diagnosis, Treatment, etc.) under which the relevant information is provided. EHRs are therefore unstructured clinical documents describing various medical events related to the patient's clinical condition and the corresponding chronological sequence.

Although EHRs contain all the information needed to reconstruct the patient's natural history, their manual analysis can be both costly and timeconsuming. Oncology provides an ideal case study to show the importance of automatic EHR processing. The different risk factors of cancer, the intratumor heterogeneity that implicates the patient's differences in relation to the same cancer type, and that the same patient presents differences between tumor sites, the inter-tumor heterogeneity, the differences in treatment response for the same cancer among patients, and the substantial difficulties in predicting tumor dynamics and the associated outcomes are some of the challenges that oncologists have to face in the daily clinical practice.

When the objective becomes the design of a personalized treatment, big data analytics becomes the instrument of choice to tackle this heterogeneity and variability; in turn, this requires the extraction and processing of information coming from EHRs.

One of the types of cancer with higher prevalence and higher mortality worldwide, which encounters all of these difficulties is lung cancer. This is mainly due to the fact that its diagnosis is made in most cases in advanced stages of the disease where surgery is not an option anymore and the tumor burden is high. 
Chapter 4. Reconstructing the Patient's Natural History from Electronic Health Records 4.1. Introduction

Diagnosis of lung cancer is usually accidental, during a visit to the emergency department when an image test is performed, and a lung nodule or mass is detected. From this moment, the patient should visit the medical oncology service, where the oncologist will confirm the diagnosis with additional tests if necessary, in order to subsequently receive the most suitable treatment available. Depending on the treatment and its frequency, the patient will visit accordingly both the consultation and the day care hospital where they receive treatments, along with frequent blood tests, imaging tests, and routine controls by the oncology nurses.

In terms of data generation, especially in lung cancer patients, the exploitation of this variety of data stored in EHRs from all lung cancer patients may lead to pattern extraction and better understanding of the disease evolution, toxicity rates and treatment response and outcomes. However, the process of reconstructing a patient's natural history from EHRs requires the extraction of several key elements, like medical concepts, date expressions, temporal relations and the order of medical events from free texts where medical events are medical concepts linked to their occurrence date expressions.

If EHRs contain all the information needed to reconstruct the patient's natural history, their manual analysis can be both costly and time-consuming. To illustrate, a typical lung cancer treatment can be composed of hundreds of notes and reports, summing up tens of thousands of words - see Section 2.5.1 for details on the real data set here considered.

It is therefore not a surprise that a great interest has emerged on the automatic identification of timelines of medical events starting from patient EHRs [177]. Such reconstruction process requires the extraction of several key elements, like medical concepts, time expressions, temporal relations and the order of medical events from free texts.

Although a great amount of research works has been done on identifying temporal relations from clinical texts, the performance of most proposed systems is far from being adequate for practical applications. Most of these systems perform temporal reasoning with the help of annotated corpora, which are time consuming and costly to build, and whose completeness affects the quality of the analysis. 
Chapter 4. Reconstructing the Patient's Natural History from Electronic Health Records 4.1. Introduction

In addition, despite the fact that Spanish is the second most popular language in the world with more than 572 million native speakers [20], little attention has been devoted to temporal relation discovery from Spanish free texts in the general domain. Finally, and to the best of our knowledge, no system has hitherto been proposed for the discovery of temporal relations from Spanish clinical texts.

The aim of this Chapter is to present a novel NLP framework, using rulebased techniques, that firstly, accepts Spanish EHRs annotated with medical concepts and date expressions as input, and then is able to relate these concepts together for reconstructing the patient's natural history. While, we aim to link medical concepts to the document creation dates, section dates and within-sentence dates in EHRs, identification of temporal relations between pairs of medical concepts are disregarded for being outside the scope of this study.

In addition, in this Chapter, we propose to go one step further by extracting the evolution of medical events from these clinical documents, in order to build the patient's medical timeline, which contain the diagnosis of the disease and its progression over time. In particularly, we have applied this framework to generate the medical timelines of disease diagnosis and tumor stage events for patients suffering from lung cancer. Our framework presents a remarkable performance, yielding a precision of 0.852 , as validated by using a large set of real EHRs.

Finally, in order to provide our NLP framework with the input of annotated lung cancer diagnosis concepts, tumor stage codes and date expressions, we have used a set of annotators, which are developed over UIMA framework. These annotators along with the temporal relation identification process is facilitated with the tokenizer and section recognizer of C-liKES [22].

The rest of Chapter is organized as follows. Firstly, Section 4.2 discusses the problem statement. Then, Section 4.3 explains the main related works on temporal relation discovery and patient's medical timeline reconstruction. Afterwards, Section 4.4 discusses the details of the proposed framework by providing solutions for identifying temporal relations and building the patient's medical timeline. Section 4.5 presents the validation of the framework by 
Chapter 4. Reconstructing the Patient's Natural History from Electronic Health Records 4.2. Problem Statement

using a real data set. Finally, Section 4.6 discusses the main advantages and limitations of the proposed framework.

\subsection{Problem Statement}

The process of reconstructing the patient's natural history from their EHRs, entails two main categories of challenges: (1) linkage of medical events to time expressions, discussed in Section 4.2.1 and (2) derivation of the order of medical events from the patient's EHRs, discussed in Section 4.2.2.

\subsubsection{Challenge 1 - Linkage of Medical Events to Time Expressions}

Languages like English and Spanish use the interplay of tense and aspect to encode temporal relations. However, the significance of these features may vary across domains and tasks. As a prototypical example, medical language, specifically EHRs, may ignore many restrictions that are mandatory in standard grammar, such as the fact that each sentence must have a subject. Clinical texts are typically ungrammatical, which make the automatic temporal reasoning a difficult task outside the medical community.

In addition, temporal relation identification from clinical texts poses a special problem to NLP, as sentences in EHRs can be complex, including information about more than one medical event occurring at the same or at different time points. The determination of univocal relations between a date expression and the corresponding medical event can be very difficult.

Furthermore, another problem emerges from the instant-based representation of medical events. In real applications, it is difficult to relate all medical events to their exact occurrence timing. Free texts include diverse, complex, and sometimes non-standard linguistic mechanisms for mentioning temporal relations. In some cases, the time associated to a medical event is not even explicitly mentioned. 
Chapter 4. Reconstructing the Patient's Natural History from Electronic Health Records 4.2. Problem Statement

\subsubsection{Challenge 2 - Derivation of the Order of Medical Events from Patient's EHRs}

In order to generate a comprehensive medical timeline describing the patient's natural history, and thus exploit the temporal succession of medical events, it is firstly necessary to identify the temporal ordering of medical events across EHRs. As discussed in [178], this is a challenging problem in general NLP as well as the clinical domain, as the texts across patient's EHR lack logical continuity: the narrative goes back and forth in time, describing medical events that have happened at different time points.

In addition, in general linguistics, events are often expressed by verbs, and thus tense and aspect are the elements used to temporally order them. This is nevertheless not always true in the context here tackled, as many medical events are noun phrases [179], and most EHRs are written in past tense.

As a last point, it is important to highlight the problem of information redundancy, a fundamental concept associated with EHRs that arises both within and across clinical data sources. The same medical event can be mentioned in multiple EHRs, especially because of two reasons: the tendency to re-use past notes to save time, i.e. by copying and pasting part of a previous note; and the interest to summarize past information in the newly generated EHRs.

Two or more medical events are said to be similar (or also coreferential), if they have the same semantical category, value (if any) and have occurred on similar or consecutive time points. To illustrate, consider 4.1, in which the same medical event "Stage II" is mentioned four times on "04/01/2017", "05/01/2017", "15/06/2017"; these mentions are coreferential, i.e., refer to the same event, and it is then necessary to determine the exact and real occurrence time of the medical event. 
Chapter 4. Reconstructing the Patient's Natural History from Electronic Health Records 4.3. State of the Art

\subsection{State of the Art}

Events happen in time, and hence time is the main parameter for interpretation of event occurrences. To automatize the procedure of temporal reasoning and ordering of medical events extracted from multiple EHRs on a timeline, first, we review the literature.

We start our discussion with the definition of event by linguists and philosophers and how their definition differs from "Medical Event" in Section 4.3.1. Afterwards, we review time representation methods and the systems introduced for automatic temporal relation discovery from English and Spanish texts in Section 4.3.2. Finally, we provide the details of the systems and models introduced for temporal ordering of medical events across English written EHRs in Section 4.3.3.

\subsubsection{Definition of Event in General Domain VS Med- ical Domain}

Linguists and philosophers have long debated the meaning of event and define the event in different ways. In linguistics, an event is defined as a kind of information in discourse that actually occurs at now point on the timeline and is overt [180] whereas in philosophy, events are either objects in time or instantiations of properties in objects 181 .

However, in medical domain, events are temporally associated objects, describing medical conditions that affect the patient's clinical status or the procedure performed on a patient [182]. The main protagonist of a medical event is usually a patient. The structure of medical events, including states and incidents, could compose of a host of participants, props, locations and times 183. Medical events usually describe diseases and disorders, signs and symptoms, medications, laboratory results, as well as general events such as discussion of medications side effects, planning of procedures, etc. [184].

Syntactically in clinical texts, medical events are restricted to noun or noun phrases concepts [179]. For example, the concepts such as "Lung cancer", "Tumor stage II" or "Surgery" are considered as medical events in clinical texts. 
Chapter 4. Reconstructing the Patient's Natural History from Electronic Health Records 4.3. State of the Art

\subsubsection{Temporal Reasoning}

Time is an essential component of medical events. Time information is intrinsically connected with temporal reasoning that has a long-standing interest to biomedical community [168]. Studies of time-oriented applications have been performed in multiple clinical areas such as cardiology, oncology, psychiatry, internal medicine, intensive care, cardiac surgery, orthopedics, urology, infectious diseases, anesthesiology, pediatrics and endocrinology [185.

Back in 1970s, time-oriented databanks [186] dealt with storage and processing of explicitly timestamped clinical variables. Early expert medical systems such as: MYCIN [187] and ONCOCIN [188] discovered the necessity to associate time information with clinical data. However, these systems provided a fixed set of rules, which were disease specific in many cases and hence had limited ability for finding the temporal nature of clinical data for reasoning. These systems mostly dealt with limited categorical clinical variables such as: bilirubin levels, WBC count, blood pressure readings and etc.

1990s saw many research in temporal reasoning. However, most of them focused on the structured medical records stored in databases [189 192]. In this Section, firstly, we review the methods used for representation of time by temporal reasoning systems. Afterwards, we extensively review the literature on the most important systems introduced for temporal relation discovery from English clinical texts from 1980s onwards. Finally, we explain the related works for temporal reasoning from Spanish Free texts.

\section{Time Representation Methods by Temporal Reasoning Systems}

Time information are recorded in the free texts of EHRs [193]. In natural language, effective reasoning with temporal knowledge requires a proper representation of time. There are two methods of time representation, used by the temporal reasoning systems [179]:

1. Instant-based Representation: also called as Point-based Representation, which involves presentation of the exact time on which an event is occurred. In general, an instant-based representation is desirable if every event can be assigned a date. Unfortunately, in real applications, it is difficult to assign exact dates to all the medical events. 
Chapter 4. Reconstructing the Patient's Natural History from Electronic Health Records 4.3. State of the Art

2. Interval-based Representation: also called as Period-based Representation, which involves presentation of the duration (begin and end of time points) on which an event is occurred.

Early problem-solving systems designed to compute temporal relations from a structured input of time specifications and provided the instant-based representation of time 194,195]. In these systems, time points are the basis of temporal reasoning and time intervals can be constructed from these points.

Before the interval-based representation by Allen [196], there was not much prior work on computer interval-based representations of time. In the 1970s, Kahn and Gorry 197 presented a Time Specialist program, which accepts time relation in a LISP-like form as input. Then, the specialist program computes temporal relations, check for inconsistencies in the input and answers the questions about temporal relations between the events. This program supports several ways of organizing temporal relations to facilitate information retrieval.

Allen's time representation varies from that of Kahn and Gorry as it maintains relative temporal relationships in a highly structured fashion. It also includes a notion of the present time (i.e., now), which is provided in a manner that does not need knowledge of the exact present time [196]. Later on, researchers in [198 200] proposed using both instant-based and interval-based time representations with the same level of importance.

\section{Temporal Relation Discovery from English Clinical Texts}

Temporal Reasoning has been active area of research in the field of artificial intelligence and has many applications in the research areas of NLP, data mining, robotics and database design and query 201]. We limit the scope of this state-of-the-art to temporal reasoning in NLP with a focus on applications in clinical informatics.

The growing interest in NLP applications like question answering [202,203, clinical outcomes prediction [204] and the recognition of temporal patterns and timeline 205 places increasing demands on temporal information processing in natural language. Automatic temporal relation discovery of medical events 
Chapter 4. Reconstructing the Patient's Natural History from Electronic Health Records 4.3. State of the Art

that are appeared in clinical texts, will dramatically increase the understanding of medical phenomenas such as patient's clinical course, disease progression, and longitudinal effects of medications. In this Section, we review the most important systems developed for linking medical events to time expressions in English clinical texts from 1980s onwards.

In1980s, some studies were done on retrieving time information from natural language texts 206 in the Linguistic String Project (LSP) at New York University [207]. The LSP introduced one of the first NLP systems for English language [208 that was eventually adapted to clinical texts [209]. A Time program was developed to take the output of the NLP system, recognizes and analyzes time information, and formalizes various time expressions in a suitable representation.

In addition, this Time program was able to support the detection of medical events by assigning each medical event a time point or period. As a result, this program was capable of answering time-related quires such as: "Was a blood culture done at admission?".

In 1982, the authors of 210] identified words and structures that point to time information in discharge summaries. Such identifications embraced explicit temporal information like adverbial expressions of time and verb tense as well as implicit information like narrative time progression and multiple references to the same event in a text.

In the year 1986, an NLP system, named Grammatical Representation of Objective Knowledge (GROK) 211] was developed for analyzing temporal information in clinical texts using interval-based approach, where an event is defined as an entity of finite duration. To order and group events, the authors defined key events as domain-specific concepts.

The GROK system was able to perform the following tasks: (1) parsing a medical journal text by using linguistic and extra linguistic knowledge; (2) mapping the parsed linguistic structure to an event-representation; (3) drawing temporal and factual inferences in liver diseases domain; (4) creating and updating a database containing information about a patient. 
Chapter 4. Reconstructing the Patient's Natural History from Electronic Health Records 4.3. State of the Art

Modern statistical approaches and advances in NLP have enabled representations and learning over large-scale patient data in medical domain. However, creation of publicly accessible clinical corpora is costly, time consuming and most importantly, difficult due to the privacy concerns on medical data. The complex nature of the medical sub-language also makes it more difficult to annotate clinical texts with temporal information.

Despite of the aforementioned hurdles, the 21st century has seen a considerable amount of research in processing temporal information in clinical texts using rule-based methods and statistical machine learning techniques.

Until around the year 2003, there was no shared resources on automatically learning temporal relations from unstructured textual data. Since then, there have been efforts to generate a shared corpus of newswire text annotated with events, time expressions and temporal relations. As a result, a newswire corpus named TimeBank [150], was generated, containing 183 news articles.

The TimeBank corpus used the annotation guidelines of a specification language, called TimeML 149]. The TimeML is an emerging standard for annotation of events, time expressions, temporal relations and the ordering of events relative to one another in text.

The TimeML annotation schema denotes events, time expressions and temporal relations using the EVENT, TIMEX3 and TLINK tags. The EVENT tag is assigned to tensed verbs, stative adjectives and nominals. Whereas, the TIMEX3 tag is assigned to one of the following types [212]:

1. DATE (e.g., Friday October 1 1999, last week)

2. TIME (e.g., ten minutes to three, late last night)

3. DURATION (e.g., 2 months, 48 hours)

4. SET (e.g., twice a week, every 2 days)

Furthermore, the TLINK tag is used for representing temporal relations holding between events or between an event or a time. 
Chapter 4. Reconstructing the Patient's Natural History from Electronic Health Records 4.3. State of the Art

Using the TimeML schema, TimeBank corpus has evolved as a shared resource for learning temporal relations in the NLP community [213]. To evaluate systems that annotate texts with temporal information using TimeML and TimeBank corpus, TempEval [151,214 framework was introduced. The TempEval was developed in the context of the SemEval 2007 workshop for derivation of in-document timeline from news articles.

Although the TimeML group has developed a temporal annotation guideline, it only focuses on the news article domain. As mentioned in [143] "a significant theoretical foundation is a sub-language theory, which shows a language in a restricted domain is more well-defined than the general domain and can be more accurately characterized by specific vocabularies, semantic relations and in some cases, syntax". This statement implies that the TimeBank corpus is not suitable to be applied in the medical domain, as clearly a solution for news documents may not work properly on medical ones.

In recent years, the interest for temporal information identification from clinical texts has steadily been growing, partly due to the widespread adoption of EHR [33]. In 2011, an end-to-end system was developed to process clinical narratives written in English [215]. This system used rule-based approaches for the extraction of medical events, time expressions, temporal relations and the construction of medical timelines from individual clinical records.

In order to foster research activities on temporal relation discovery in the medical domain, the i2b2 NLP Challenge [163] was launched in 2012, providing an English corpus of discharge summaries annotated with events, time expressions and temporal information. Using this corpus, researchers were able to extract a limited set of temporal relations using rule-based and machine learning methods.

In the context of i2b2 NLP Challenge, the highest F1 score of 0.69 for the problem of temporal relation identification was achieved by two organizations: the Vanderbilt University on one hand, which proposed a rule-based pairwise selection with CRF and Support Vector Machine (SVM); and the National Research Council Canada, on the other hand, which implemented Maximum Entropy (ME), SVM and rule-based methods. 
Chapter 4. Reconstructing the Patient's Natural History from Electronic Health Records 4.3. State of the Art

A hybrid system was also designed for identification of temporal relations from clinical texts, which combined graph reasoning, SVM and rule-based classification in 2013 [216]. This system was validated using the test data set (120 clinical notes) of the 2012 i2b2 NLP challenge, obtaining an F1 measure of 0.63 .

In addition, a method was also presented in [217] to extract temporal relations from clinical texts. As its first step, this method built a feature set appropriately for clinical expressions, followed by the development of a semisupervised framework for temporal event extraction, and finally recognition of temporal relations among events with a formulated hypothesis. This method was evaluated on 2012 i2b2 dataset and gained the F1 score of $67.1 \%$ for temporal relation extraction.

The authors of [218,219] modeled the temporal information appeared in clinical discharge summaries, written in English as Simple Temporal Problem (STP), which is also formally referred to as Simple Temporal Constraint Stratification Problem (STCSP). Based upon this work, an architecture was proposed in 143 for representing, extracting and reasoning about temporal information in clinical narrative texts, which was then incorporated both in the MedLEE system [75] and in TimeText 220]. This latter system obtained the recall of $79 \%$ in identification of temporal relations from fourteen discharge summaries, which were obtained from the clinical data repository at Columbia University Medical Center.

The enabling technologies for temporal relation and timeline discovery from clinical narratives were evaluated in [173]. As a result, an extension of ISO-TimeML guidelines was developed for annotation of a corpus of clinical notes, which was written in English and was provided by the Mayo clinic, named THYME [168].

Many systems were developed for extracting events, time expressions and temporal relations using THYME in the context of Clinical TempEval 2015 164, Clinical TempEval 2016 [165 and Clinical TempEval 2017 [165]. These systems used rule-based and machine learning models (e.g., SVM, CRF, Recurrent Neural Network ( $(\mathrm{RNN})$, logistic regression, etc.) for their implementation. 
Chapter 4. Reconstructing the Patient's Natural History from Electronic Health Records 4.3. State of the Art

In Clinical TempEval 2015, the participated systems were BluLab [169], KPSCMI and UFPRSheffield [221]. However, BluLab was the only system presented for identifying temporal relations. It used features generated from cTAKES with $\mathrm{CRF}++$ methods for identifying relations between medical events and document creation time, called DocTimeRel (DR). For DR, BluLab reached an F1 score of 0.702 when the raw texts were used as input, and an F1 score of 0.791 when manually annotated events and time expressions were provided.

In addition, BluLab also used features from cTAKES with the integration of $\mathrm{CRF}++$ and rule-based techniques for the discovery of relations between medical events and/or time expressions, called Container Relation (CR). For CR tasks, when raw texts were provided as input, BluLab achieved an F1 score of 0.102 without temporal closure, and an F1 score of 0.123 with it. In addition, for CR with manually annotated events and time expressions as input, BluLab reached F1 scores of 0.143 (with temporal closure) and 0.181 (without temporal closure).

In Clinical TempEval 2016, the participated systems were brundlefly [222], CDE-IIITH [223], Cental [224], GUIR [225], HITACHI [226], KULeuvenLIIR [227], LIMSI 228], LIMSI-COT [229], ULISBOA [230], UtahBMI 231, UTA-MLNLP [232], UTHealth [170], VUACLTL [233].

In context of Clinical TempEval 2016, UTHealth submitted two runs for its implementations based on linear and structural (HMM) SVM, using lexical, morphological, syntactic, discourse, and word representation features. UTHealth run-1 was recognised as the best performing system, with F1 scores of 0.756 and 0.479 for DR and CR respectively, when plain texts were given as input. It also obtained the highest F1 score of 0.573 for $\mathrm{CR}$ when the manually annotated medical events and time expressions were provided as input.

However, for detection of DR when the input was given as annotated medical events and time expressions, UtahBMI gained the highest recall of 0.843. It implemented CRF and SVM models and used lexical, morphological, syntactic, shape, character pattern, character n-gram, section type, and gazetteer features. 
Chapter 4. Reconstructing the Patient's Natural History from Electronic Health Records 4.3. State of the Art

In context of Clinical TempEval 2017, the participated systems were GUIR [172], Hitachi [234], KULeuven-LIIR [171], LIMSI-COT [235], NTU-1 [236], ULISBOA 237 and XJNLP 238.

Among all the presented systems for Clinical TempEval 2017, LIMSICOT used a combination of RNN with character and word embeddings, and SVM models with words and PoS tags as features. It obtained the best F1 scores for both unsupervised and supervised domain adaptations. For DR, it achieved F1 scores of 0.60 and 0.66 for unsupervised and supervised domain adaptions, respectively. Furthermore, for CR, it obtained an F1 score of 0.40 for unsupervised domain adaption and the F1 score of 0.43 for supervised domain adaptions.

An extension of cTAKES [100] was also proposed, based on an open-source temporal relation discovery system, and evaluated on the THYME corpus used in Clinical TempEval 2015, and on the 2012 i2b2 corpus 174, 184. This system used multiple supervised machine learning models for extracting the document creation time and within-sentence temporal relations. It achieved F1 scores of 0.807 and 0.321 for DR and CR, respectively on the THYME corpus by using an SVM classifier. Also, it obtained an F1 score of 0.695 for the overall evaluation on all types of relations on the 2012 i2b2 corpus by implementing an SVM classifier and rules for coreference pairs.

In 2016, these authors presented an automated method to generate more high-quality training instances for temporal relation discovery in [112]. This method semantically expanded gold medical events based on UMLS using two within sentence temporal relation classification models. It included SVM as the learning algorithm for models.

One of the models was developed for the identification of temporal relations between medical events and time expressions, while the second was developed for the detection of temporal relations between medical events. With the presented method, their temporal relation discovery system was evaluated on the colon cancer set of the THYME corpus used in Clinical TempEval 2015 and Clinical TempEval 2016, and achieved the F1 score of 0.594. 
Chapter 4. Reconstructing the Patient's Natural History from Electronic Health Records 4.3. State of the Art

In 2018, it was claimed in 239 that despite of the considerable amount of research done for temporal relation identification in clinical texts, the state of art performance was not high enough for practical applications. As a result, an SVM-based system was developed for identifying direct temporal relations at sentence level in clinical notes, written in English.

This SVM-based system is composed of three parts: (1) a pre-processor, which performs tokenization, section identification, PoS tagging, dependency parsing and semantic role labeling; (2) an SVM classifier, which discovers direct temporal relation between an event and a time expression within a sentence; and (3) a post-processor, which uses deterministic rules to fix common errors emerging in the process. This system was evaluated on 310 discharge summaries and obtained an F1 score of 63.77.

In addition, a tree-based bidirectional Long Short Term Memory NetworkRNN end-to-end model proposed in [240 was adapted to extract intrasentential temporal relations from clinical texts [241]. This model was evaluated on the Clinical TempEval 2016 THYME corpus and obtained an F1 score of 0.629 for the identification of CR.

Furthermore, a method based on feature engineering was built over LIMSICOT system [229] for extraction of temporal relations from clinical notes [235]. This method was evaluated over THYME corpus and gained micro-average F1 scores of 0.87 and 0.93 for DR and CR tasks, respectively.

Also, a pipeline for data driven medical event detection from social media using minimal supervision was developed to generate the medical time-line of events [242]. This pipeline includes two components of medical event extractor and temporal resolver, which mainly uses a set of rules for its implementation. It was validated on two datasets of Ravelry 243 and user's post from an online breast cancer community [244], and achieved F measure of 66.73.

Most of the current state-of-art systems did not show an adequate level performance for temporal relation extraction from Clinical texts written in English. In addition, many of these systems implemented machine learning techniques for temporal reasoning tasks by using annotated corpora, provided 
Chapter 4. Reconstructing the Patient's Natural History from Electronic Health Records 4.3. State of the Art

by the shared tasks. However, the limited size of such corpora can unavoidably affect the quality of processing.

\section{Temporal Relation Discovery in Spanish Free Texts}

The TimeBank corpus was used in three temporal analysis evaluation tasks in the SemEval competitions i.e., TempEval-1 [151], TempEval-2 [152], and TempEval-3 [153] for temporal relation discovery from newswire texts.

While the Temp-Eval-1 provided the TimeBank corpus for English Language, the TempEval-2 provided this corpus for six languages, including English, Spanish, Italian, French, Chinese, and Korean. In TempEval- 2, TIPSem [31] used Conditional CRF models to recognize temporal relation from Spanish free texts. TIPSem achieved a precision of 0.81 in the identification of temporal Relations between events and time expressions, and a precision of 0.59 in the discovery of temporal relations between events and document creation time. Furthermore, while Temp-Eval-3 also provided the TimeBank corpus for Spanish, no systems were presented for finding temporal relations from newswire texts.

TIPSem is the only work presented for temporal relation discovery from Spanish newswire texts and to the best of our knowledge, no systems have been introduced for identification of temporal relations from Spanish clinical texts

\subsubsection{Temporal Ordering of Medical Events Across En- glish EHRs}

In 2018, the authors of [245] proposed two metrics of Mean Squared Error (MSE) and Pairwise Ordering Accuracy (POA) to temporally order medical events mentioned within each clinical note in a listwise fashion. They also used ListNet [246], which implements neural networks as a listwise temporal ranking model in order to generate the timeline of medical events from clinical notes. By testing their model on THYME corpus, they achieved the MSE of 0.072 and POA of 0.517 . 
Chapter 4. Reconstructing the Patient's Natural History from Electronic Health Records 4.3. State of the Art

Discourse structure, logical flow of sentences and context play a great role in ordering of medical events based on temporal relations. However, the cross-EHR temporal ordering is challenging. As Radev mentioned in [178], clusters of typically related documents, containing events, which are evolved over time, are a challenge for NLP.

In context of SemEval-2015 task 4 [247] on cross-document event ordering in general domain, a set of 4 teams submitted their results on the provided dataset by the task. WHUNLP team used the Stanford CoreNLP [248] and a set of rules for its implementations, and achieved the highest F1 score of $7.28 \%$ when the raw texts were provided as input. However, when the gold event mentions were available, GPLSIUA team by using OpeNER language analysis toolchain [249], the TIPSem tool and Semantic Role Labeller from SENNA [250] obtained the best F1 score of $25.36 \%$.

In addition, a timeline extraction approach was proposed in [251], which generated noisy training data to anchor events to entities and temporal expressions by using distant supervision. This approach was validated on SemEval-2015 task 4 dataset and obtained F1 score of 28.58 when the gold event mentions were available.

The rest of this Section focuses on presenting systems developed for ordering patient's medical events on a timeline, which are mentioned across EHRs written in English.

In 2012, an annotation schema was developed in the Ohio state university to extend the TimeML annotation guidelines to capture medical events from clinical texts [252]. The Medical Event Tag was assigned to events, which refereed to a word or contiguous group of words found in a clinical narrative and described a medical condition effecting the patient's health. In addition, a medical event was represented as a time duration with a corresponding start and end time. Using linear-chain CRF, each medical event was anchored to a coarse time-bin (e.g., before admission, on admission, after admission, etc.) 177.

Afterwards, the temporal ordering of medical events mentioned in a single clinical narrative, was implemented using SVM-rank 253 and based on the 
Chapter 4. Reconstructing the Patient's Natural History from Electronic Health Records 4.4. Solution

medical events proximity to the admission date 254]. Finally in 2014, a framework for aligning medical event sequences across clinical narratives was developed based on coreference and temporal relation information using cascaded Weight Finite-State Transducer (WFST) [255]. This framework was evaluated on a set of 7 patients (80 clinical narratives overall) and obtained an accuracy of $78.9 \%$.

Further in 2016, To generate a deep phenotype of individual cancer patients from English Clinical documents, a multi-scale information model, known as Deephe, was built on top of Apache cTAKES NLP system [113. The model generated the deep phenotype of individual cancer patients, where deep phenotype refers to a set of attributes representing the clinical expression of a disease over time. Deephe used cTAKES NLP system for extracting information from texts.

Although the aforementioned systems can be used for building medical timelines from patient's EHRs, they are English-centric and also, extraction of the evolution of medical events on a timeline remains an unsolved problem. Therefore, in line of this research, we propose an NLP framework, which is capable of extracting temporal relations from Spanish clinical texts and building the evolution of medical events mentioned across patient's EHRs on a timeline.

\subsection{Solution}

EHRs are rich clinical data sources, containing information about the patient's medical care. Therefore, we introduce an NLP framework to mine EHRs in order to build the patient's medical timeline. This framework accepts XMI files annotated with medical concepts and date expressions as input and yields the natural history of the patient using a medical timeline, which starts with the diagnosis event and includes the evolution of the patient's medical condition. 
Chapter 4. Reconstructing the Patient's Natural History from Electronic Health Records 4.4. Solution

The framework is composed of two components (Figure 4.2): (1) Temporal Reasoning System, which links medical events to their corresponding date expressions in the EHR; and (2) Timeline Constructor, which generates the patient's medical timeline.

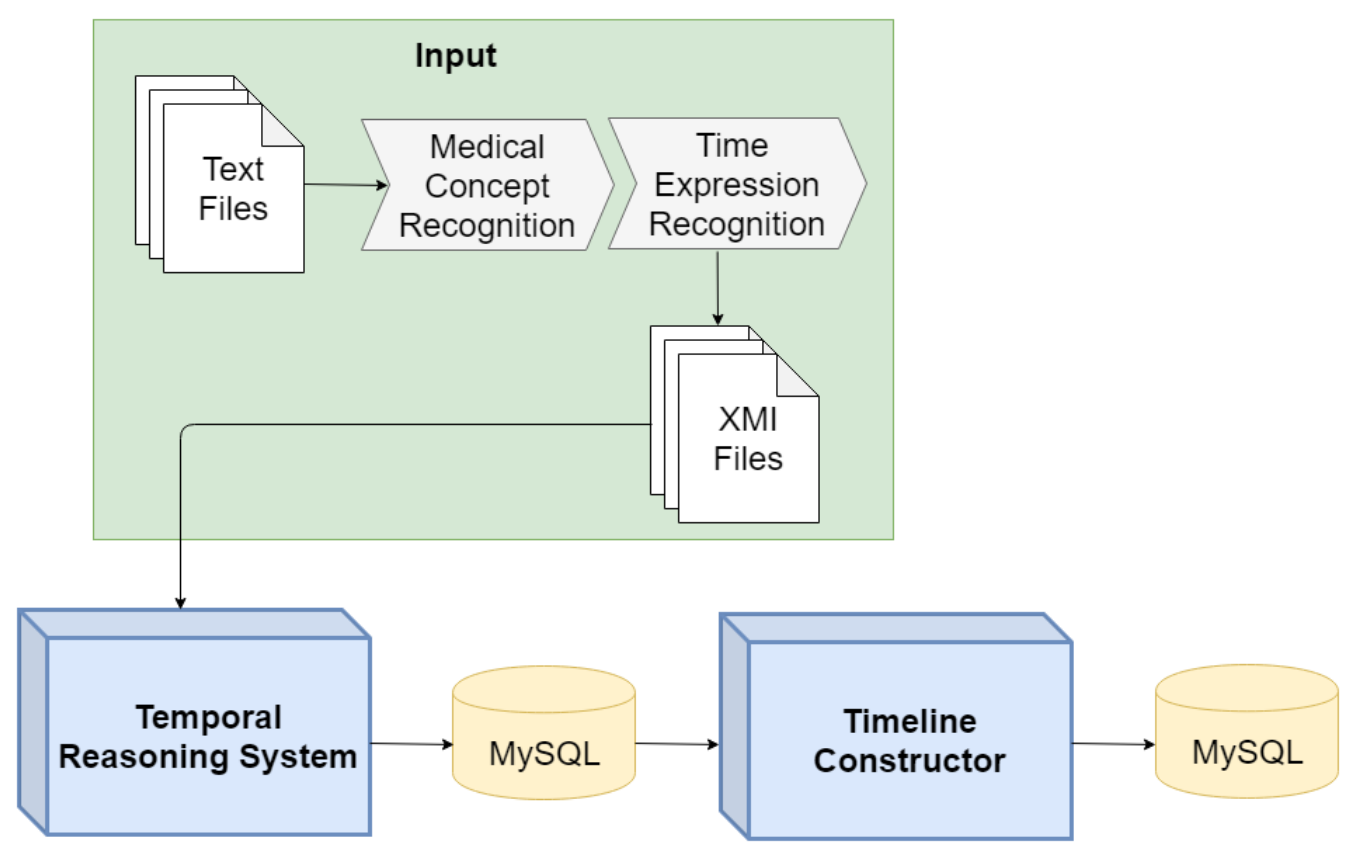

Figure 4.2: Architecture of the proposed framework

To better understand how our NLP framework builds the patient's medical timeline, consider the example of a patient's EHRs annotated with lung cancer diagnosis concepts, tumor stage codes and date expressions, which are provided in form of XMI files as input to our framework.

The Temporal Reasoning System reads these XMI files to identify the temporal relations between lung cancer diagnosis concepts and tumor stage codes with their corresponding occurrence date expressions. Once, all the temporal relations are extracted, they will be stored in a database. Afterwards, the Timeline Constructor reads this structured information and generates the patient's medical timeline, which starts with lung cancer diagnosis event and includes the evolution of tumor stage events. 
Chapter 4. Reconstructing the Patient's Natural History from Electronic Health Records 4.4. Solution

In the following Sections of 4.4.1 and 4.4.2, we provide the detailed information about the Temporal Reasoning System and Timeline Constructor of our NLP framework, respectively.

\subsubsection{Temporal Reasoning System}

To construct medical timelines, the medical concepts of interest and date expressions should be connected together, by finding temporal relations in the corresponding clinical texts at sentence, section or document level. In order to achieve this latter step, we developed a Temporal Reasoning System.

This system accepts as input XMI documents, containing the annotations of medical concepts and date expressions. Then, it identifies temporal relations by building the dependency parse trees using UDPipe [29, 30] tool and implementing a rule-based approach. Finally, once temporal relations are identified, the Temporal Reasoning System stores them into a MySQL structured relational database, named Document.

The following Sections describe the details of UDPipe for building the dependency parse trees and explain the details of four rules implemented in order to identify temporal relations from clinical texts of EHRs.

\section{UDPipe}

UDPipe is an open-source NLP tool, containing a pipeline of components such as tokenization, PoS tagging and universal dependency parsing for processing raw texts in multiple languages including Spanish. To generate the dependency parse trees, UDPipe uses a fast transition-based neural dependency parser, composed by a simple neural network with just one hidden layer and without any recurrent connections, and using locally normalized scores. The dependency parser builds an individual parse tree for each individual sentence in the clinical texts.

UDPipe employs nearly unmodified Parsito parser [256] for building dependency parse trees. Parsito is a transition-based and non-projective dependency parser, which parses both non-projective and projective sentences. This parser uses a neural network classifier to perform prediction and does not require feature engineering. It contains a search-based oracle that improves the 
Chapter 4. Reconstructing the Patient's Natural History from Electronic Health Records 4.4. Solution

accuracy of parsing as dynamic oracle, but it can be applied to any transition systems (e.g., fully non-projective swap system). To improve the accuracy of Parsito, UDpipe added an optional beam-search decoding.

To generate a dependency parse tree for a sentence, firstly, UDpipe accepts the texts as input and then, it assigns PoS (e.g., DET, NOUN, VERB, etc.) and dependency relation (e.g., det, nsubj:pass) tags to the tokens. Afterwards, using these tags, it determines the root node of the dependency tree and generates its child nodes.

For example, consider Figure 4.3, where the input sentence to UDPipe is "The patient is diagnosed with lung cancer on 16/09/2011.". After assigning the PoS and relation dependency tags to each word in the sentence, UDPipe selects the token of "diagnosed" as the root node. It then moves to the next level of the tree, where the child nodes are "patient", "is", "cancer", "16/09/2011", and ".". Afterwards, it generates level 2 of the tree with child nodes of "the", "with", "lung", and "on".

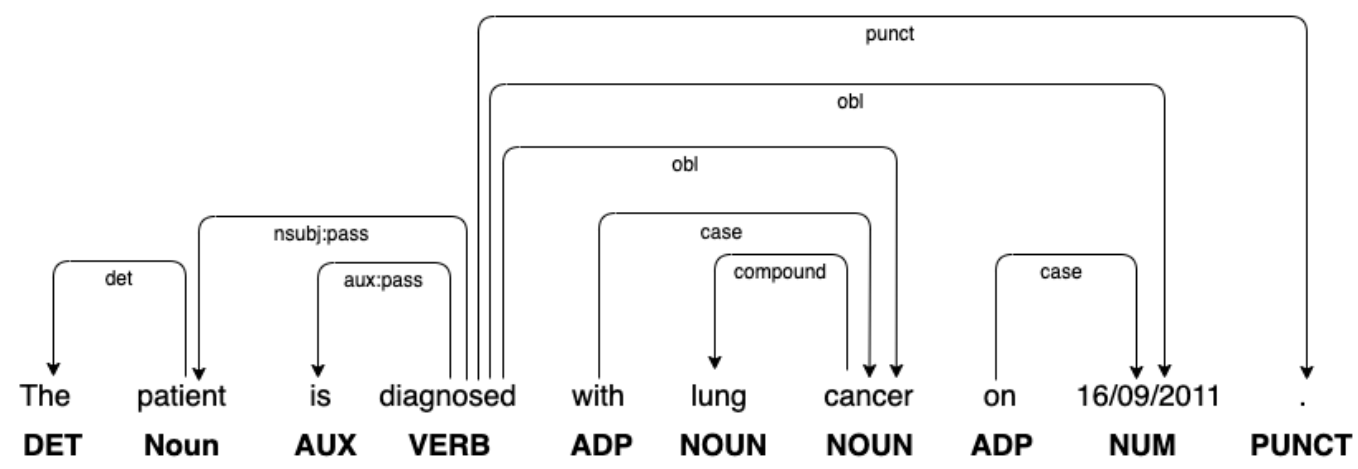

Figure 4.3: Example of dependency parsing by UDpipe

\section{Temporal Relation Identification}

Our objective is to unequivocally identify the date expression of each medical event. For this purpose, we define a temporal relation as:

1. A relation whose date expression modifies the medical event mention at the sentence level. This implies a syntactic construction where a medical event mention is directly accompanied by a date expression. 
Chapter 4. Reconstructing the Patient's Natural History from Electronic Health Records 4.4. Solution

2. A relation whose date expression and medical event mention are arguments of the same predicate at the sentence level. When both elements are arguments of the same predicate, they are considered to be temporally related. A predicate is usually defined in linguistics as a verb or a noun. A predicate requires one or more arguments in different syntactic rules to complete its meaning. Adjunct is another type of grammatical components, which modify or complete the meaning of a predicate. However, opposed to arguments, we can remove adjunct from the sentence without making it grammatically wrong.

3. A relation whose section date expression explains the occurrence timing of a medical event mention. Due to clinician's time limitations, it is a common practice to write patient information without specifically mentioning the date expression for each and every medical event in the same sentence; instead, this information is provided as a date in the section headings.

4. A relation whose document creation date expression determines the occurrence timing of a medical event mention. Medical events happen in time, i.e. they are temporally associated objects. To keep record of these events, clinicians write them in EHRs during each patient's visit in the hospital. Since clinicians usually have limited time for writing the patient-clinician encounter, they usually mention the medical processes followed or the conditions discovered on the same day of the patient's visit without a mention of a date expression at sentence or section level. At the coarsest level, medical events are temporally related to the EHR creation date.

Based on the above definitions, the Temporal Reasoning System follows four rules to link medical event mentions to the date expressions (examples are provided in Table 4.1 to identify the occurrence date of the medical event "lung cancer"):

- Rule - 1: If at sentence level, a medical event mention is an ancestor of a date expression in the dependency path or vice versa i.e., if a date expression is an ancestor of a medical event in the dependency path, they form a temporal relation based on syntactic structure, and therefore the medical event mention should be linked to that date expression. Otherwise, move to the next rule. 
Chapter 4. Reconstructing the Patient's Natural History from Electronic Health Records 4.4. Solution

- Rule - 2: If at sentence level, the medical event mention and the date expression are arguments or adjunct of the same predicate i.e., if the medical event mention and the date expression have the same parent node of type noun or verb in the dependency path, they form a temporal relation based on predicate argument structure, and therefore the medical event mention should be linked to that date expression. Otherwise, move to the next rule.

- Rule - 3: If the section in which the medical event mention appeared contains a date expression in heading, they form a temporal relation, and should hence be linked. Otherwise, move to the next rule.

- Rule - 4: If a medical event mention appeared in an EHR, it forms a temporal relation with the document creation date and should be linked to it.

Table 4.1: Examples for rules of temporal relation identification

\begin{tabular}{|c|c|c|}
\hline Rules & $\begin{array}{l}\text { An Example that satisfies } \\
\text { the rule }\end{array}$ & $\begin{array}{l}\text { An Example that does not } \\
\text { satisfy the rule }\end{array}$ \\
\hline 1 & $\begin{array}{l}\text { Clinical Judgment: lung cancer } \\
\text { on 16/09/2011. - See Figure } 4.4\end{array}$ & $\begin{array}{l}\text { The patient was diagnosed with } \\
\text { lung cancer (stage IIB) and had a } \\
\text { surgery on 23/12/2016. - See } \\
\text { Figure } 4.5\end{array}$ \\
\hline 2 & $\begin{array}{l}\text { The patient is diagnosed with } \\
\text { lung cancer on 16/09/2011. - See } \\
\text { Figure } 4.6\end{array}$ & $\begin{array}{l}\text { The patient has had the lung } \\
\text { cancer (stage IIB) and a surgery } \\
\text { on 23/12/2016. - See Figure } 4.7\end{array}$ \\
\hline 3 & $\begin{array}{l}\text { Clinical Judgment - 16/09/2011: } \\
\text { Lung cancer, stage IIB. }\end{array}$ & $\begin{array}{l}\text { Treatment - 23/12/2016: } \\
\text { The patient had a surgery due to } \\
\text { diagnosis of lung cancer on } \\
\text { 16/09/2011. }\end{array}$ \\
\hline 4 & $\begin{array}{l}\text { Document Creation Date: } \\
\text { 16/09/2011 } \\
\text { The } 50 \text { years old patient is } \\
\text { diagnosed with lung cancer and } \\
\text { stage IIB. }\end{array}$ & $\begin{array}{l}\text { Document Creation Date: } \\
\text { 23/12/2016 } \\
\text { The patient had a surgery due to } \\
\text { diagnosis of lung cancer on } \\
\text { 16/09/2011. }\end{array}$ \\
\hline
\end{tabular}


Chapter 4. Reconstructing the Patient's Natural History from Electronic Health Records 4.4. Solution

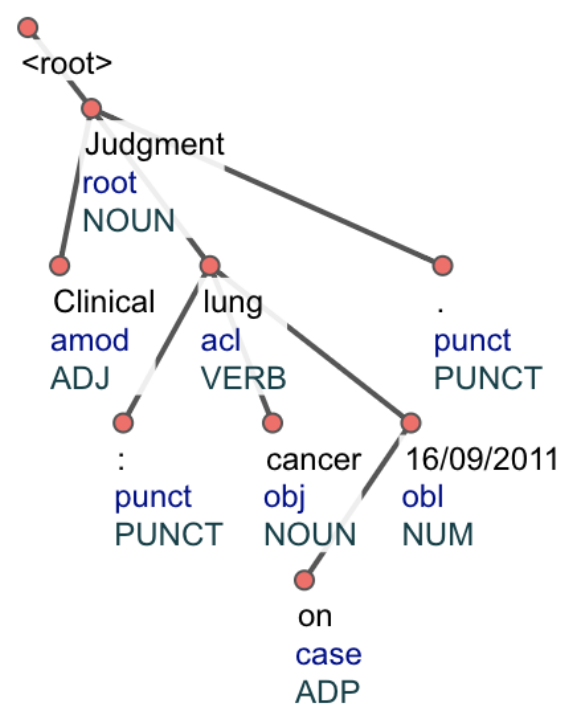

Figure 4.4: Dependency parse tree analysis for an example that satisfies Rule $1[6$

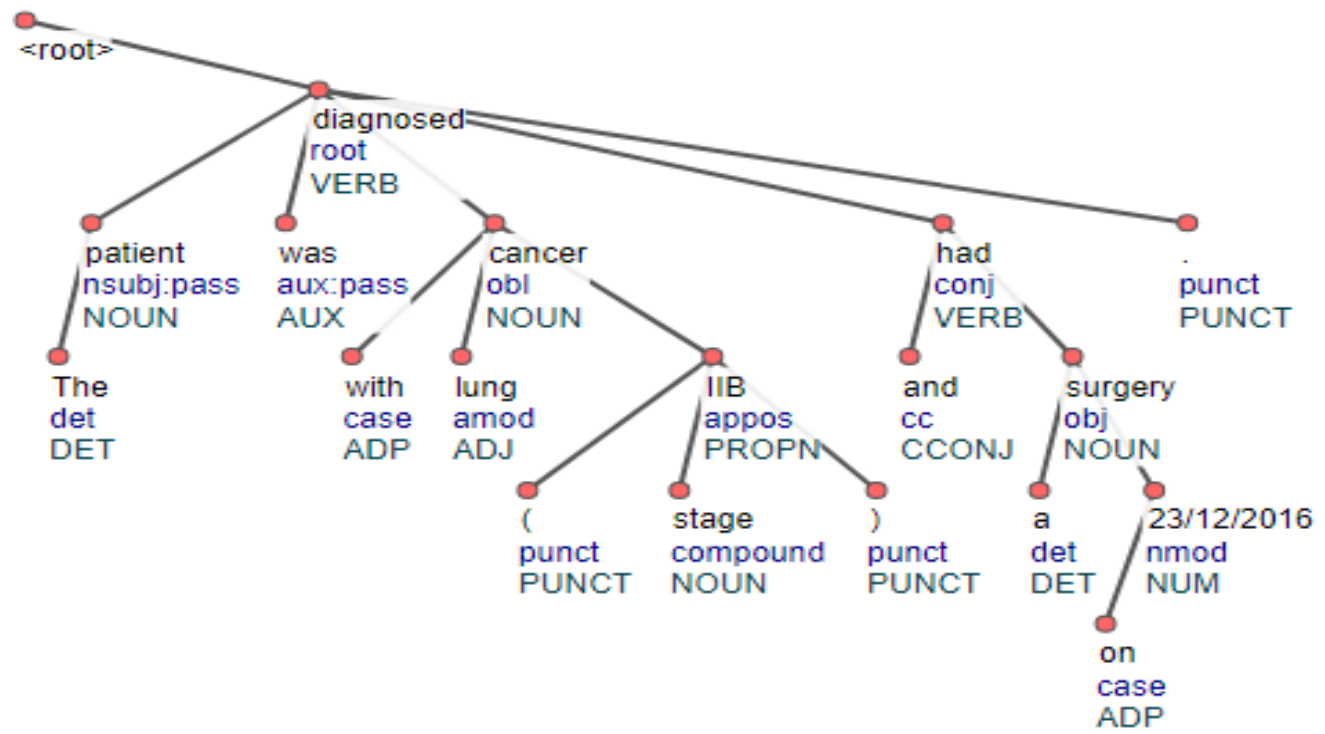

Figure 4.5: Dependency parse tree analysis for an example that does not satisfy Rule -16 
Chapter 4. Reconstructing the Patient's Natural History from Electronic Health Records 4.4. Solution

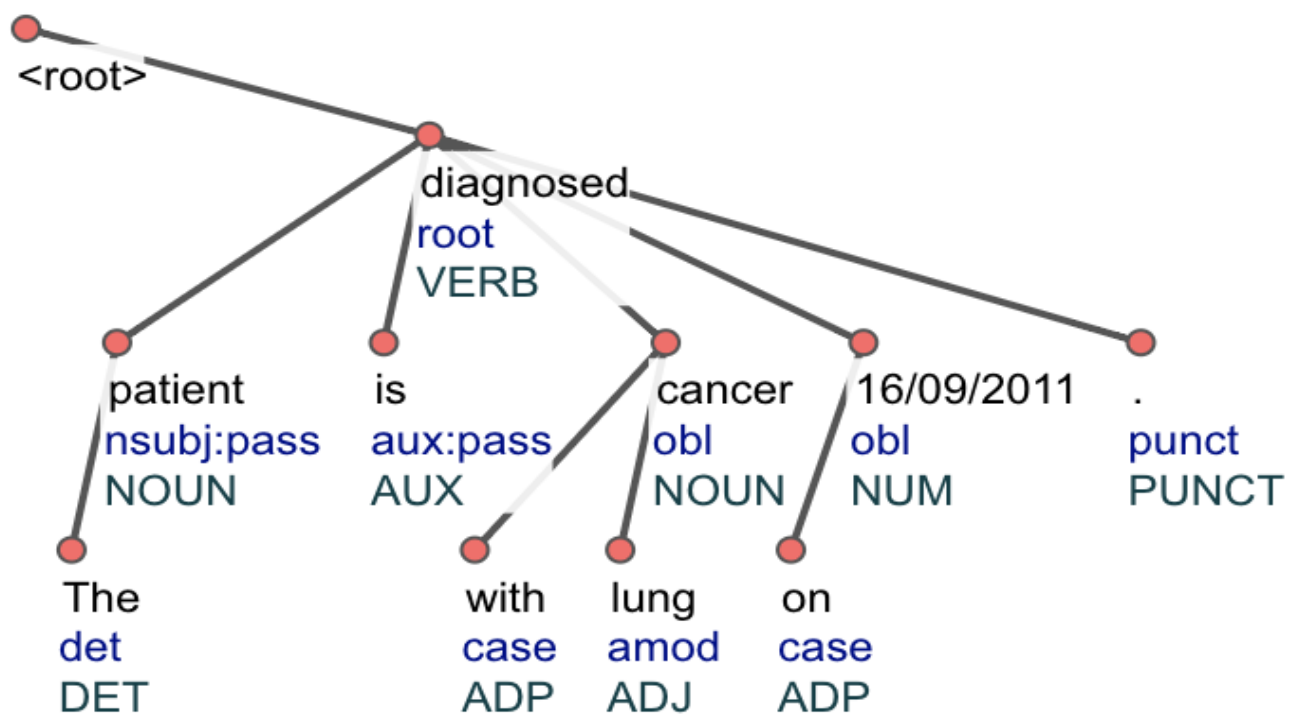

Figure 4.6: Dependency parse tree analysis for an example that satisfies Rule 26

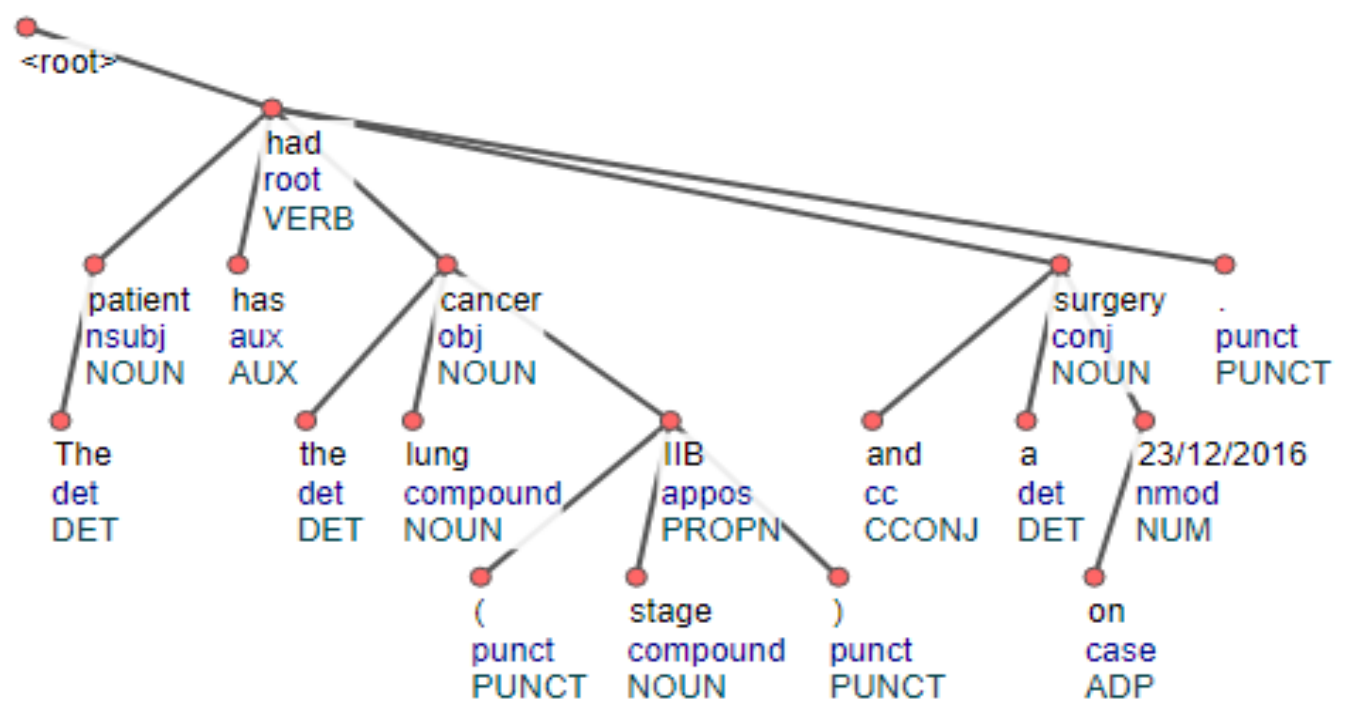

Figure 4.7: Dependency parse tree analysis for an example that does not satisfy Rule -26 
Chapter 4. Reconstructing the Patient's Natural History from Electronic Health Records 4.4. Solution

\subsubsection{Timeline Constructor}

To approach the problem of dealing with redundant medical events across patient's EHRs and ordering them into a medical timeline, we developed a component named Timeline Constructor. It accepts as input the structured information of Document database. It then processes this information to produce the natural history of the patient on a medical timeline, which starts with the diagnosis event and includes the evolution of patient's clinical condition. Finally, the timeline is stored into a relational database.

As mentioned in Section 4.2.2, when two or more instances of medical events have similar semantical category and value (in case of medical events that are metrics e.g., tumor "Stage IIB" is a metric having value "IIB") and have occurred on the same or consecutive time points, they are said to be coreferential. To identify medical events, which are coreferential across a patient's EHRs, a specific time range (e.g., difference of one month) for defining consecutive time points is not considered rather a combination of similarity in terms of semantical category and value on the same time points or those occurred one after another until the value is changed, is considered.

To determine whether two or more medical events are coreferential (See Listing 4.1), firstly, the Timeline Constructor temporally orders these events, for then evaluating their semantic similarity. Since it accepts the structured information of the Document database as input, it does not need to explicitly create complex procedures to discover semantic similarities between medical events. All the medical events with the same semantic category and with-

/ without different notation of words or continuous groups of words are stored within the same table in the Document database.

Consider the example of the lung cancer diagnosis and the tumor stage events, which are provided as structured information to the Timeline Constructor. Since this structured information is fed as two separate tables to the Timeline Constructor, this component can identify all the medical events belonging to the lung cancer diagnosis table are semantically similar to each other and semantically dissimilar to the tumor stage events. 
Chapter 4. Reconstructing the Patient's Natural History from Electronic Health Records 4.4. Solution

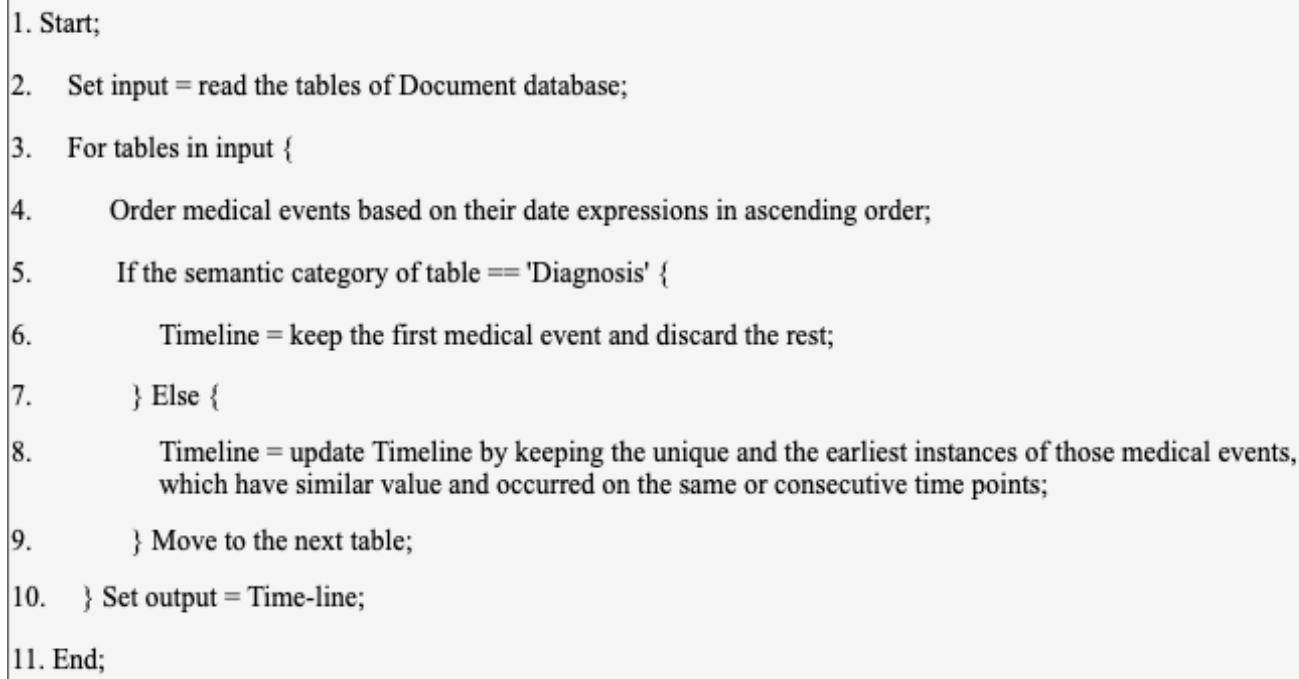

Listing 4.1: Pseudocode of Time-line Constructor

Secondly, if the medical event is a metric, value similarity is the second factor to be considered. Therefore, at this stage, decisions made by the Timeline Constructor are binary, meaning either multiple instances of medical events from the same semantic category have similar values or not. Consider Figure 1 as example where tumor stage events are "Stage II - 04/01/2017", "Stage II - 05/01/2017", "Stage II - 05/01/2017", "Stage II - 15/06/2017" and "Stage $I-05 / 07 / 2017$ ". In here, the first four tumor stages, have value similarity as they hold II value.

Thirdly, in order for medical events to be coreferential, the last factor considered is the occurrence time point. Therefore, the Timeline Constructor determines whether two or more medical events with similar semantic and value are overlapped or occurred on consecutive time points. For example, in Figure 4.1, the same medical event "Stage II" occurred four times on "04/01/2017", "05/01/2017", "15/06/2017"; these mentions are coreferential, i.e. refer to the same event.

Finally, to generate the timeline of the patient's medical care, the Timeline Constructor selects the diagnosis event with the earliest time point and discards the rest. Then, for the rest of medical events, the Timeline Constructor 
Chapter 4. Reconstructing the Patient's Natural History from Electronic Health Records 4.5. Validation

keeps the unique and the earliest instance of the same medical event on the timeline, and discards the rest of redundant medical events, as they do not introduce any change of state.

\subsection{Validation}

We here focus on validating our framework to reconstruct the natural history of patients, who suffer from lung cancer. To evaluate our framework, we used the dataset mentioned in Section 2.5.1.

From these EHRs, clinicians are interested in finding specific patterns in long surviving lung cancer patients. The identification of these patterns will help them to detect unknown associations of family history, treatments, response to treatments, toxicities, comorbidities and molecular mechanisms, with the patient's outcome. In order to identify long surviving patients, the detection of the date of the lung cancer diagnosis and the evolution of tumor stage codes are the key factors.

Therefore, we here evaluate our framework by building the patient's medical timelines, starting with the lung cancer diagnosis event and include the evolution of tumor stage events. However, the first step toward generating such a timeline from multiple EHRs is to encode, structure and extract lung cancer diagnosis and tumor stage concepts along with the date expressions from clinical texts, as these represent the basis of medical events. This is where the need for the NLP annotators comes into play. The input to these annotators is the plain texts of EHRs and the output is formatted as a set of XMI files containing annotation results.

To identify the diagnosis concepts, we use the rule-based UMLS Annotator of C-liKES [22], which is built upon UIMA framework. This annotator is designed to annotate noun and noun phrase concepts found in clinical texts that have contextually relevant matches in the UMLS as medical concepts. Example of such concepts are "Lung cancer", "Cancer of lung", "Ca lung", etc. 
Chapter 4. Reconstructing the Patient's Natural History from Electronic Health Records 4.5. Validation

To recognize tumor stage codes of lung cancer from clinical texts, we use Stage Annotator and the TNM Annotator presented in Section 2.4.2. These annotators are pattern-based extraction NLP modules built over UIMA framework and are capable of identifying named entities of stage grouping and TNM codes.

To extract and normalize time variables appeared in clinical texts, we use a rule-based NLP annotator built over UIMA framework, named Temporal Tagger, which is presented in Section 3.4. The Temporal Tagger is capable of: (1) extracting various time expressions, i.e., natural, conventional and professional time variables, written in different styles and format; (2) filtration of time expressions that are not likely to be time using their PoS tags; (3) resolution of time expressions with respect to the section or document date; and (4) normalization of time expressions to a standard date format.

Finally, the evaluation of our developed framework consists of two use cases:

1. Validation of the Output of the Temporal Reasoning System

2. Validation of the Output of the Timeline Constructor

This Section is organized as follows. Firstly, Section 4.5.1 explains the process of sample selection for first use case along with the details of statistical tests performed. Afterwards, Section 4.5.2 discusses the experiments conducted and finally, Section 4.5.3 presents their results.

\subsubsection{Sample Dataset Selection for First Use Case}

Due to the large pool of EHRs, performing the manual validation on the entire dataset was not feasible for the first use case. Therefore, to conduct our experiments, we have decided to randomly selected 200 temporal relations generated by our Temporal Reasoning System from 200 EHRs, including 100 clinical notes and 100 clinical reports. The selection of equal number of clinical notes and reports was aimed at keeping both types of documents equally represented in the validation process. 
Chapter 4. Reconstructing the Patient's Natural History from Electronic Health Records 4.5. Validation

Finally, a set of chi-squared statistical tests was also performed on the selected samples, to assess their representativeness of the entire population in the original dataset. These tests were performed on four significant variables:

1. Patient's sex

2. Patient's age (categorical variable: <35, 35-45, 45-55, 55-65, 65-75, $75-80,80>)$

3. Local progression of the tumor

4. Systemic progression of the tumor

Finally, the selected sample dataset was representative of the entire population ( $\mathrm{p}$-value $<0.01$ ).

\subsubsection{Experiments}

For the first evaluation task, two computer scientists served as the evaluation domain experts under the supervision of clinicians from HUPHM. They were native Spanish speakers and participated neither in the design nor in the development of the NLP framework. For the second evaluation task, four clinicians from HUPHM conducted the experiments. The details of these evaluation tasks are discussed in the following two Sections.

\section{First Use Case - Validation of the Output of the Temporal Rea- soning System}

The evaluation of our Temporal Reasoning System in processing EHRs involved the verification of its output temporal constraints. For each temporal relation generated by our Temporal Reasoning System, the verification was done by manually analyzing each EHR for the corresponding temporal relation in order to compute $\mathrm{TP}$ and $\mathrm{FP}$ for determining the precision.

Furthermore, as part of this task, we compared our Temporal Reasoning System with the Spanish version of TIPSem for identifying temporal relations from texts based on their precision. This comparison was done using the Spanish test dataset of TempEval-2 and based on its Task C, which focuses on the determination of temporal relations between an event and a time 
Chapter 4. Reconstructing the Patient's Natural History from Electronic Health Records 4.5. Validation

expression mentioned in the same sentence. This task is limited by requiring that either the event dominates the time expression syntactically or the event and time expression are mentioned in the same noun phrase.

\section{Second Use Case - Validation of the Output of the Timeline Con- structor}

The idea of our second evaluation task is to measure the precision of our Timeline Constructor in generating the timelines of medical events. The validation process was performed by manually studying all the EHRs for every patient and extracting the corresponding timeline, such that its starting point is the diagnosis, followed by the evolution of the tumor stage events.

A comparison was done between the timeline manually extracted by the evaluation domain experts and the timeline generated by our Timeline Constructor. An error was counted for the Timeline Constructor when: (1) it generated a timeline with a different number of medical events (each associated to a date expression) compared to the one extracted by the evaluation domain experts; and (2) it generated medical events with different date expressions for the same medical event identified by the evaluation domain experts.

\subsubsection{Results}

The results of the first evaluation task show that from the sample of 200 temporal relations, our Temporal Reasoning System correctly identified 178 temporal relations, being wrong in 22 instances, which correspond to a precision of 0.89 . In this sample, 63,51 and 86 of the temporal relations were related to the sentence, section and document creation dates, respectively.

In order to understand the nature of the detected errors, we analyzed the obtained results to determine which kinds of temporal relations are difficult to be detected through our Temporal Reasoning System. It revealed that identifying temporal relations at sentence level is the most complex one, leading to 12 errors, which were the consequence of:

1. Usage of very complex sentences and ambiguous temporal relations, as for instance, "Lung cancer cT2N3M1b stage IV, due to carcinoma- 
Chapter 4. Reconstructing the Patient's Natural History from Electronic Health Records 4.5. Validation

tous lymphangitis, EGFR mutated (L858R of exón 21), diagnosed in November 2014", which led to 5 errors.

2. Missing usage of the dot "." or new lines at the end of a sentence, which led to the mixing of two or more sentences and resulted in 6 errors.

3. Missing annotation of date expressions by Temporal Tagger, which led to 1 error.

In addition, 10 errors occurred due to the identification of section level temporal relations, where the medical events were actually related to the date expressions in the previous sentences (4 observations) or the document creation date (6 observations).

Also, as it can be seen in Figure 4.8, our Temporal Reasoning System achieved a precision of 0.928 for task $\mathrm{C}$ of TempEval-2, which outperformed TIPSem with a precision of 0.81 . Since, the sentences in TempEval-2 dataset are grammatically more accurate than the clinical texts, our Temporal Reasoning system achieved a higher precision on this dataset compared to the clinical dataset provided by HUPHM.

The results generated from the second evaluation task showed that the Timeline Constructor correctly extracted the complete medical timeline for 843 patients, while failing in 146 instances, which indicates the achievement of precision 0.852 . We have seen that a typical patient's timeline contains 3 entries, including one diagnosis and two tumor stage events with a usual temporal range of 84 months.

To find the nature of errors occurred, we analyzed the output extensively. We realized that the major reason was related to the incorrect temporal relations fed as input to the Timeline Constructor. However, we have seen that these errors are not catastrophic as they affect only parts of the timelines of patients. For instance, in the clinical text "Treatment-27 May 2014: A 48-year-old woman with lung cancer (Stage IIIA), will be treated with superior lobectomy and 4 cycles of adjuvant QT.", the Temporal Reasoning System inaccurately assigned the section date "27 May 2014" to the event "Stage IIIA", while this stage should have been linked to the document creation time "2014-05-27". While this error caused the event "Stage IIIA" to be placed 12 
Chapter 4. Reconstructing the Patient's Natural History from Electronic Health Records 4.5. Validation

\section{1}

0.95

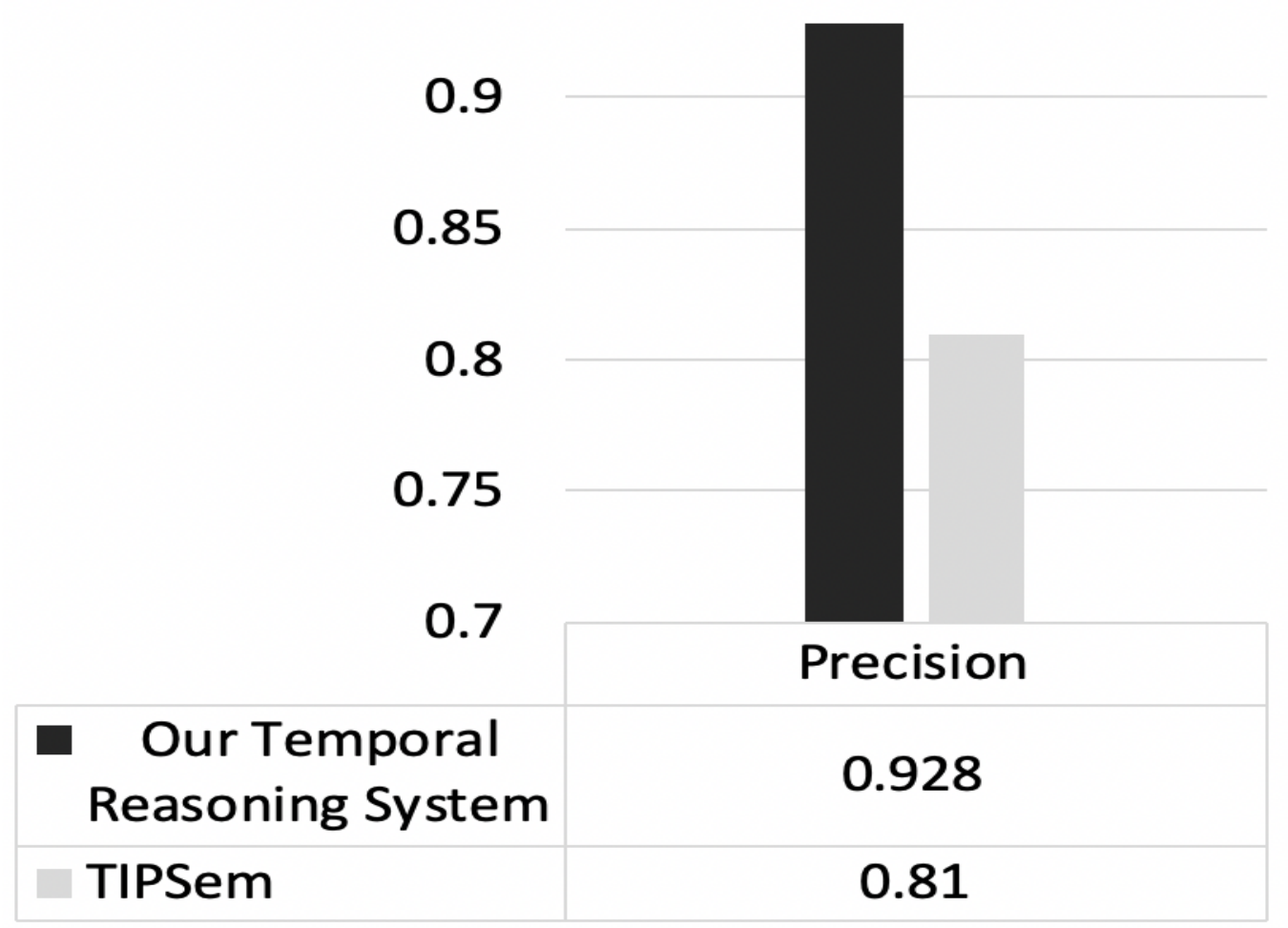

Figure 4.8: Comparison of the results of our Temporal Reasoning System with TIPSem

days later than its actual date on the timeline, it did not lead to any errors in relation to the diagnosis event and the rest of stage events. In addition, a few errors have been observed due to the limitation of our framework to detect negations and probabilistic terms (e.g., "likely to have lung cancer", "can be excluded from having lung cancer"). 
Chapter 4. Reconstructing the Patient's Natural History from Electronic Health Records 4.6. Discussion

\subsection{Discussion}

NLP technologies are helping researchers to extract new insights from large clinical and molecular datasets. Methodological pitfalls notwithstanding, NLP techniques are already beginning to affect cancer research and clinical care, such as early diagnosis and prevention [257], drug discovery [258], matching patients to clinical trials and treatment decisions [259].

In an era where data are being generated at an enormous pace - by 2020, it is expected that clinical data will double every 3 months, and that the average person will generate more than 1 million gigabytes of health-related data in their lifetime - it is increasingly difficult for clinicians to process all the available information that could influence treatment decisions. Furthermore, traditional analytics and machine technology have limited capability to utilize large complex datasets such as the so-called 'big data', and a change of paradigm is needed today to make the most from these potential sources of information. A major challenge is determining how to extract valuable information from the enormous amount of data available in EHRs, and research is ongoing to determine the best methodology to analyze data and reduce/eliminate unhelpful noise [14].

In this Chapter, our aim was to reconstruct the natural history of patients from EHRs, written in Spanish, using rule-based approaches. The alternative, i.e. the adaption of machine learning approaches, requires annotated corpora, whose construction is costly and time-consuming. Also, their small size can significantly affect the processing quality.

We have here shown that the rule-based approach is a viable alternative, which can yield very good results while avoiding the aforementioned problems. However, unlike rule-based approaches, machine learning techniques have learnability capability, and able to generalize and deal with new cases. Therefore, by obtaining the gold standard annotations to evaluate the performance of two tasks (temporal relations and the order of medical events), it is now possible to use these annotated EHRs to create a Spanish clinical corpus to be used by machine learning models. 
Chapter 4. Reconstructing the Patient's Natural History from Electronic Health Records 4.6. Discussion

Reasoning with temporal information in texts is crucial to the field of NLP and biomedical informatics. In this Chapter, we proposed a Temporal Reasoning System for extracting temporal relations from Spanish clinical texts using dependency parsing and a set of four rules. We found that most of the temporal relations generated by the Temporal Reasoning System are correct. We have also seen a few errors due to the presence of very complex sentences, usage of ambiguous temporal relations, missing of end of sentence indicators, and the limitation of our system to identify temporal relations that span over a single sentence. We have also observed that our Temporal Reasoning System achieved a better performance on task C of TempEval-2 compared to the Spanish version of TIPSem.

In addition, extracting the evolution of medical events from a patient's EHRs helps in improving the quality of healthcare and treatments. By implementing a rule-based approach, we have generated medical timelines, containing the evolution of patient's medical conditions. many of the timelines generated by our Timeline Constructor were accurate. A few errors were observed due to feeding incorrect temporal relations into the Timeline Constructor. However, these errors only effected some parts of the patient's timelines. In addition, the behavior of the Timeline Constructor could be improved by annotating negations and probabilistic terms.

Finally, although our NLP framework has been developed general enough so that it can be used with no further adaptations in every medical domain to extract temporal relations and generate medical timelines form EHRs written in Spanish, its use in other languages would require the translation of the developed rules. 


\section{Implementation and Deployment}

This Chapter focuses on the implementation and the deployment details of our NLP framework, which is capable of generating the medical timeline for lung cancer patients from their EHRs. In addition, to present the application of this technology, we also discuss an example of its utilization in a web platform, named iASiS, for performing QA process and calculating the survival time of patients.

\subsection{Introduction}

As mentioned in Chapter 1, the main goal of this Thesis is to be able to reconstruct the patients natural histories from their EHRs with focus on those, who suffer from lung cancer. To achieve the above mentioned goal, the processes of medical concepts recognition, time expressions extraction and temporal relations identification have been addressed in great details in the previous Chapters.

However, as mentioned in Chapter 4, in order to generate an evolutionary timeline of the patient's medical events, the exploitation of temporal order of these events is essential. Although, this problem has been addressed by the Timeline Constructor in the Section 4.4.2, the deep analysis of this problem and its implementation details are still needed to be discussed for the sake 
Chapter 5. Implementation and Deployment

5.2. Identifying the Temporal Order of Medical Events Across EHRs

of completeness. In addition, the technical details of our framework and its application in real world are also worth highlighting.

The rest of Chapter is organized as follows: Section 5.2 analyzes the problem of discovering the order of medical events from EHRs and discusses the implementation details of Timeline Constructor. Afterwards, Section 5.3 explains the technologies used for deployment of our framework. Then, Section 5.4 presents a real world example of our framework utilization for QA process and patient's survival time calculation. Finally, Section 5.5 draws some conclusions.

\subsection{Identifying the Temporal Order of Medi- cal Events Across EHRs}

The aim here is to analyze the problem of automatically discovering the order of medical events from EHRs in Section 5.2.1 and to discuss the implementation details of the Timeline Constructor in Section 5.2.2.

\subsubsection{Problem Analysis}

Once, the medical concepts, time expressions and temporal relations are extracted from EHRs and are stored into MySQ relational database of Document, the patient's natural history would be still locked in this structured information as it is presented in the Figure 5.1- contains diagnosis events, Figure 5.2 - stage grouping events, Figure 5.3- TNM events and Figure 5.4patients and EHRs information.

As shown in Figure 5.4, for the same patient, several clinical notes and reports can be generated along time. Each of these can contain new information obtained after consultation and possibly some repeated information to summarize the past situation of the patient up to that moment. For example, consider Figure 5.2 as an example. The values of tumor stage "II" and "III" have been repeated in EHRs for the same patient over time. This is one of the first challenges that needed to be tackled, as it is necessary to remove 
Chapter 5. Implementation and Deployment

5.2. Identifying the Temporal Order of Medical Events Across EHRs

\begin{tabular}{|l|l|l|l|l|l|l|lll}
\hline document & section & paragraph & sentence & id & begin & end & concept & cui & date \\
\hline 2356 & 3 & 1 & 0 & 13456 & 108 & 132 & adenocarcinoma de oulmon & C0152013 & $2015-01-06$ \\
11 & 0 & 2 & 3 & 13457 & 257 & 273 & cancer de Dulmon & C0242379 & $2015-01-06$ \\
1238 & 0 & 1 & 1 & 13458 & 589 & 604 & cancer Dulmonar & C0242379 & $2014-10-25$ \\
7945 & 5 & 1 & 2 & 13459 & 3413 & 3432 & carcinoma de Dulmon & C0684249 & $2018-11-01$ \\
3000 & 0 & 0 & 4 & 13460 & 259 & 277 & carcinoma Dulmonar & C0684249 & $2016-03-18$ \\
18982 & 1 & 2 & 7 & 13461 & 752 & 776 & adenocarcinoma de Dulmon & C0152013 & $2011-05-30$ \\
4501 & 0 & 5 & 1 & 13462 & 617 & 645 & escamoso carcinoma de Dulmon & C0149782 & $2017-11-25$
\end{tabular}

Figure 5.1: Snapshot of the structured diagnosis events for a patient in the

Document database

\begin{tabular}{|lllllllll}
\hline document & section & paragraph & sentence & id & begin & end & stage & date \\
\hline 2376 & 3 & 1 & 0 & 678 & 134 & 137 & III & $2015-02-07$ \\
11235 & 0 & 1 & 6 & 677 & 417 & 420 & III & $2015-01-06$ \\
18982 & 1 & 2 & 7 & 678 & 778 & 780 & II & $2011-05-30$ \\
307891 & 0 & 1 & 1 & 679 & 11 & 14 & III & $2014-11-01$ \\
4891 & 1 & 1 & 1 & 680 & 3871 & 3874 & III & $2015-01-19$ \\
4891 & 16 & 1 & 0 & 681 & 5902 & 5905 & III & $2016-07-23$ \\
140423 & 9 & 8 & 0 & 682 & 2763 & 2766 & III & $2014-08-27$ \\
191 & 17 & 1 & 1 & 683 & 4341 & 4344 & III & $2016-12-14$ \\
137610 & 15 & 1 & 0 & 684 & 5470 & 5472 & II & $2012-06-25$ \\
37690 & 9 & 2 & 1 & 685 & 2321 & 2323 & II & $2016-06-06$ \\
107 & 0 & 0 & 7 & 686 & 371 & 373 & II & $2014-09-11$ \\
48230 & 8 & 4 & 2 & 687 & 2619 & 2622 & III & $2017-05-15$
\end{tabular}

Figure 5.2: Snapshot of the structured stage grouping events for a patient in the Document database

\begin{tabular}{|lllllllll}
\hline document & section & paragraph & sentence & id & begin & end & thm & date \\
\hline 137610 & 15 & 1 & 0 & 3672 & 5474 & 5482 & T2aN1M0 & $2012-06-25$ \\
171 & 2 & 4 & 0 & 3673 & 969 & 976 & DT2N1M0 & $2011-11-15$ \\
538 & 15 & 1 & 0 & 3674 & 1151 & 1161 & cT2acN1cM0 & $2013-01-29$ \\
617 & 5 & 3 & 0 & 3675 & 2297 & 2303 & T2N1M0 & $2011-05-30$
\end{tabular}

Figure 5.3: Snapshot of the structured TNM events for a patient in the Document database

all the duplicated information in order to find the exact occurrence date of events and also their evolution along the time.

In addition, although the recognition of medical events is one of the fundamental tasks of NLP, the judgment of medical events cannot be understood solely at sentence level. This is another challenge that needed to be tackled 
Chapter 5. Implementation and Deployment

5.2. Identifying the Temporal Order of Medical Events Across EHRs

\begin{tabular}{|c|c|c|c|c|c|c|c|c|c|c|}
\hline id & patient & birthDate & gender & category & subcategory & date & hospital & begin & end & text \\
\hline 7179 & 10335 & $1951-09-12$ & Female & Note & Anamnesis Cex & $2009-02-06$ & HUPHM & 0 & 2280 & SERVICIO DE ONCOLOGIA RADIOTERAPICA P... \\
\hline 15268 & 10335 & $1951-09-12$ & Female & Note & Evolución Méd Cex & $2009-02-06$ & HUPHM & 0 & 1878 & Paciente de 57 años. vista Dor orimera vez en ... \\
\hline 15266 & 10335 & $1951-09-12$ & Female & Note & Evolución Méd Cex & $2009-03-13$ & HUPHM & 0 & 216 & REVISION Sin sintomas. El dolor lumbar es inter... \\
\hline 15267 & 10335 & $1951-09-12$ & Female & Note & Evolución Méd Cex & $2009-04-28$ & HUPHM & 0 & 1182 & Informe de la comoaracion de las mamoarafias $r \ldots$ \\
\hline 22889 & 10335 & $1951-09-12$ & Female & Note & Nota de Uraencias & $2009-08-24$ & HUPHM & 0 & 732 & Traumatismo directo anteoie derecho v reaion a... \\
\hline 7180 & 10335 & $1951-09-12$ & Female & Note & Evolución Méd Cex & $2009-09-18$ & HUPHM & 0 & 416 & REVISION Refiere dolores en Dinchazo en mama... \\
\hline 30727 & 10335 & $1951-09-12$ & Female & Note & Evolución Méd Cex & $2009-11-11$ & HUPHM & 0 & 1087 & REVISIÓN Ecoarafia de mama: Paciente con cir... \\
\hline 11238 & 10335 & $1951-09-12$ & Female & Note & Evolución Méd Cex & $2009-12-04$ & HUPHM & 0 & 1963 & REVISIÓN MAMOGRAFIA BILATERAL: La Dacie... \\
\hline 30753 & 10335 & $1951-09-12$ & Female & Note & Nota de Rehabilitación & $2010-03-22$ & HUPHM & 0 & 569 & Dan stvle="font-size: laraer" $>$ Enviada oor M... \\
\hline 22865 & 10335 & $1951-09-12$ & Female & Note & St Cex & $2010-04-16$ & HUPHM & 0 & 256 & REVISIÓN Reexoloro lesión en reción femoral iz... \\
\hline 10 & 10335 & $1951-09-12$ & Female & Reoort & Informe de Seauimiento Consulta Externa & $2010-05-10$ & HUPHM & 0 & 1696 & SERVICIO: Oncolooia Radioteraoica FECHA CR... \\
\hline 11239 & 10335 & $1951-09-12$ & Female & Note & Evolución Méd Cex & $2010-05-10$ & HUPHM & 0 & 231 & Acude remitida desde Rehabilitación Dara oreau... \\
\hline 30728 & 10335 & $1951-09-12$ & Female & Note & Evolución Méd Cex & $2010-09-03$ & HUPHM & 0 & 444 & REVISIÓN Ha tenido cistitis v vresenta dolores ... \\
\hline 7181 & 10335 & $1951-09-12$ & Female & Note & Evolución Méd Cex & $2010-09-24$ & HUPHM & 0 & 1323 & VALORACIÓN ESTUDIOS No nuevos síntomas d... \\
\hline 19 & 10335 & 1951-09-12 & Female & Reoort & ta de Urae & $2011-05-30$ & HUPHM & 0 & 2596 & SERVICIO: Uraencias Generales FECHA INGRE... \\
\hline 18982 & 10335 & $1951-09-12$ & Female & Note & Evolución Médica Uraencias & $2011-05-30$ & HUPHM & 0 & 532 & Pruebas Comolementarias: Rx de ambos hombr... \\
\hline 26807 & 10335 & $1951-09-12$ & Female & Note & nesis Cex & $2011-05-30$ & HUPHM & 0 & 1696 & MC: artarlaai en lado izouierdo. AP: Refiere al... \\
\hline 11240 & 10335 & $1951-09-12$ & Female & Note & Evolución Méd Cex & $2011-07-29$ & HUPHM & 0 & 234 & REVISIÓN Dolor cocciaeo, oue va tenía oreviam... \\
\hline 18983 & 10335 & $1951-09-12$ & Female & Note & Evolución Méd Cex & $2011-08-04$ & HUPHM & 0 & 2087 & 30/05/2011 18:22:16 Anamnesis Cex Jessica P... \\
\hline 26808 & 10335 & $1951-09-12$ & Female & Note & Ev & $2011-08-10$ & HUPHM & 0 & 2526 & 30/05/2011 18:22:16 Anamnesis Cex Jessica P.. \\
\hline 11271 & 10335 & $1951-09-12$ & Female & Note & & -25 & IM & 0 & 389 & Paciente remitida oor dolor en cintura escaoular \\
\hline 26809 & 10335 & $1951-09-12$ & Female & Note & on Med Cex & $2011-08-25$ & HUPHM & 0 & 95 & Se recibe RM: Ninoun hallazoo Datolóaico. Remi... \\
\hline 15293 & 10335 & $1951-09-12$ & Female & Note & Comentario Enf Cex & $2011-08-25$ & HUPHM & 0 & 28 & Saco analítica oreoperatoria \\
\hline 34618 & 10335 & 1951-09-12 & Female & Note & ta de Uraencias & $2011-0$ & IUPHM & 0 & 1296 & strico Ap: Re \\
\hline
\end{tabular}

Figure 5.4: Snapshot of the structured information about the patients and their EHRs in the Document database

at this stage.

As the last point, since the the document database only provides insights to the information at document level (Figure 5.5) and does not facilitate the integration of information for patients thus, we cannot query the patient's medical time-line. Therefore, the integration of structured information is still required for reconstructing the patient's natural history.

\subsubsection{Design of the Solution}

To automatically find the exact date of lung cancer diagnosis and the evolution of tumor stage events from the patient's EHRs, two separate processes have been followed by the Timeline Constructor. In what follows, firstly, we describe the process designed to automatically detect the patient's diagnosis date and then, we explain how the evolution of tumor stage events is discovered.

Figure 5.6 presents the flowchart designed to identify the exact date of diagnosis event. As one can see, first of all, the Time-line Constructor reads the diagnosis events as input from Document database. Since, these events are mentioned in different sections of clinical reports (e.g., Diagnosis, Family Antecedents, Personal Antecedents, etc.) or various types of clinical notes (e.g., Nursery Comment, Microbiology Note, Emergency Note, etc.), it is important to identify which of these report sections and note types are most 
Chapter 5. Implementation and Deployment

5.2. Identifying the Temporal Order of Medical Events Across EHRs

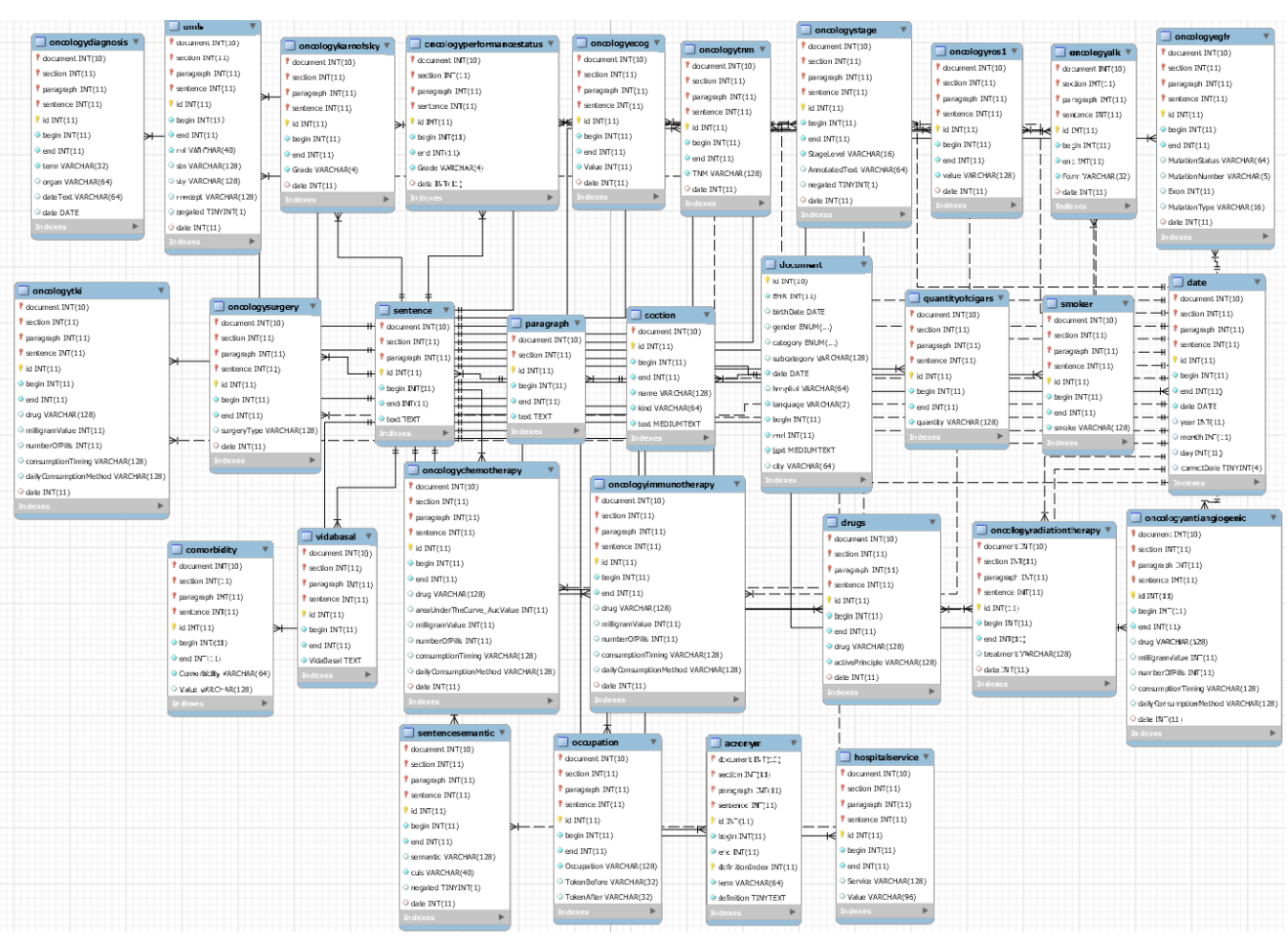

Figure 5.5: Snapshot of Document database design containing annotations

probable to contain non-redundant information. For this purpose, a list of pre-defined report sections and a list of note types were provided by clinicians of HUPHM for our implementation. Using this list, the Timeline Constructor filters diagnosis events. Then, it orders them in the ascending order, selects the earliest instance and discards the rest.

Figure 5.7 illustrates the process designed to automatically discover the temporal order of tumor stage events in order to generate their evolution on the medical timeline. As stage grouping and TNM codes are the two main standard cancer staging concepts, which are annotated and structured in the Document database, firstly, it is essential to map the values of one type to the another one. For this purpose, the Timeline Constructor by reading the TNM events from the database, it converts them to the stage grouping codes and stores the mapped values in the stage grouping table. 
Chapter 5. Implementation and Deployment

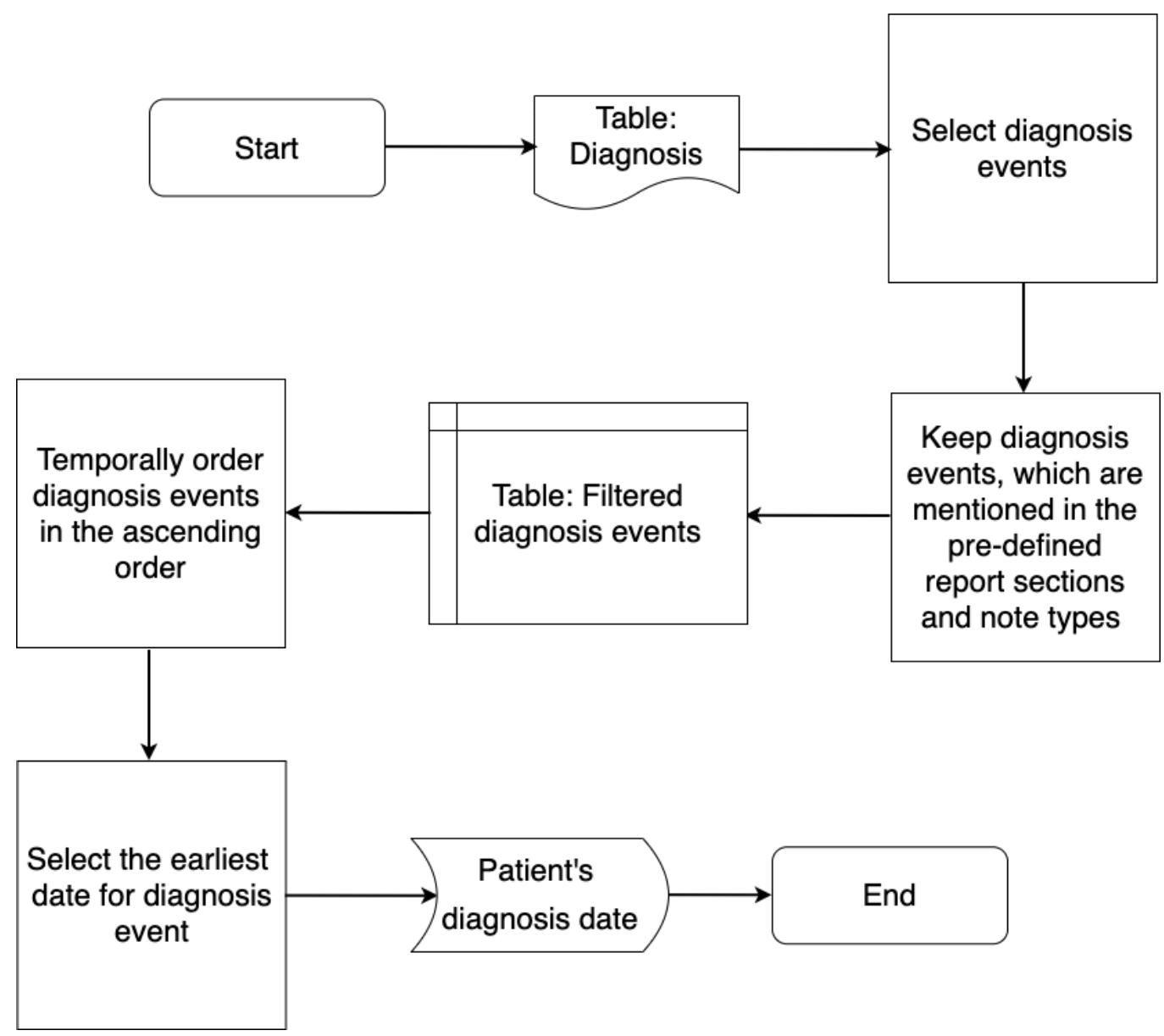

Figure 5.6: Flow chart for identifying the patient's diagnosis date

Afterwards, the Timeline Constructor accesses the stage grouping table to get the tumor stage codes as input. Then, it filters and keeps those, which are mentioned in the pre-defined sections of clinical reports and the types of clinical notes, provided in the list by HUPHM clinicians. Finally, it orders stage events in the ascending order, keeps the unique as well as the earliest instance of the same medical events and discard the rest of events. 
Chapter 5. Implementation and Deployment

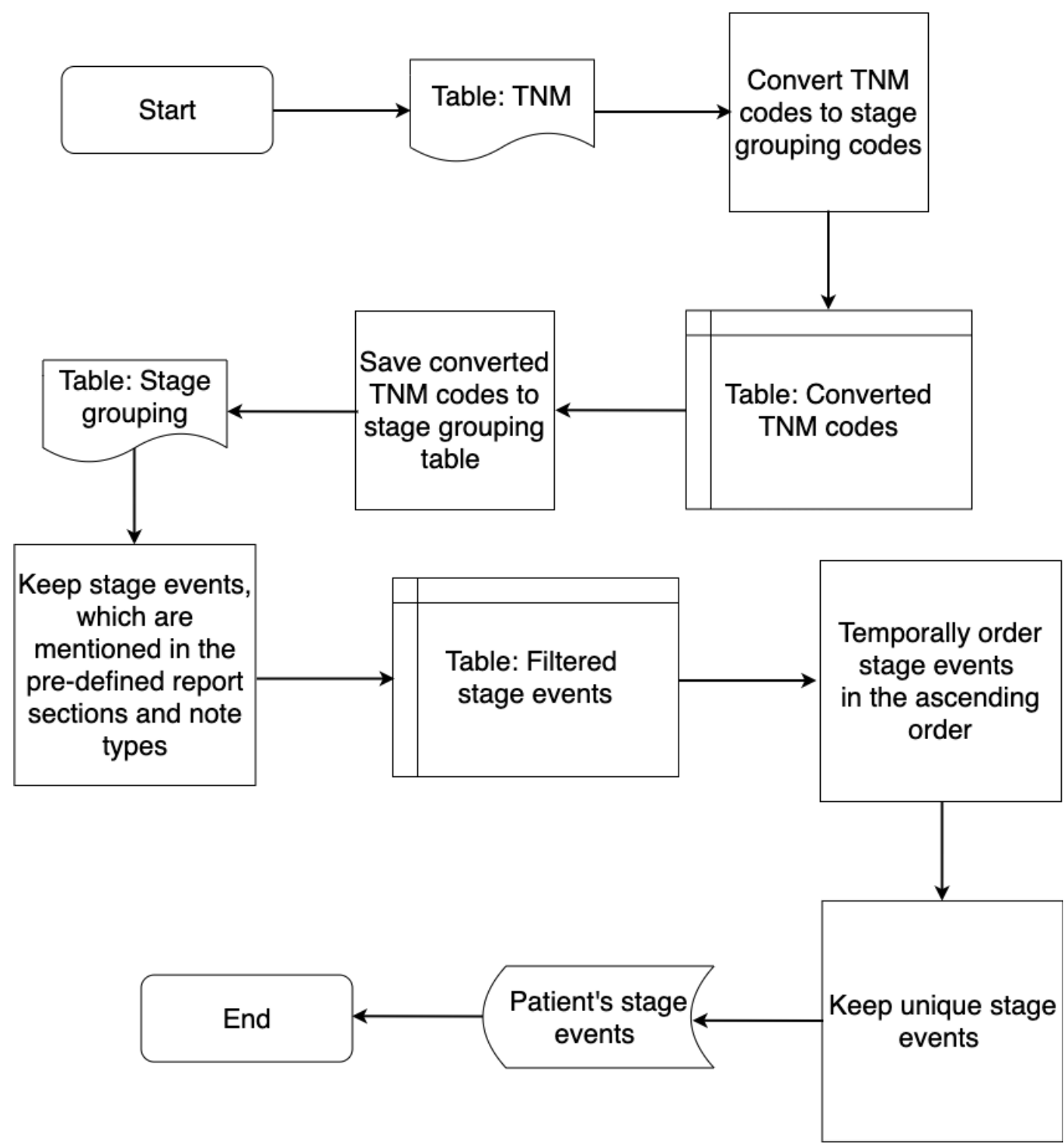

Figure 5.7: Flow chart for identifying the evolution of tumor stage events

\subsection{Deployment}

In this Section, we discuss the technologies used for deployment of our NLP framework. As one can see in Figure 5.8, the input to our framework is EHRs, stored in plain text files and the output is structured information 
Chapter 5. Implementation and Deployment

5.3. Deployment

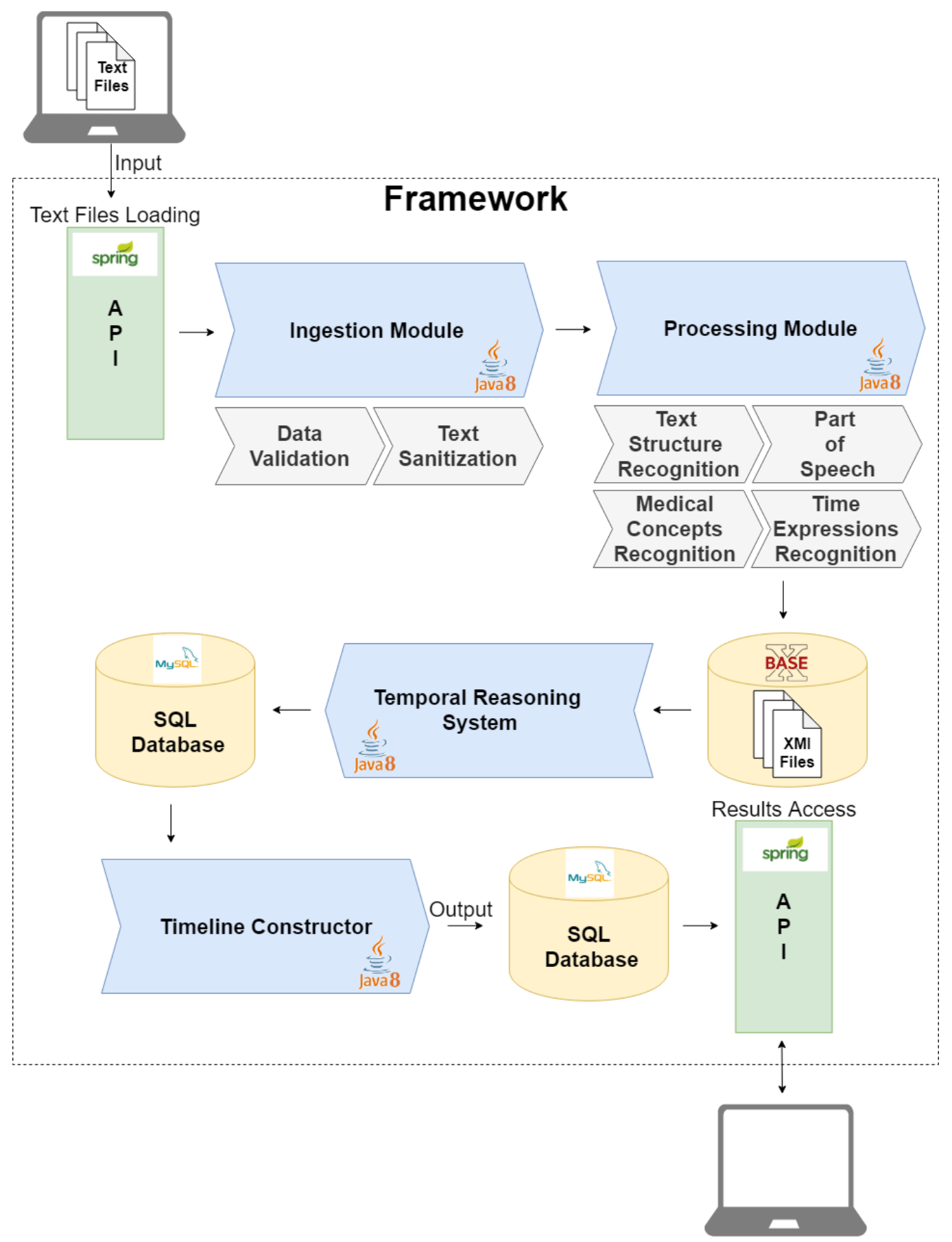

Figure 5.8: Technologies used to deploy our Framework 
Chapter 5. Implementation and Deployment

5.3. Deployment

about the patient's medical timeline, which can be retrieved in JavaScript Object Notation (JSON) format. Our framework is mainly composed of the following components:

1. APIs: two RESTful web services are developed over Java Spring [260] to allow other systems, platforms, dashboards, and web pages to connect to our NLP framework. Using the HTTP Post request to the first API, the plain text files can be provided as input to the framework for processing (example is provided in Listing 5.1). On the other hand, using the HTTP GET request to the second API, the natural histories of patients can be received in JSON file format (example is provided in Listing 5.2.

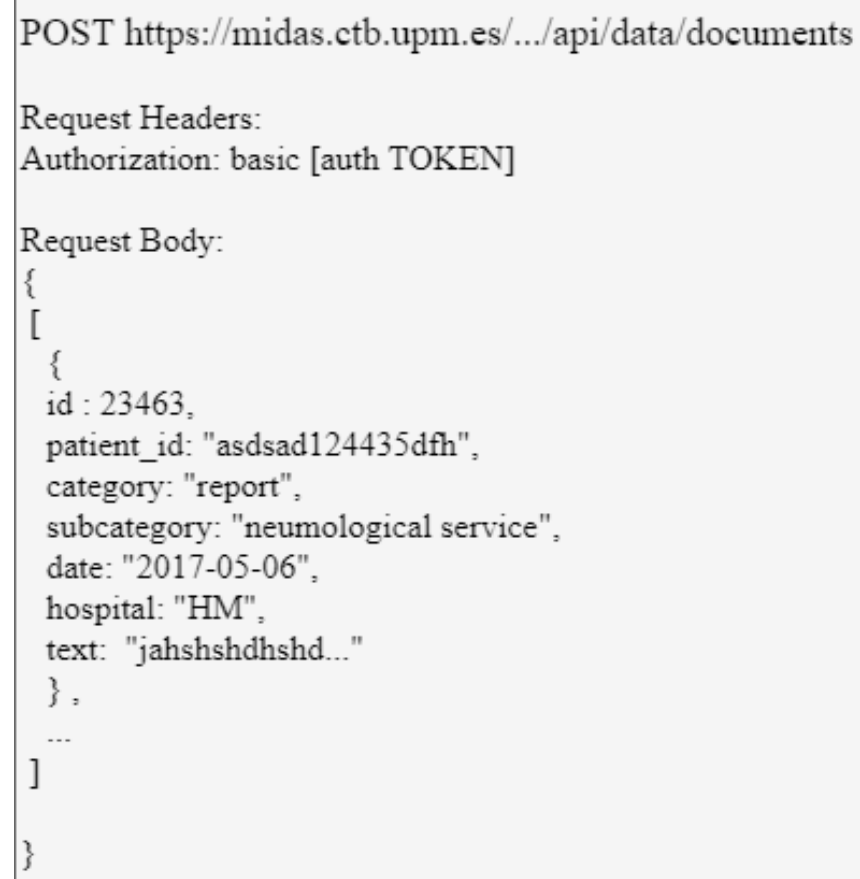

Listing 5.1: Example of making a HTTP Post request to our NLP framework 
Chapter 5. Implementation and Deployment

5.3. Deployment

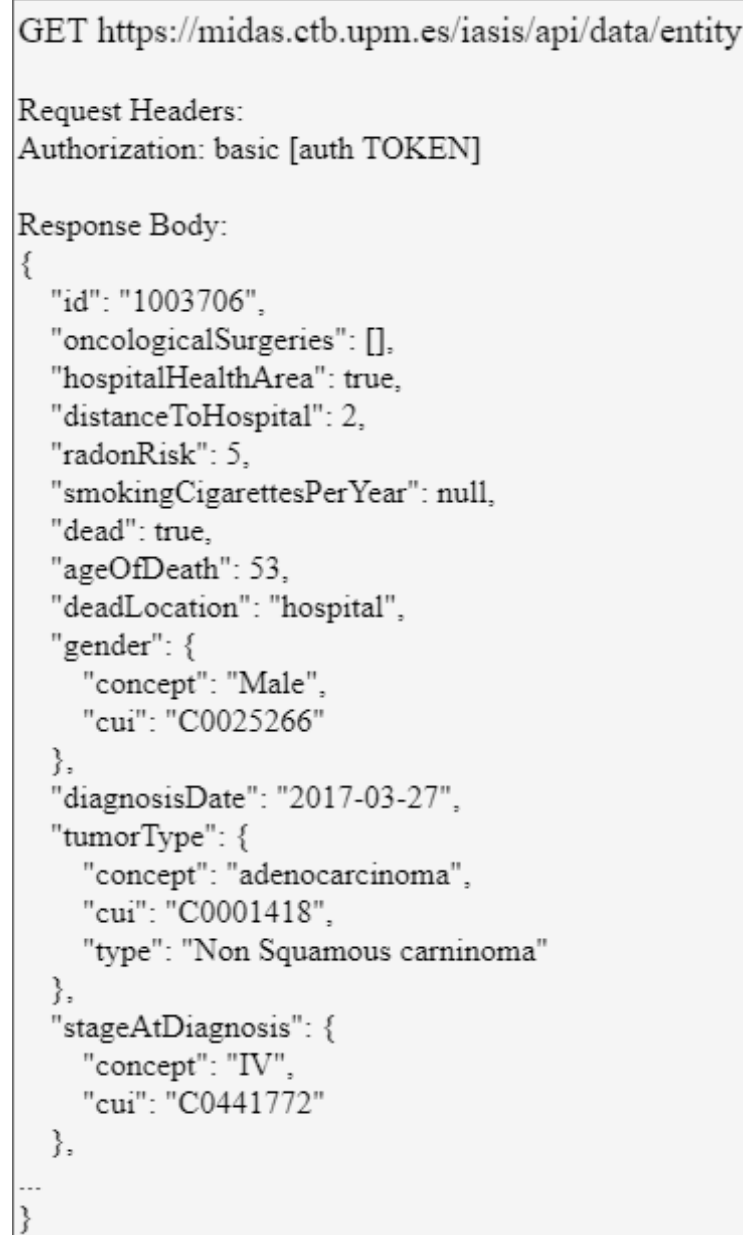

Listing 5.2: Example of making a HTTP GET request to our NLP framework

2. Ingestion Module: performs two main tasks:

- Data Validation: checks if every EHR is written in Spanish language and if it contains the information about the patient id, date of birth, gender and the document creation date. In addition, for each patient, it also checks for information consistency within EHRs. For example, it examines if the information about a patient's date of birth and gender is the same in all of the patient's documents. 
Chapter 5. Implementation and Deployment

5.3. Deployment

- Text Sanitization: is responsible for normalizing and harmonizing the structure of EHRs by removing accents from Spanish letters and deleting redundant new lines from clinical texts. This process will later help the rule-based annotators to have a higher accuracy in their implementation.

3. Processing Module: accepts the normalized text files of Ingestion Module as input, performs a set of NLP processes on them and then, formats its output as a set of XMI files, which will be stored into a BASEX database. This module is composed of a set of syntactic and semantic processing components, which are built over UIMA framework. These components are:

- Text Structure Recognition: splits a given input character sequence into the different tokens. These tokens constitute the minimum pieces of information in a sentence and they are usually words, punctuations, symbols, numbers, etc. Then, it statistically determines if a punctuation character marks the end of a sentence in order to detect the sentence boundaries. Finally, it recognizes paragraphs boundaries and sections in the free texts.

- Part of Speech: determines the corresponding syntactic word type of tokens based on the token itself and the context in which the token is.

- Medical Concepts Recognition: detects the NEs of medical concepts from clinical texts. Example of such concepts are disease diagnosis and tumor stage concepts.

- Time Expressions Recognition: identifies and normalizes time expressions mentioned in free texts of EHRs.

4. Temporal Reasoning System: reads EHRs from BASEX database and performs the identification of temporal relations mentioned between medical concepts and time expressions in clinical texts. The output obtained from this stage is stored into a MySQL structured relational database, which is organized mimicking the internal structure of documents.

5. Timeline Constructor: processes the structured information of MySQL database for yielding the patient's natural history on a medical time-line. 
Chapter 5. Implementation and Deployment 5.4. Application

The results of this module are also stored into a MySQL structured relational database.

we have chosen Java8 as the programming language for the development of our NLP framework. In addition, to prepare the production version of this framework, each of the aforementioned components and databases are implemented in the individual Docker containers [261] - see Figure 5.9.

\subsection{Application}

Our NLP framework has several applications in medical informatics such as improving QA process, supporting clinical decision making, calculating the patient's survival time, to name a few. In this section, we introduce the application of our framework for QA process and patient's survival time calculation.

We discuss a real world example of utilizing our NLP framework in a web platform, named iASiS. The iASiS platform uses the output generated by our framework for further addressing the clinicians needs in performing statistical analysis and QA process on the natural histories of lung cancer patients. This platform includes two main components for performing the aforementioned tasks: (1) Visual Analytics Tool, which presents the knowledge related to the populations of patients; and (2) Question Answering Tool, which replies to the user questions about the data.

In what follows, we will present the snapshots of iASiS platform. Figure 5.10 presents the login dashboard. To enter to the platform, the end user needs to enter his credentials in the Email and password fields.

Once, the end user logged in to the system, he can access the knowledge related to the population of patients in analytics dashboard - see Figure 5.11. For this purpose, the user should select a specific population through the selection filters provided in the left side of the page (e.g., gender, age at diagnosis, histology, stage of tumor, etc.). After the selection process is 


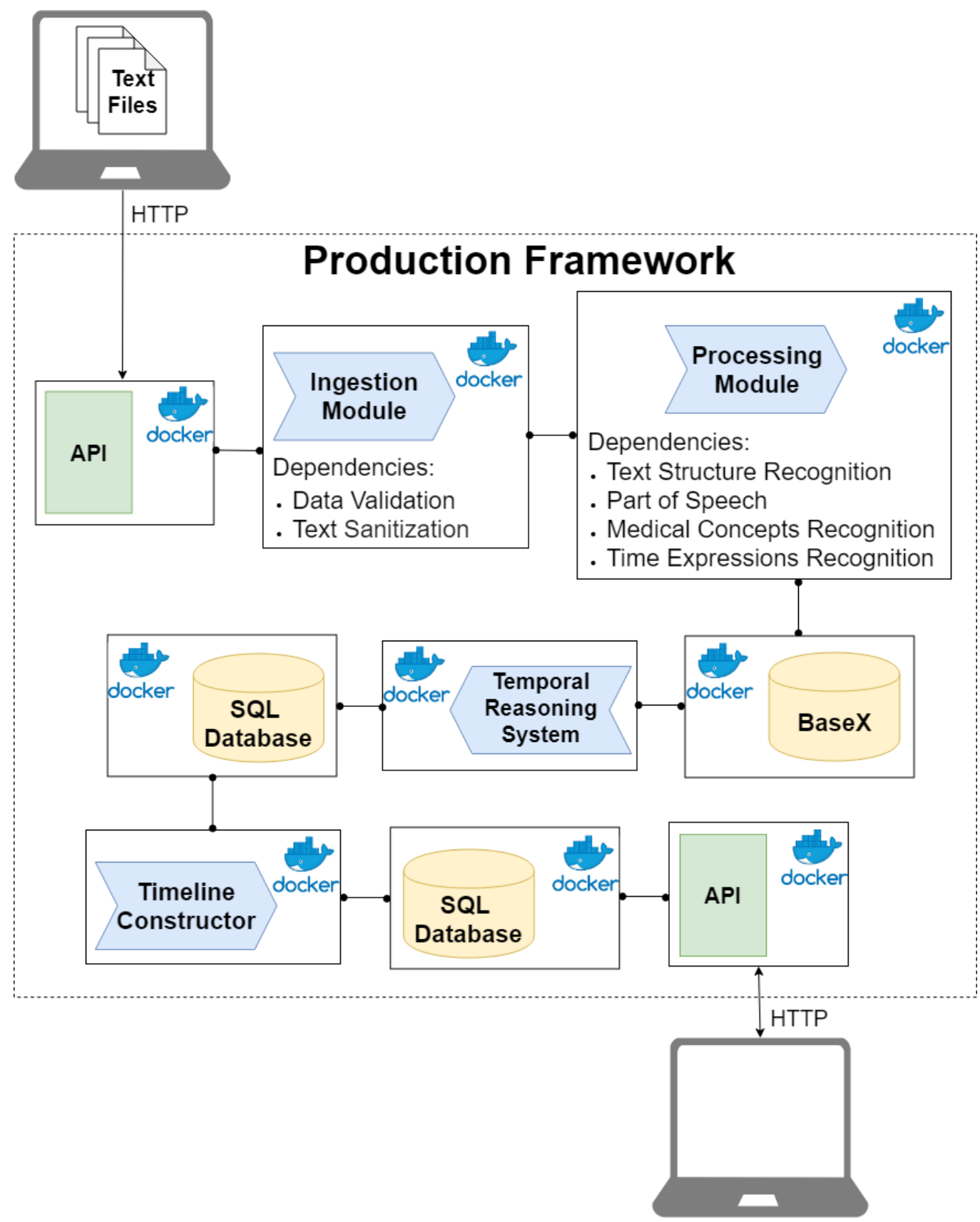

Figure 5.9: Overview of the production framework 
Chapter 5. Implementation and Deployment

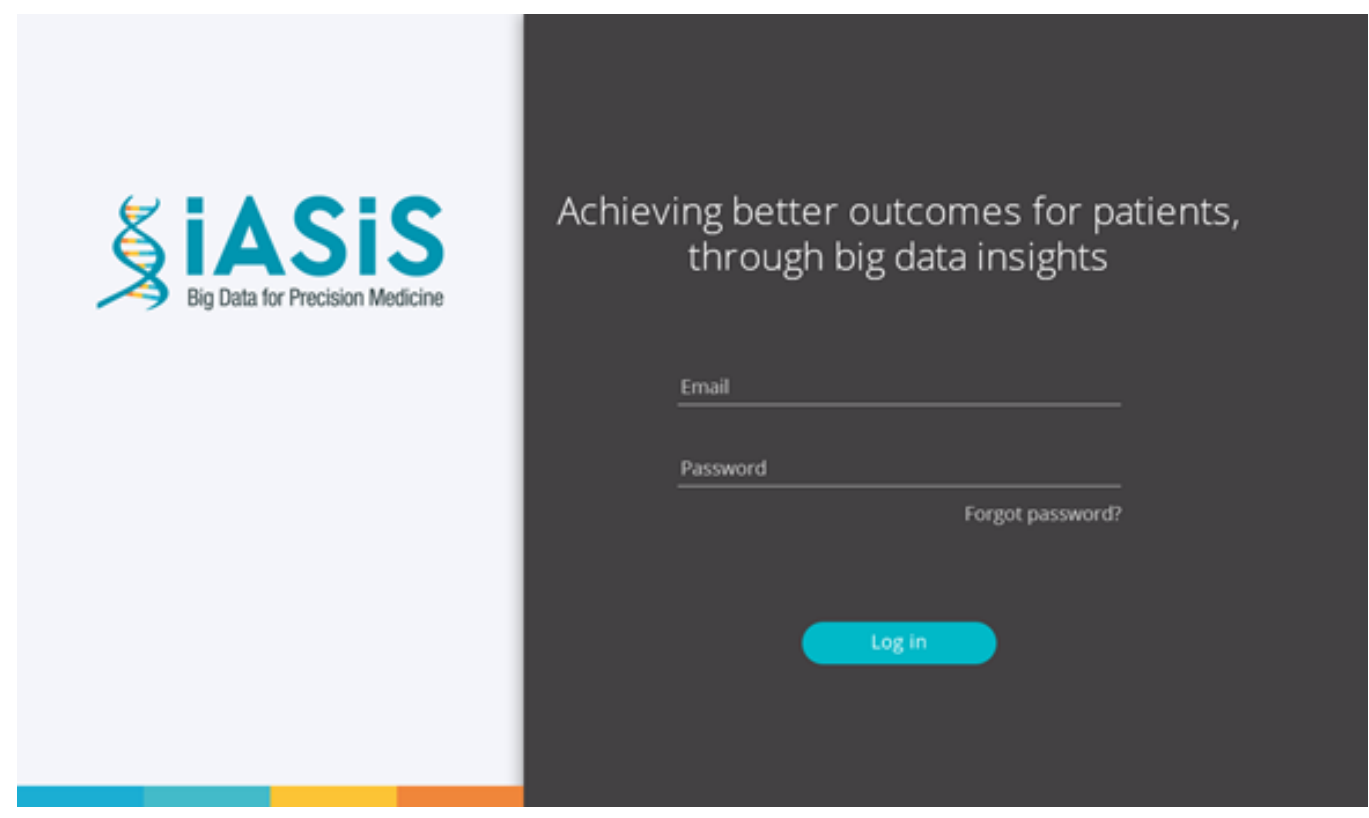

Figure 5.10: Login dashboard of iASiS platform

completed, the user can click on the "Apply filters" button in order to see the results.

Figure 5.12 presents an example in which the user have chosen specific selection filters on the right side of the page and the results obtained from this selection on the left side of the page. In addition, the user can also click on the "View justification" link for each parameter to access the bibliographical links and the survival probability image related to the justification of the confidence score (Figure 5.13). For example the survival curve generated for patients when the parameter stage of the tumor is selected by the user, is presented in the Figure 5.14 .

\subsection{Discussion}

Applications of Big data in healthcare deliveries facilitates a high potential to improve the overall efficiency and the quality of the care delivery. In this 
Chapter 5. Implementation and Deployment

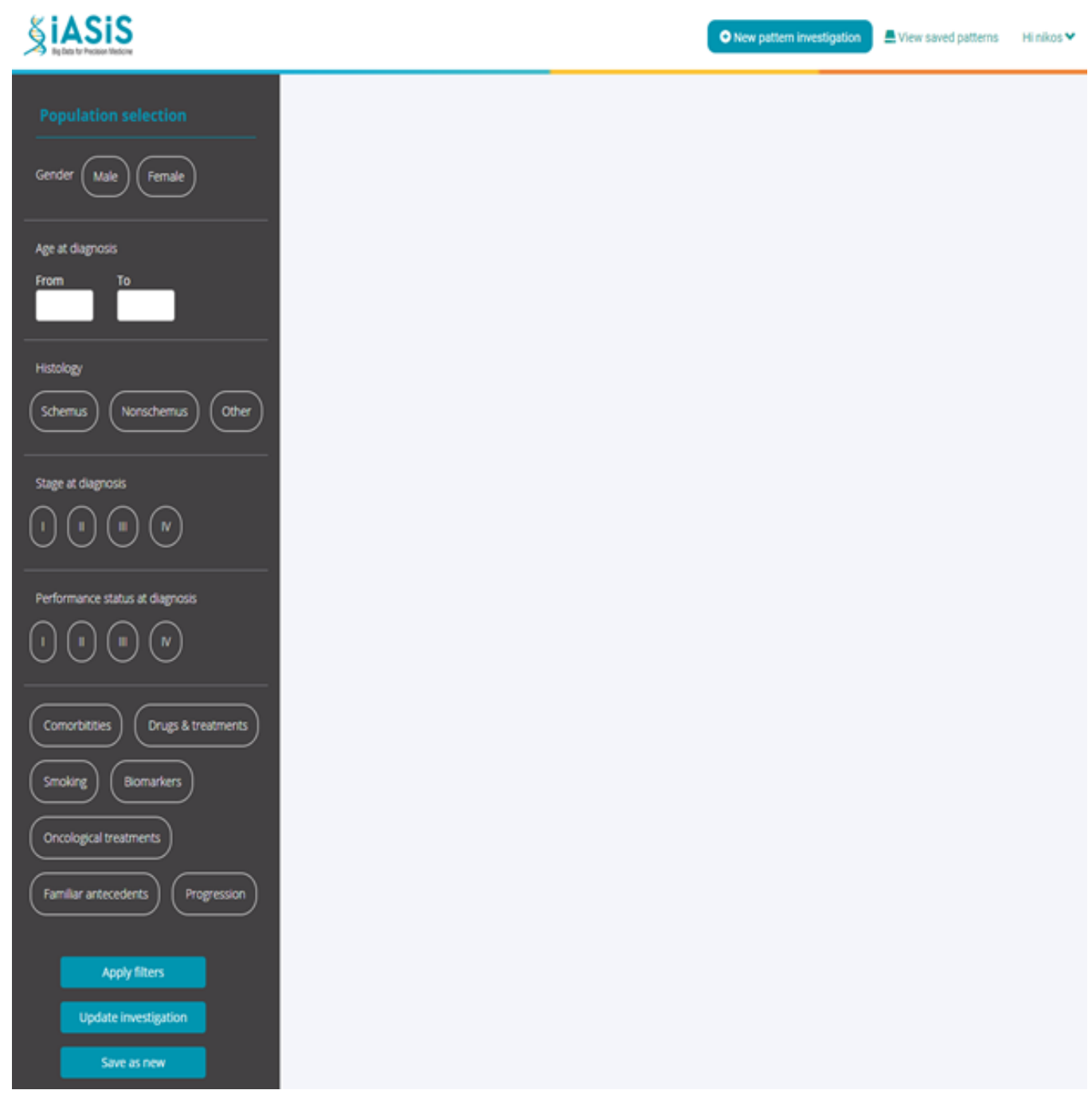

Figure 5.11: Analytics dashboard of iASiS platform 
Chapter 5. Implementation and Deployment

5.5. Discussion
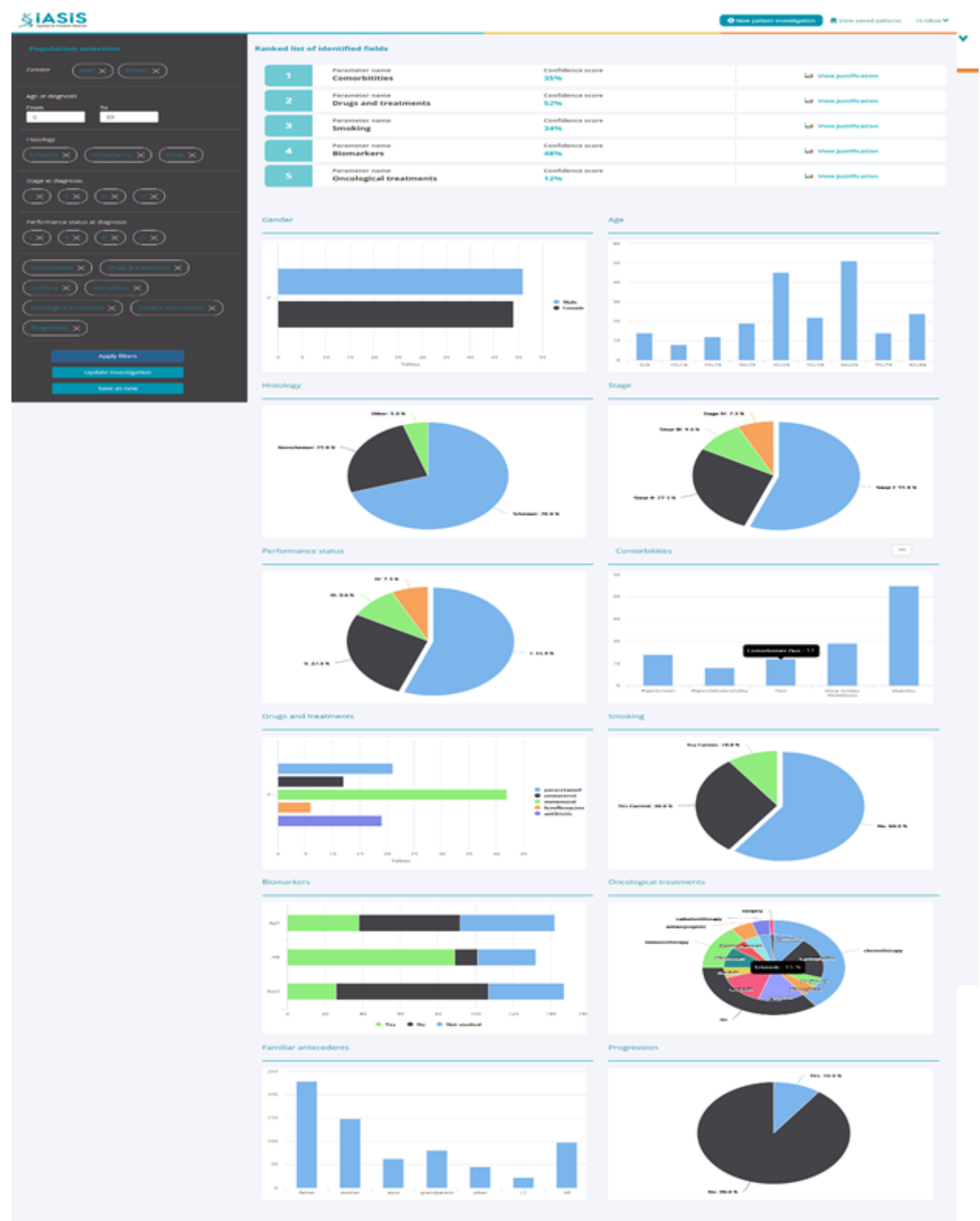

Figure 5.12: An Example of population selection and results generation in analytics dashboard 
Chapter 5. Implementation and Deployment

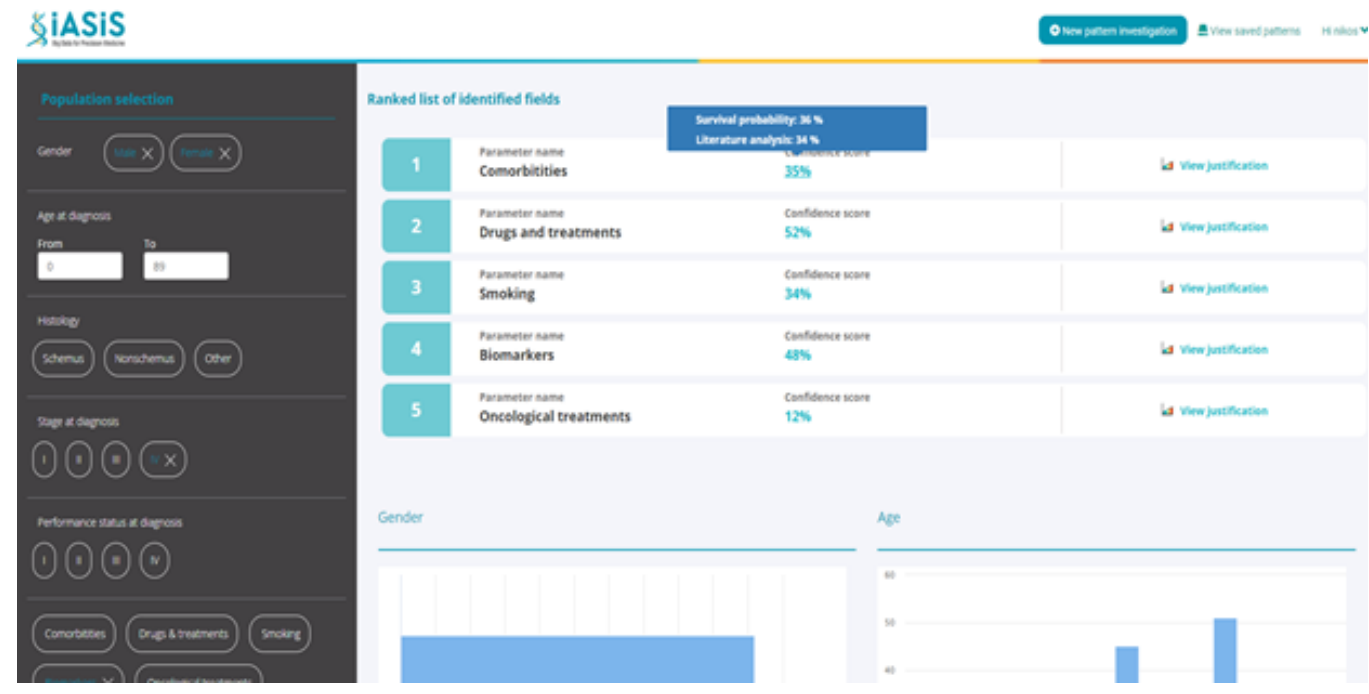

Figure 5.13: Selection of parameters to access bibliographical links and the survival probability image

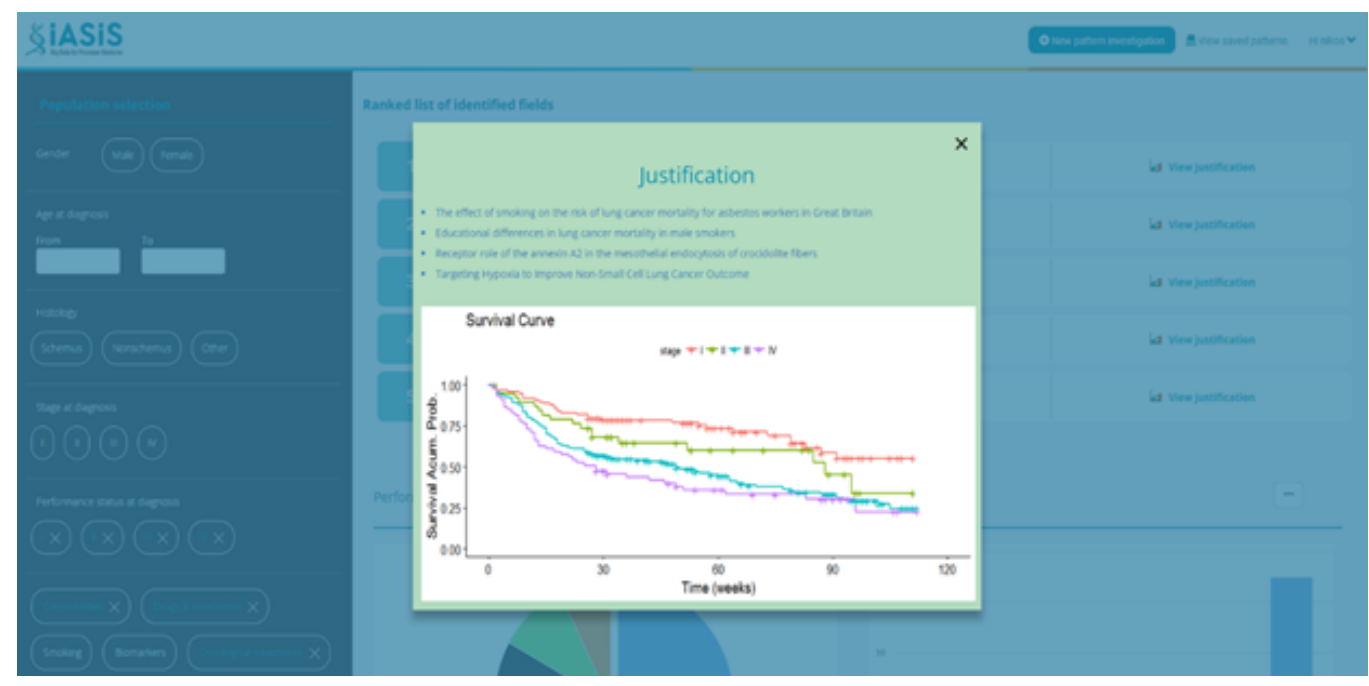

Figure 5.14: Survival probability of lung cancer patients based on the parameter of tumor stage 
Chapter 5. Implementation and Deployment

5.5. Discussion

Chapter, we have analyzed the problem of identifying the temporal order of structured medical events and discussed the implementation details to tackle it. We have also explained the technologies used for the deployment of our NLP framework and its application in the healthcare sector. It has been shown that despite of the huge work require to structure and order events on a medical timeline prior to analyses, this is a huge step towards personalized medicine. 


\section{Conclusions and Future Work}

With the increased availability of EHRs over the past decade, constructing the patient's natural history has gained a lot of attention by the medical informatics community. In the line of this research and in this Thesis, we presented the methods for extraction of specific medical concepts related to lung cancer domain, time expressions and temporal relations from EHRs written in Spanish.

\subsection{Conclusions}

This thesis introduced new methods to apply NLP techniques for analyzing EHRs. During the development of the Thesis, a framework has been designed and developed to reconstruct the natural history of patients, i.e. the evolution of medical events on a timeline from EHRs written in Spanish.

Starting from an initial set of raw textual data provided as Spanish EHRs, a set of processes is defined in the light of research questions reported in Section 1.2.

1. Can medical concepts written in natural language be automatically annotated? In the Thesis, to encode, structure and extract lung cancer concepts of tumor mutation status, tumor stage and performance status from clinical text, a set of rule-based annotators is designed, 
Chapter 6. Conclusions and Future Work

6.1. Conclusions

developed and tested. These annotators are built over Apache UIMA framework.

2. Can time expressions mentioned in free texts be transformed into a structured and normalized format? Events happen in time and thus, time is the main parameter to interpret events. In the context of the Thesis, a rule-based Temporal Tagger is developed using UIMA framework for extraction and normalization of time expressions mentioned in free texts. The Temporal Tagger is able to extract various time expressions, filter those that are not likely to be time-related, resolve them with respect to a reference date, and finally normalize them to a standard date format. This module is designed to be domain independent.

3. Can temporal relations between medical concepts and time expressions be identified from EHRs? In the Thesis, a Temporal Reasoning System is developed for identification of temporal relations at sentence, section and document level of EHRs. This system makes use of the dependency parsing technique and a set of rules for its implementation and can be used directly in other domains without any modifications or adaption.

Consequently, the hypothesis presented in the Thesis have been verified through implementation of the aforementioned processes and with the conduction of various experiments. These hypothesis were:

$\checkmark$ Hypothesis 1: The NLP rule-based techniques can be used to detect medical concepts written in natural language.

$\checkmark$ Hypothesis 2: The NLP rule-based techniques can be used to recognize and normalize time expressions written in natural language..

$\checkmark$ Hypothesis 3: The Dependency parsing technique combined with an NLP rule-based approach can be used for linking medical concepts to time expressions in clinical documents. 
Chapter 6. Conclusions and Future Work 6.2. Future Lines

\subsection{Future Lines}

While several challenges have been successfully tackled in this work, some new challenges have also emerged, which we hope will be the target of the research beyond the $\mathrm{PhD}$. Among them, some of the most important ones are:

1. Although the development of approaches to detect specific medical concept related to lung cancer domain from EHRs is successfully implemented in the Thesis, in clinical NLP, the meaning of the entities can be heavily affected by the modifiers like negation. Creation of new approaches to detect negation from Spanish clinical texts could lead to an improvement in the detection of medical events.

2. Our proposed solution for annotation of time expressions has outperformed the recent studies in analysis of Spanish texts. However, there are still rooms for improvements by creating new approaches to annotate duration, interval, set and combined time expressions.

3. Our Temporal Reasoning System provides the instant-based representation of medical events. However, for some medical events such as treatment events, it is important to know their time-interval, i.e. their starting and ending time points. This research could be performed as an improvement to temporal relation discovery.

4. There are different types of discourse relations between textual units. These include comparison, expansion, contingency, casual and temporal. We have focused on the detection of temporal relations between medical events and time expressions mentioned in Spanish EHRs. However, identifying different types of discourse relations to represent more complex non-linear relationships with medical events on the generated medical timeline could improve the timeline.

5. The output of our framework for reconstructing the natural histories of lung cancer patients is stored into a relational database. However, designing a cognitively engineered display for visualizing the timeline in an intuitive manner will be of immense use to the clinical community. 
Chapter 6. Conclusions and Future Work 6.2. Future Lines

6. The improvement of the framework by supporting multilingualism. This is a significant step toward integrating the framework in different countries by tackling the problem of understanding various languages. 


\section{Bibliography}

[1] "The Digitization of the World From Edge to Core Mankind is on a quest to digitize the world." https: //www.seagate.com/files/www-content/our-story/trends/ files/idc-seagate-dataage-whitepaper.pdf. [Accessed: 20-Mar2019].

[2] E. Menasalvas, A. Rodriguez-Gonzalez, R. Costumero, H. Ambit, and C. Gonzalo, "Clinical Narrative Analytics Challenges," in International Joint Conference on Rough Sets, vol. 9920, pp. 23-32, 2016.

[3] "Apache UIMA." https://uima.apache.org/. [Accessed: 26-Feb2019].

[4] "UIMA Tutorial and Developers' Guides." https://uima.apache. org/d/uimaj-2.10.2/tutorials\{_\}and\{_\}users\{_\}guides.html. [Accessed: 26-Feb-2019].

[5] F. C. Detterbeck, "The eighth edition TNM stage classification for lung cancer: What does it mean on main street?," Journal of Thoracic and Cardiovascular Surgery, vol. 155, no. 1, pp. 356-359, 2018.

[6] "UDPipe." http://lindat.mff.cuni.cz/services/udpipe/. [Accessed: 20-Mar-2019].

[7] "Digital Data Storage is Undergoing Mind-Boggling Growth." https: //www . eetimes. com/author . asp? section $\left\{_{-}\right\}$id $=36\{\&\}$ doc $\left\{\left\{_{-}\right\}\right.$id= 1330462. [Accessed: 20-Mar-2019].

[8] "How EHR adoption is hindering healthcare big data, analytics." https://ehrintelligence.com/news/ how-ehr-adoption-is-hindering-healthcare-big-data-analytics. [Accessed: 21-Mar-2019]. 
[9] M. I. Xierali., C.-J. Hsiao, J. C. Puffer, L. A. Green, J. C. B. Rinaldo, A. W. Bazemore, M. T. Burke, and R. L. Phillips, "The Rise of Electronic Health Record Adoption Among Family Physicians," Ann Fam Med, vol. 11, no. 1, pp. 14-19, 2013.

[10] O. Ben-Assuli, "Electronic health records, adoption, quality of care, legal and privacy issues and their implementation in emergency departments," Health Policy, vol. 119, no. 3, pp. 287-297, 2015.

[11] "Office-based Physician Electronic Health Record Adoption." https://dashboard.healthit.gov/quickstats/pages/ physician-ehr-adoption-trends.php. [Accessed: 20-Mar-2019].

[12] "The Digital Doctor is "In" Accenture Eight-Country Survey of Doctors Shows Significant Increase in Healthcare IT Usage." https:// www .accenture.com/t20161118T020227Z__w__/us-en/_acnmedia/ Accenture/Conversion-Assets/DotCom/Documents/Global/PDF/ Industries_11/Accenture-Digital-Doctor-Is-In.pdf. [Accessed: 20-Mar-2019].

[13] "Big Data in Healthcare - the Challenges and the Promise - NEJM Catalyst." https://catalyst.nejm.org/big-data-healthcare/. [Accessed: 21-Mar-2019].

[14] S. Velupillai, H. Suominen, M. Liakata, A. Roberts, A. D. Shah, K. Morley, D. Osborn, J. Hayes, R. Stewart, J. Downs, W. Chapman, and R. Dutta, "Using clinical Natural Language Processing for health outcomes research: Overview and actionable suggestions for future advances," Journal of Biomedical Informatics, vol. 88, pp. 11-19, 2018.

[15] D. Reichert, D. Kaufman, B. Bloxham, H. Chase, and N. Elhadad, "Cognitive analysis of the summarization of longitudinal patient records.," in AMIA ... Annual Symposium proceedings / AMIA Symposium. AMIA Symposium, vol. 2010, pp. 667-671, 2010.

[16] Z. Luo, S. B. Johnson, A. M. Lai, and C. Weng, "Extracting Temporal Constraints from Clinical Research Eligibility Criteria Using Conditional Random Fields," in AMIA ... Annual Symposium proceedings / AMIA Symposium, pp. 843-852, 2011. 
[17] D. Demner-fushman, W. W. Chapman, and C. J. Mcdonald, "What can Natural Language Processing do for Clinical Decision Support?," Journal of biomedical informatics, vol. 42, no. 5, pp. 760-772, 2010.

[18] V. ZADNIK, T. ŽAGAR1, and M. PRIMIC ŽAKELJ, "Cancer Patients' Survival: Standard Calculation Methods And Some Considerations Regarding Their Interpretation," Zdr Varst, vol. 55, no. 2, pp. 144-151, 2016.

[19] E. Pons, L. M. M. Braun, M. M. G. Hunink, and J. A. Kors, "Natural Language Processing in Radiology: A Systematic Review," Radiology, vol. 279, no. 2, pp. 329-343, 2016.

[20] J. Manuel Bonet, R. Rodríguez-Ponga Salamanca, J. María Martínez Alonso, R. Bueno Hudson, M. López-Vega, and D. Fernández Vítores, "EL ESPAÑOL: UNA LENGUA VIVA," tech. rep., Instituto Cervantes, 2019.

[21] R. C. Moreno, C. G. Martín, and E. M. Ruiz, "TIDA: A spanish EHR semantic search engine," in 8th International Conference on Practical Applications of Computational Biology $\& 3$ Bioinformatics (PACBB 2014), pp. 235-242, 2014.

[22] E. M. Ruiz, J. M. Tuñas, G. Bermejo, C. G. Martín, A. Rodríguezgonzález, M. Zanin, C. G. D. Pedro, M. Méndez, O. Zaretskaia, J. Rey, C. Parejo, J. Luis, C. Bermudez, and M. Provencio, "Profiling Lung Cancer Patients Using Electronic Health Records," Journal of Medical Systems, vol. 42, no. 7, p. 126, 2018.

[23] I. Hernandez Medrano, J. Tello Guijarro, C. Belda, A. Urena, I. Salcedo, L. Espinosa-Anke, and H. Saggion, "Savana: Re-using Electronic Health Records with Artificial Intelligence," International Journal of Interactive Multimedia and Artificial Intelligence, vol. 4, no. 7, p. 8, 2018.

[24] D. Ferrucci and A. Lally, "UIMA: An architectural approach to unstructured information processing in the corporate research environment," Natural Language Engineering, vol. 10, no. 3-4, pp. 327-348, 2004.

[25] A. X. Chang and C. D. Manning, "SUTime: A library for recognizing and normalizing time expressions.," in Proceedings of the 8th Inter- 
national Conference on Language Resources and Evaluation, no. iii, pp. 3735-3740, 2012.

[26] "The Stanford Natural Language Processing Group." https://nlp. stanford.edu/software/sutime.html. [Accessed: 27-Feb-2019].

[27] J. Strötgen, J. Zell, and M. Gertz, "Heideltime: Tuning english and developing spanish resources for tempeval-3," in Second Joint Conference on Lexical and Computational Semantics ( $\left.{ }^{*} S E M\right)$, Volume 2: Proceedings of the Seventh International Workshop on Semantic Evaluation (SemEval 2013), vol. 2, pp. 15-19, 2013.

[28] "Database Research Group: HeidelTime Demonstration." https: //heideltime.ifi.uni-heidelberg.de/heideltime/. [Accessed: 27Feb-2019].

[29] M. Straka, "UDPipe: Trainable Pipeline for Processing CoNLL-U Files Performing Tokenization, Morphological Analysis, POS Tagging and Parsing," in Proceedings of the Tenth International Conference on Language Resources and Evaluation (LREC'16), pp. 4290-4297, 2016.

[30] M. Straka and J. Straková, "Tokenizing, POS Tagging, Lemmatizing and Parsing UD 2.0 with UDPipe," in Proceedings of the CoNLL 2017 Shared Task: Multilingual Parsing from Raw Text to Universal Dependencies, vol. 2, pp. 88-99, 2017.

[31] H. Llorens, E. Saquete, and B. Navarro, "TIPSem (English and Spanish): Evaluating CRFs and semantic roles in TempEval-2," in SemEval '10 Proceedings of the 5th International Workshop on Semantic, no. July, pp. 284-291, 2010.

[32] "iASiS." https://project-iasis.eu/. [Accessed: Feb-01-2019].

[33] G. Moharasar and T. B. Ho, "A semi-supervised approach for temporal information extraction from clinical text," in 2016 IEEE RIVF International Conference on Computing and Communication Technologies: Research, Innovation, and Vision for the Future, RIVF 2016 Proceedings, pp. 7-12, 2016.

[34] "Cancer." https://www.who.int/news-room/fact-sheets/detail/ cancer. [Accessed: 26-Feb-2019]. 
[35] " 1 in 4 deaths caused by cancer in the EU28 - Product - Eurostat." https://ec.europa.eu/eurostat/web/products-press-releases/ -/3-25112014-BP. [Accessed: 26-Feb-2019].

[36] R. Luengo-fernandez, J. Leal, A. Gray, and R. Sullivan, "Economic burden of cancer across the European Union : a population-based cost analysis," The Lancet Oncology, vol. 14, no. 12, pp. 1165-1174, 2013.

[37] D. Shlomi, N. Peled, Y. A. Schwarz, G. W. Soo Hoo, R. K. Batra, G. Fink, T. Kaplan, L. Cohen, S. Mollan, and W. R. Burfeind, "Noninvasive early detection of malignant pulmonary nodules by FISH-based sputum test," Cancer Genetics, vol. 226-227, pp. 1-10, 2018.

[38] A. Zaman and T. G. Bivona, "Emerging application of genomics-guided therapeutics in personalized lung cancer treatment.," Annals of translational medicine, vol. 6, no. 9, p. 160, 2018.

[39] "Molecular Profiling of Lung Cancer - My Cancer Genome." https: //mycancergenome.org/content/disease/lung-cancer/. [Accessed: 26-Feb-2019].

[40] "NCI Dictionary of Cancer Terms - National Cancer Institute." https: //www.cancer.gov/publications/dictionaries/cancer-terms. [Accessed: 26-Feb-2019].

[41] T. Ahmadzada, S. Kao, G. Reid, M. Boyer, A. Mahar, and W. Cooper, "An Update on Predictive Biomarkers for Treatment Selection in NonSmall Cell Lung Cancer," Journal of Clinical Medicine, vol. 7, no. 6, p. 153, 2018.

[42] M. G. Oser, M. J. Niederst, L. V. Sequist, and J. A. Engelman, "Transformation from non-small-cell lung cancer to small-cell lung cancer: Molecular drivers and cells of origin," The Lancet Oncology, vol. 16, no. 4, pp. e165-e172, 2015.

[43] T. Iwahara, J. Fujimoto, D. Wen, R. Cupples, N. Bucay, T. Arakawa, S. Mori, B. Ratzkin, and T. Yamamoto, "Molecular characterization of ALK, a receptor tyrosine kinase expressed specifically in the nervous system," Oncogene, vol. 14, no. 4, pp. 439-449, 1997. 
[44] V. M. Rimkunas, K. E. Crosby, D. Li, Y. Hu, M. E. Kelly, T. L. Gu, J. S. Mack, M. R. Silver, X. Zhou, and H. Haack, "Analysis of receptor tyrosine kinase ROS1-positive tumors in non-small cell lung cancer: Identification of a FIG-ROS1 fusion," Clinical Cancer Research, vol. 18, no. 16, pp. 4449-4457, 2012.

[45] "Implementation of AJCC 8th Edition Cancer Staging System." https://cancerstaging.org/About/news/Pages/ Implementation-of-AJCC-8th-Edition-Cancer-Staging-System . aspx. [Accessed: 26-Feb-2019].

[46] K. S. Mak, A. C. Van Bommel, C. Stowell, J. L. Abrahm, M. Baker, C. S. Baldotto, D. R. Baldwin, D. Borthwick, D. P. Carbone, A. B. Chen, J. Fox, T. Haswell, M. Koczywas, B. D. Kozower, R. J. Mehran, F. M. Schramel, S. Senan, R. G. Stirling, J. P. Van Meerbeeck, M. W. Wouters, and M. D. Peake, "Defining a standard set of patient-centred outcomes for lung cancer," European Respiratory Journal, vol. 48, no. 3, pp. 852-860, 2016.

[47] "Performance Scales: Karnofsky \& ECOG Scores Practice tools - OncologyPRO." http://oncologypro.esmo.org/ Oncology-in-Practice/Practice-Tools/Performance-Scales. [Accessed: 26-Feb-2019].

[48] Oken MM, Creech RH, Tormey DC, and E. al, "Toxicity and response criteria of the Eastern Cooperative Oncology Group," Am J Clin Oncol, vol. 5, no. 6, pp. 649-55, 1982.

[49] Y. Wang, L. Wang, M. Rastegar-Mojarad, S. Moon, F. Shen, N. Afzal, S. Liu, Y. Zeng, S. Mehrabi, S. Sohn, and H. Liu, "Clinical information extraction applications: A literature review," Journal of Biomedical Informatics, vol. 77, no. June 2017, pp. 34-49, 2018.

[50] "SNOMED - Home _ SNOMED International." https://www.snomed org/. [Accessed: 26-Feb-2019].

[51] B. L. Humphreys and D. A. B. Lindberg, "The UMLS@ project: Making the conceptual connection between users and the information they need," Bull Med Libr Assoc, vol. 81, no. April 1993, pp. 170-177, 1993. 
[52] "Unified Medical Language System (UMLS)." http://www.nlm.nih. gov/research/umls/. [Accessed: 26-Feb-2019].

[53] H. Cunningham, "GATE , a General Architecture for Text Engineering," Computers and the Humanities, vol. 36, pp. 223-254, 2002.

[54] "GATE." https://gate.ac.uk/. [Accessed: 26-Feb-2019].

[55] "protégé." https://protege.stanford.edu/products.php. [Accessed: 26-Feb-2019].

[56] K. Jensen, C. Soguero-Ruiz, K. Oyvind Mikalsen, R. O. Lindsetmo, I. Kouskoumvekaki, M. Girolami, S. Olav Skrovseth, and K. Magne Augestad, "Analysis of free text in electronic health records for identification of cancer patient trajectories," Scientific Reports, vol. 7, pp. 1-12, 2017.

[57] S. M. Meystre, G. K. Savava, K. C. Kipper-schuler, and J. F. Hurdle, "Extracting information from textual documents in the electronic health record: a review of recent research," Yearb Med Inform, vol. 95, no. 20, pp. 128-144, 2008.

[58] S. Sarawagi, "Information extraction," Foundations and trends in databases, vol. 1, no. 3, pp. 261-377, 2008.

[59] S. G. Small and L. Medsker, "Review of information extraction technologies and applications," Neural Computing and Applications, vol. 25, no. 3-4, pp. 533-548, 2014.

[60] A. Névéol, P. Zweigenbaum, S. Editors, and I. Yearbook, "Clinical Natural Language Processing in 2014: Foundational Methods Supporting Efficient Healthcare Topics in Clinical NLP," IMIA Yearbook, vol. 10, no. 1, pp. 194-198, 2015.

[61] A. Névéol and P. Zweigenbaum, "Clinical Natural Language Processing in 2015: Leveraging the Variety of Texts of Clinical Interest," IMIA Yearbook, no. 1, pp. 234-239, 2016.

[62] P. Spyns, "Natural Language Processing in Medicine: An Overview," Methods of Information in Medicine, vol. 35, no. 4-5, p. 9019092, 1996. 
[63] A. Névéol, H. K. Dalianis, G. Savova, and P. Zweigenbaum, "Clinical Natural Language Processing in languages other than English: opportunities and challenges," J Biomed Semantics, vol. 9, no. 12, pp. 1-13, 2018.

[64] W. W. Yim, M. Yetisgen, W. P. Harris, and W. K. Sharon, "Natural Language Processing in Oncology Review," JAMA Oncology, vol. 2, no. 6 , pp. 797-804, 2016.

[65] "PubMed." https://www.ncbi.nlm.nih.gov/pubmed/. [Accessed: 26Feb-2019].

[66] "MEDLINE." https://www.nlm.nih.gov/bsd/medline.html. [Accessed: 26-Feb-2019].

[67] "ACL Anthology." http://aclweb.org/anthology/. [Accessed: 26Feb-2019].

[68] "ACM Digital Library." https://dl.acm.org/. [Accessed: 26-Feb2019].

[69] "Springer - International Publisher Science, Technology, Medicine." https://www.springer.com/gp. [Accessed: 26-Feb-2019].

[70] P. Haug, S. Koehler, L. M. Lau, P. Wang, R. Rocha, and S. Huff, "A natural language understanding system combining syntactic and semantic techniques," in Proceedings of the Annual Symposium on Computer Application in Medical Care, pp. 247-51, 1994.

[71] P. J. Haug, S. Koehler, L. M. Lau, P. Wang, R. Rocha, and S. M. Huff, "Experience with a mixed semantic/syntactic parser," in Proceedings of the Annual Symposium on Computer Application n Medical Care., p. 284, 1995.

[72] M. Fiszman, P. J. Haug, and P. R. Frederick, "Automatic extraction of PIOPED interpretations from ventilation/perfusion lung scan reports," in Proc AMIA Symp, pp. 860-864, 1998.

[73] W. E. Trick, W. W. Chapman, M. F. Wisniewski, B. J. Peterson, S. L. Solomon, and R. A. Weinstein, "Electronic Interpretation of Chest Radiograph Reports to Detect Central Venous Catheters," Infection Control 83 Hospital Epidemiology, vol. 24, no. 12, pp. 950-954, 2003. 
[74] P. Zweigenbaum, "MENELAS: an access system for medical records using natural language," Computer Methods and Programs in Biomedicine, vol. 45, no. 1-2, pp. 117-120, 1994.

[75] C. Friedman, G. Hripcsak, W. DuMouchel, S. B. Johnson, and P. D. Clayton, "Natural language processing in an operational clinical information system," Natural Language Engineering, vol. 1, no. 01, pp. 83-108, 1995.

[76] C. Friedman, "Towards a Comprehensive Medical Language Processing System: Methods and Issues," in Proc AMIA Annu Fall Symp, pp. 595599, 1997.

[77] C. Friedman and G. Hripcsak, "Natural language processing and its future in medicine," Acad Med, vol. 74, no. 8, pp. 890-895, 1999.

[78] C. Friedman, C. Knirsch, L. Shagina, and G. Hripcsak, "Automating a Severity Score Guideline for Community-Acquired Pneumonia Employing Medical Language Processing of Discharge Summaries," in Proc AMIA Symp, pp. 256-260, 1999.

[79] C. Friedman, "A Broad-Coverage Natural Language Processing System," in Proc AMIA Symp, pp. 270-274, 2000.

[80] C. Friedman, H. Liu, L. Shagina, S. Johnson, and G. Hripcsak, "Evaluating the UMLS as a source of lexical knowledge for medical language processing," in AMIA Annual Symposium Proceedings, pp. 189-93, 2001.

[81] K. Haerian, H. Salmasian, and C. Friedman, "Methods for Identifying Suicide or Suicidal Ideation in EHRs," AMIA Annu Symp Proc, vol. 2012, pp. 1244-1253, 2012.

[82] E. E. Nelson and A. E. Guyer, "Detection of pharmacovigilance-related adverse events using electronic health records and automated methods," Clin Pharmacol Ther, vol. 92, no. 2, pp. 228-234, 2012.

[83] H. Salmasian, D. E Freedberg, J. A Abrams, and C. Friedman, "An automated tool for detecting medication overuse based on the electronic health records," Pharmacoepidemiol Drug Saf, vol. 22, no. 2, pp. 183189, 2013. 
[84] A. R. Aronson, "Effective mapping of biomedical text to the UMLS Metathesaurus: the MetaMap program.," in Proceedings of the AMIA Symposium, p. 17, 2001.

[85] A. R. Aronson, "MetaMap : Mapping Text to the UMLS Metathesaurus," tech. rep., 2006.

[86] L. Hj and B. Go, "Understanding and using the medical subject headings ( MeSH ) vocabulary to perform literature searches," JAMA, vol. 271, no. 14 , pp. 1103-1108, 1994.

[87] "Medical Subject Headings - Home Page." https://www.nlm.nih.gov/ mesh/. [Accessed: 26-Feb-2019].

[88] D. K.a, S. C.a, D. J.b, I. S. a. C, and F. J. C. a. C, "Identification of pneumonia and influenza deaths using the death certificate pipeline," BMC Medical Informatics and Decision Making, vol. 12, no. 1, 2012.

[89] C. A. Bejan, F. Xia, L. Vanderwende, M. M. Wurfe, and M. YetisgenYildiz, "Pneumonia identification using statistical feature selection," Journal of the American Medical Informatics Association, vol. 19, no. 5, pp. 817-823, 2012.

[90] M. Yetisgen-Yildiz, C. A. Bejan, L. Vanderwende, F. Xia, H. L. Evans, and M. M. Wurfel, "Automated Tools for Phenotype Extraction from Medical Records," AMIA Summits on Translational Science proceedings AMIA Summit on Translational Science, vol. 2013, no. December 2013, p. $283,2013$.

[91] A. V. Gundlapalli, A. Redd, M. Carter, G. Divita, S. Shen, M. Palmer, and M. H. Samore, "Validating a strategy for psychosocial phenotyping using a large corpus of clinical text," Journal of the American Medical Informatics Association, vol. 20, no. e2, pp. e355-e364, 2013.

[92] J. St-Maurice and M. H. Kuo, "Analyzing primary care data to characterize inappropriate emergency room use," Studies in Health Technology and Informatics, vol. 180, pp. 990-994, 2012.

[93] J. St-Maurice, M. H. Kuo, and P. Gooch, "A proof of concept for assessing emergency room use with primary care data and natural 
language processing," Methods of Information in Medicine, vol. 52, no. 1, pp. 33-42, 2013.

[94] H. Zhu, Y. Ni, C. Peng, Z. Qiu, and F. Cao, "Automatic extracting of patient-related attributes: Disease, age, gender and race," Studies in Health Technology and Informatics, vol. 180, no. 399, pp. 589-593, 2012.

[95] C. Thorne, E. Cardillo, C. Eccher, M. Montali, and D. Calvanese, "Process fragment recognition in clinical documents," in Congress of the Italian Association for Artificial Intelligence, vol. 8249 LNAI, pp. 227238, 2013.

[96] R. Khare, J. Li, and Z. Lu, "LabeledIn: Cataloging labeled indications for human drugs," Journal of Biomedical Informatics, vol. 52, pp. 448$456,2014$.

[97] L. M. Christensen, P. J. Haug, and M. Fiszman, "MPLUS: A Probabilistic Medical Language Understanding System The M + Semantic Model," in Proceedings of the ACL-02 workshop on Natural language processing in the biomedical domain, no. July, pp. 29-36, 2002.

[98] J. C. Denny, P. R. Irani, F. H. Wehbe, J. D. Smithers, A. Spickard 3rd, J. C. Denny, P. R. Irani, F. H. Wehbe, J. D. Smithers, and A. Spickard 3rd, "The KnowledgeMap project: development of a conceptbased medical school curriculum database," AMIA 2003 Symposium Proceedings, vol. Annual Sym, pp. 195-199, 2003.

[99] "KMCI - KnowledgeMap Concept Indexer Center for Precision Medicine." https://www vumc.org/cpm/center-precision-medicine-blog/ kmci-knowledgemap-concept-indexer. [Accessed: 26-Feb-2019].

[100] G. K. Savova, J. J. Masanz, P. V. Ogren, J. Zheng, S. Sohn, K. C. KipperSchuler, and C. G. Chute, "Mayo clinical Text Analysis and Knowledge Extraction System (cTAKES): Architecture, component evaluation and applications," Journal of the American Medical Informatics Association, vol. 17, no. 5, pp. 507-513, 2010. 
[101] "Apache $\{\mathrm{cTAKES}\}^{\mathrm{TM}}$ - clinical Text Analysis Knowledge Extraction System." https://researcher.watson.ibm.com/researcher/ view\{_\}group.php?id=3259. [Accessed: 26-Feb-2019].

[102] "Apache OpenNLP." https://opennlp.apache.org/. [Accessed: 26Feb-2019].

[103] S. Sohn and G. K. Savova, "Mayo clinic smoking status classification system: extensions and improvements," AMIA ... Annual Symposium proceedings. AMIA Symposium, vol. 2009, pp. 619-623, 2009.

[104] I. J. Kullo, J. Fan, J. Pathak, G. K. Savova, Z. Ali, and C. G. Chute, "Leveraging informatics for genetic studies: use of the electronic medical record to enable a genome-wide association study of peripheral arterial disease," J Am Med Inform Assoc, vol. 5, pp. 568-574, 2010.

[105] M. Liu, A. Shah, M. Jiang, N. B. Peterson, M. C. Aldrich, Q. Chen, E. A. Bowton, H. Liu, J. C. Denny, and H. Xu, "A Study of Transportability of an Existing Smoking Status Detection Module across Institutions," AMIA Annu Symp Proc, vol. 2012, pp. 577-586, 2012.

[106] R. Khor, W. Yip, M. Bressel, W. Rose, G. Duchesne, and F. Foroudi, "Automated smoking status extraction from free text: adapting a system for use in the Australian context," J. Med. Imag. Radiat. Oncol., vol. 57, no. 148, p. 2019, 2013.

[107] R. Khor, W.-k. Yip, M. Bressel, W. Rose, G. Duchesne, and F. Foroudi, "Practical implementation of an existing smoking detection pipeline and reduced support vector machine training corpus requirements," . J Am Med Inform Assoc, vol. 21, no. 1, pp. 27-30, 2014.

[108] A. Khalifa and S. Meystre, "Adapting existing natural language processing resources for cardiovascular risk factors identification in clinical notes," Journal of Biomedical Informatics, vol. 58, pp. S128-S132, 2015.

[109] S. Sohn, J.-p. A. Kocher, C. G. Chute, and G. K. Savova, "Drug side effect extraction from clinical narratives of psychiatry and psychology patients," J Am Med Inform Assoc, vol. 18, pp. 144-149, 2011.

[110] L. Deleger, H. Brodzinski, H. Zhai, Q. Li, T. Lingren, E. S. Kirkendall, E. Alessandrini, and I. Solti, "Developing and evaluating an automated 
appendicitis risk stratification algorithm for pediatric patients in the emergency department," Journal of the American Medical Informatics Association, vol. 20, no. E2, pp. e212-e220, 2013.

[111] V. Kumar, K. Liao, S.-c. Cheng, S. Yu, U. Kartoun, A. Brettman, V. Gainer, A. Cagan, S. Murphy, G. Savova, P. Chen, P. Szolovits, Z. Xia, E. Karlson, R. Plenge, A. Ananthakrishnan, S. Churchill, T. Cai, I. Kohane, and S. Shaw, "NATURAL LANGUAGE PROCESSING IMPROVES PHENOTYPIC ACCURACY IN AN ELECTRONIC MEDICAL RECORD COHORT OF TYPE 2 DIABETES AND CARDIOVASCULAR DISEASE," Journal of the American College of Cardiology, vol. 63, no. 12, p. A1359, 2014.

[112] C. Lin, D. Dligach, T. A. Miller, S. Bethard, and G. K. Savova, "Multilayered temporal modeling for the clinical domain," Journal of the American Medical Informatics Association, vol. 23, no. 2, pp. 387-395, 2016.

[113] H. Hochheiser, M. Castine, D. Harris, G. Savova, and R. S. Jacobson, "An information model for computable cancer phenotypes," BMC Medical Informatics and Decision Making, vol. 16, no. 1, pp. 1-15, 2016.

[114] M. Torii, Z. Hu, C. H. Wu, and H. Liu, "BioTagger-GM: A Gene/Protein Name Recognition System," Journal of the American Medical Informatics Association, vol. 16, no. 2, pp. 247-255, 2009.

[115] M. Torii, K. Wagholikar, and H. Liu, "Using machine learning for concept extraction on clinical documents from multiple data sources," Journal of the American Medical Informatics Association, vol. 18, no. 5, pp. 580-587, 2011.

[116] "MedTagger." http://ec2-184-73-168-219.compute-1.amazonaws. com/index.php/MedTagger. [Accessed: 26-Feb-2019].

[117] H. Liu, K. Wagholikar, S. Jonnalagadda, and S. Sohn, "Integrated cTAKES for concept mention detection and normalization," CEUR Workshop Proceedings, vol. 1179, 2013.

[118] H. Xu, S. P. Stenner, S. Doan, K. B. Johnson, L. R. Waitman, and J. C. Denny, "MedEx: A medication information extraction system 
for clinical narratives," Journal of the American Medical Informatics Association, vol. 17, no. 1, pp. 19-24, 2010.

[119] L. W. D'Avolio, T. M. Nguyen, and L. D. Fiore, "The automated retrieval console (ARC): open source software for streamlining the process of natural language processing," Proceedings of the 1st ACM International Health Informatics Symposium, pp. 469-473, 2010.

[120] L. W. D'Avolio, T. M. Nguyen, W. R. Farwell, Y. Chen, F. Fitzmeyer, O. M. Harris, and L. D. Fiore, "Evaluation of a generalizable approach to clinical information retrieval using the automated retrieval console (ARC)," Journal of the American Medical Informatics Association, vol. 17, no. 4, pp. 375-382, 2010.

[121] "ARC_ Automated Retrieval Console _ BLUlab." http://blulab.chpc utah.edu/content/arc-automated-retrieval-console. [Accessed: 26-Feb-2019].

[122] L. W. D'Avolio, T. M. Nguyen, S. Goryachev, and L. D. Fiore, "Automated concept-level information extraction to reduce the need for custom software and rules development," Journal of the American Medical Informatics Association, vol. 18, no. 5, pp. 607-613, 2011.

[123] Q. T. Zeng, S. Goryachev, S. Weiss, M. Sordo, S. N. Murphy, and R. Lazarus, "Extracting principal diagnosis, co-morbidity and smoking status for asthma research: Evaluation of a natural language processing system," BMC Medical Informatics and Decision Making, vol. 6, pp. 1-9, 2006.

[124] "HITEx Manual." https://www.i2b2.org/software/projects/ hitex/hitex_manual.html. [Accessed: 26-Feb-2019].

[125] G. Fette, M. Ertl, A. Wörner, P. Kluegl, S. Störk, and F. Puppe, "Information Extraction from Unstructured Electronic Health Records and Integration into a Data Warehouse," GI-Jahrestagung, vol. LNI 208, pp. 1237-1251, 2012.

[126] M. Taboada, M. Meizoso, D. Martínez, D. Riaño, and A. Alonso, "Combining open-source natural language processing tools to parse clinical practice guidelines," Expert Systems, vol. 30, no. 1, pp. 3-11, 2013. 
[127] "The Stanford Parser: A statistical parser." https://nlp.stanford edu/software/lex-parser.shtml. [Accessed: 26-Feb-2019].

[128] "Semantic Knowledge Representation." https://semrep.nlm.nih. gov/. [Accessed: 26-Feb-2019].

[129] S. Doan, M. Conway, T. M. Phuong, and L. Ohno-Machado, "Natural Language Processing in Biomedicine: A Unified System Architecture Overview," Clinical Bioinformatics, 2Nd Edition, vol. 1168, pp. 275-294, 2014.

[130] S. R. Halgrim, H. Liu, S. Sohn, C. Clark, S. P. Murphy, and C. G. Chute, "MedXN: an open source medication extraction and normalization tool for clinical text," Journal of the American Medical Informatics Association, vol. 21, no. 5, pp. 858-865, 2014.

[131] "MedXN." http://ohnlp.org/index.php/MedXN. [Accessed: 26-Feb2019].

[132] E. Soysal, J. Wang, M. Jiang, Y. Wu, S. Pakhomov, H. Liu, and H. Xu, "CLAMP - a toolkit for efficiently building customized clinical natural language processing pipelines," Journal of the American Medical Informatics Association, vol. 25, no. 3, pp. 331-336, 2018.

[133] "CLAMP - Natural Language Processing (NLP) Software." https: //clamp.uth.edu/, [Accessed: 26-Feb-2019].

[134] I. McCowan, D. Moore, and M.-J. Fry, "Automated Cancer Stage Classification from Free-text Histology Reports," in Conf Proc IEEE Eng Med Biol Soc, pp. 5153-5156, 2006.

[135] A. Coden, G. Savova, I. Sominsky, M. Tanenblatt, J. Masanz, K. Schuler, J. Cooper, W. Guan, and P. C. de Groen, "Automatically extracting cancer disease characteristics from pathology reports into a Disease Knowledge Representation Model," Journal of Biomedical Informatics, vol. 42, no. 5, pp. 937-949, 2009.

[136] D. A. Hanauer, G. Miela, A. M. Chinnaiyan, A. E. Chang, and D. W. Blayney, "The Registry Case Finding Engine: An Automated Tool to Identify Cancer Cases from Unstructured, Free-Text Pathology Reports and Clinical Notes," J Am Coll Surg, vol. 205, no. 5, pp. 690-697, 2007. 
[137] R. S. Crowley, M. Castine, K. Mitchell, G. Chavan, T. Mcsherry, and M. Feldman, "caTIES : a grid based system for coding and retrieval of surgical pathology reports and tissue specimens in support of translational research," J Am Med Inform Assoc, vol. 17, no. 3, pp. 253-264, 2010 .

[138] A. A. Thomas, C. Zheng, H. Jung, A. Chang, B. Kim, J. Gelfond, J. Slezak, K. Porter, S. J. Jacobsen, and G. W. Chien, "Extracting data from electronic medical records: Validation of a natural language processing program to assess prostate biopsy results," World Journal of Urology, vol. 32, no. 1, pp. 99-103, 2014.

[139] "Medical Text and Image Analytics - IBM." https://researcher. watson.ibm.com/researcher/view\{_\}group.php?id=3259. [Accessed: 26-Feb-2019].

[140] "caTIES - NCIP - caTissue Tools - National Cancer Institute - Confluence Wiki." https://wiki.nci.nih.gov/display/caTissue/caTIES. [Accessed: 26-Feb-2019].

[141] R. Costumero, A. Garcia-Pedrero, I. Sánchez, C. Gonzalo, and E. Menasalvas, "Electronic Health Records Analytics: Natural Language Processing and Image Annotation," in International Workshop on Big Data Applications and Principles, pp. 1-21, 2014.

[142] "Savana - Transform your clinical records free text into Big Data."

[143] L. Zhou, C. Friedman, S. Parsons, and G. Hripcsak, "System architecture for temporal information extraction, representation and reasoning in clinical narrative reports.," in AMIA ... Annual Symposium proceedings / AMIA Symposium. AMIA Symposium, no. Figure 1, pp. 869-873, 2005.

[144] B. M. Sundheim, "The message understanding conferences," in Proceedings of a workshop on held at Vienna, Virginia May 6-8, 1996 -, vol. 90, p. 35, 1996.

[145] "ACE Time Normalization (TERN) 2004 English Training Data v 1." https://catalog.ldc.upenn.edu/LDC2005T07. [Accessed: 18-Mar2019]. 
[146] "The ACE 2005 ( ACE05 ) Evaluation Plan Evaluation of the Detection and Recognition of ACE." https://pdfs.semanticscholar org/3a9b/136ca1ab91592df36f148ef16095f74d009e.pdf. [Accessed: 18-Mar-2019].

[147] "Recognition and Normalization of Time Expressions: ITCirst at TERN 2004." http://www.lsi.upc.edu/\{ \}nlp/meaning/ documentation/3rdYear/WP3.6.pdf. [Accessed: 18-Mar-2019].

[148] P. Mazur and R. Dale, "The DANTE temporal expression tagger," in Lecture Notes in Computer Science, vol. 5603, pp. 245-257, 2009.

[149] R. Ingria, R. Sauri, J. Pustejovsky, R. Gaizauskas, A. Setzer, G. Katz, D. Radev, and J. Castano, "TimeML: Robust Specification of Event and Temporal Expressions in Text," New directions in question answering, vol. 3, pp. 28-34, 2003.

[150] J. Pustejovsky, A. See, A. Setzer, B. Sundheim, D. Day, and M. Lazo, "The TimeBank corpus," In Corpus Linguistics, vol. 647-656, 2003.

[151] M. Verhagen, R. Gaizauskas, F. Schilder, M. Hepple, G. Katz, and J. Pustejovsky, "SemEval-2007 Task 15 : TempEval Temporal Relation Identification," in SemEval-2007 Task 15: TempEval Temporal Relation Identification, no. June, pp. 75-80, 2007.

[152] M. Verhagen, R. Saur I, T. Caselli, and J. Pustejovsky, "SemEval2010 Task 13 : TempEval-2," in Proceedings of the 5th International Workshop on Semantic Evaluation, no. July, pp. 57-62, 2010.

[153] N. UzZaman, H. Llorens, L. Derczynski, J. Allen, M. Verhagen, J. Pustejovsky, J. Allen, and J. Pustejovsky, "SemEval-2013 Task 1: TempEval3: Evaluating Time Expressions, Events, and Temporal Relations," in Second Joint Conference on Lexical and Computational Semantics ( $\left.{ }^{*} S E M\right)$, Volume 2: Proceedings of the Seventh International Workshop on Semantic Evaluation (SemEval 2013), vol. 2, pp. 1-9, 2013.

[154] M. T. Vicente-díez, J. M. Schneider, and P. Martínez, "UC3M system : Determining the Extent, Type and Value of Time Expressions in TempEval-2," in SemEval '10 Proceedings of the 5th International Workshop on Semantic Evaluation, no. July, pp. 329-332, 2010. 
[155] J. Str and M. Gertz, "HeidelTime: High Quality Rule-based Extraction and Normalization of Temporal Expressions," in SemEval '10 Proceedings of the 5th International Workshop on Semantic Evaluation, no. July, pp. 321-324, 2010.

[156] N. UzZaman and J. F. Allen, "TRIPS and TRIOS System for TempEval2: Extracting Temporal Information from Text," in Proceedings of the 5th International Workshop on Semantic Evaluation (SemEval 2010), no. July, pp. 276-283, 2010.

[157] M. Verhagen and J. Pustejovsky, "Temporal processing with the TARSQI toolkit," in Proc. of the 22nd International Conference on on Computational Linguistics (COLING'08), no. August, pp. 189-192, 2008.

[158] I. Mani, "Recent Developments in Temporal Information Extraction," in Proceedings of the International Conference on Recent Advances in Natural Language Processing (RANLP'03), pp. 45-60, 2004.

[159] I. Mani and G. Wilson, "Robust temporal processing of news," in Proceedings of the 38th Annual Meeting on Association for Computational Linguistics - ACL '00, no. Muc 1998, pp. 69-76, 2000.

[160] M. Filannino and G. Nenadic, "Temporal expression extraction with extensive feature type selection and a posteriori label adjustment," Data and Knowledge Engineering, vol. 100, pp. 19-33, 2015.

[161] Q. Ning, B. Zhou, Z. Feng, H. Peng, and D. Roth, "CogCompTime: A Tool for Understanding Time in Natural Language," in Proceedings of the 2018 Conference on Empirical Methods in Natural Language Processing: System, pp. 72-77, 2018.

[162] X. Zhong, A. Sun, and E. Cambria, "Time Expression Analysis and Recognition Using Syntactic Token Types and General Heuristic Rules," in Proceedings of the 55th Annual Meeting of the Association for Computational Linguistics (Volume 1: Long Papers), pp. 420-429, 2017.

[163] W. Sun, A. Rumshisky, and O. Uzuner, "Evaluating temporal relations in clinical text: 2012 i2b2 Challenge," Journal of the American Medical Informatics Association, vol. 20, no. 5, pp. 806-813, 2013. 
[164] S. Bethard, L. Derczynski, G. Savova, J. Pustejovsky, and M. Verhagen, "SemEval-2015 Task 6: Clinical TempEval," in Proceedings of the 9th International Workshop on Semantic Evaluation (SemEval 2015), no. SemEval, pp. 806-814, 2015.

[165] S. Bethard, G. Savova, W.-T. Chen, L. Derczynski, J. Pustejovsky, and M. Verhagen, "SemEval-2016 Task 12: Clinical TempEval," in Proceedings of the 10th International Workshop on Semantic Evaluation (SemEval-2016), pp. 1052-1062, 2016.

[166] S. Bethard, G. Savova, M. Palmer, and J. Pustejovsky, "SemEval-2017 Task 12: Clinical TempEval," in Proceedings of the 11th International Workshop on Semantic Evaluation (SemEval-2017), pp. 565-572, 2017.

[167] W. Sun, A. Rumshisky, and O. Uzuner, "Annotating temporal information in clinical narratives," Journal of Biomedical Informatics, vol. 46, no. SUPPL., pp. S5-S12, 2013.

[168] W. F. S. IV, S. Bethard, S. Finan, M. Palmer, S. Pradhan, P. C. de Groen, B. Erickson, T. Miller, C. Lin, G. Savova, and J. Pustejovsky, "Temporal Annotation in the Clinical Domain," Transactions of the Association for Computational Linguistics, vol. 2, no. 1, pp. 143-154, 2014.

[169] S. Velupillai, D. L. Mowery, S. Abdelrahman, L. Christensen, and W. W. Chapman, "BluLab: Temporal Information Extraction for the 2015 Clinical TempEval Challenge," in Proceedings of the 9th International Workshop on Semantic Evaluation (SemEval 2015), no. SemEval, pp. 815-819, 2015.

[170] H.-J. Lee, H. Xu, J. Wang, Y. Zhang, S. Moon, J. Xu, and Y. Wu, "UTHealth at SemEval-2016 Task 12: an End-to-End System for Temporal Information Extraction from Clinical Notes," in Proceedings of the 10th International Workshop on Semantic Evaluation (SemEval-2016), pp. 1292-1297, 2016.

[171] A. Leeuwenberg and M.-F. Moens, "KULeuven-LIIR at SemEval-2017 Task 12: Cross-Domain Temporal Information Extraction from Clinical Records," in Proceedings of the 11th International Workshop on Semantic Evaluation (SemEval-2017), no. Phase I, pp. 1030-1034, 2017. 
[172] S. MacAvaney, A. Cohan, and N. Goharian, "GUIR at SemEval-2017 Task 12: A Framework for Cross-Domain Clinical Temporal Information Extraction," in Proceedings of the 11th International Workshop on Semantic Evaluation (SemEval-2017), pp. 1021-1026, 2017.

[173] G. Savova, S. Bethard, W. Styler, J. Martin, M. Palmer, J. Masanz, and W. Ward, "Towards temporal relation discovery from the clinical narrative.," in AMIA ... Annual Symposium proceedings / AMIA Symposium. AMIA Symposium, vol. 2009, pp. 568-572, 2009.

[174] "cTAKES 4.0 - Temporal Module." https://cwiki.apache.org/ confluence/display/CTAKES/cTAKES+4.0+-+Temporal+Module. [Accessed: 01-Mar-2019].

[175] T. Hao, X. Pan, Z. Gu, Y. Qu, and H. Weng, "A pattern learning-based method for temporal expression extraction and normalization from multi-lingual heterogeneous clinical texts," BMC Medical Informatics and Decision Making, vol. 18, no. Suppl 1, 2018.

[176] "Stanford CoreNLP - Natural language software." https:// stanfordnlp.github.io/CoreNLP/, [Accessed: 27-Feb-2019].

[177] P. Raghavan, E. Fosler-lussier, and A. M. Lai, "Temporal Classification of Medical Events," in Proceedings of the 2012 Workshop on Biomedical Natural Language Processing, no. BioNLP, pp. 29-37, 2012.

[178] D. R. Radev, "A Common Theory of Information Fusion from Multiple Text Sources; Step One: Cross-Document Structure," in Proceedings of the 1st SIGdial Workshop on Discourse and Dialogue, pp. 74-83, 2000.

[179] L. Zhou and G. Hripcsak, "Temporal reasoning with medical data-A review with emphasis on medical natural language processing," Journal of Biomedical Informatics, vol. 40, no. 2, pp. 183-202, 2007.

[180] "Event." https://glossary.sil.org/term/event. [Accessed: 10Mar-2019].

[181] A. Lincoln, J. Kim, D. Davidson, D. Lewis, P.-e. Account, C. Object, E. Fine-grainedness, T. O. Line, T. F. Position, C. Object, T. C. Criterion, T. S. Criterion, O. Commitment, and D. Lewis, "Events," 2018. 
[182] A. Jagannatha and H. Yu, "Bidirectional Recurrent Neural Networks for Medical Event Detection in Electronic Health Records," in In proceedings of NAACL HLT 2016, 2016.

[183] Daniel Jurafsky and James H. Martin, Speech and Language Processing: An Introduction to Natural Language Processing, Computational Linguistics, and Speech Recognition, vol. 26. 2000.

[184] C. Lin, D. Dligach, T. A. Miller, S. Bethard, and G. K. Savova, "Multilayered temporal modeling for the clinical domain," Journal of the American Medical Informatics Association, vol. 23, no. 2, pp. 387-395, 2016.

[185] C. Combi and Y. Shahar, "Temporal reasoning and temporal data maintenance in medicine: Issues and challenges," Computers in Biology and Medicine, vol. 27, no. 5, pp. 353-368, 1997.

[186] J. F. Fries, "Time-Oriented Patient Records and a Computer Databank," JAMA: The Journal of the American Medical Association, vol. 222, no. 12 , pp. 1536-1542, 1972.

[187] B. Buchanan and E. Shortliffe, Rule-Based Expert Systems: The MYCIN Experiments of the Stanford Heuristic Programming Project. 1984.

[188] E. H. Shortliffe, C. A. Scott, M. B. Bischoff, B. A. Campbell, W. Van Melle, and C. D. Jacobs, "ONCOCIN: An expert system for Oncology protocol management," in Proceedings of the 7th International Joint Conference on Artificial Intelligence, vol. 2, pp. 876-881, 1981.

[189] C. S. Jensen, S. Member, and R. T. Snodgrass, "Temporal Data Management," IEEE Transactions on Knowledge and data Engineering, vol. 11, no. 1, pp. 36-44, 1999.

[190] M. H. Böhlen, "Temporal database system implementations," Sigmod Record, vol. 24, no. 4, pp. 53-60, 1995.

[191] T. B. Pedersen and C. S. Jensen, "Research issues in clinical data warehousing," in Scientific and Statistical Database Management, 1998. Proceedings. Tenth International Conference on, pp. 43-52, 1998. 
[192] Y. Shahar, "Timing is everything: Temporal reasoning and temporal data maintenance in medicine," in Joint European Conference on Artificial Intelligence in Medicine and Medical Decision Making, pp. 30-46, 1999.

[193] G. Hripcsak, N. D. Soulakis, L. Li, F. P. Morrison, A. M. Lai, C. Friedman, N. S. Calman, and F. Mostashari, "Syndromic Surveillance Using Ambulatory Electronic Health Records," Journal of the American Medical Informatics Association, vol. 16, no. 3, pp. 354-361, 2009.

[194] D. McDermott, "A temporal logic for reasoning about processes and plans," Cognitive Science, vol. 6, no. 2, pp. 101-155, 1982.

[195] T. L. Dean and D. V. McDermott, "Temporal data base management," Artificial Intelligence, vol. 32, no. 1, pp. 1-55, 1987.

[196] J. F. Allen, "Maintaining knowledge about temproal intervals," Readings in qualitative reasoning about physical systems, vol. 26, no. 11, pp. 361372, 1990.

[197] K. Kahn and G. A. Gorry, "Mechanizing temporal knowledge," Artificial Intelligence, vol. 9, no. 1, pp. 87-108, 1977.

[198] A. Bochman, "Concerted Instant-lnterval Temporal Semantics I : Temporal Ontologies," Notre Dame Journal of Formal Logic, vol. 31, no. 3, pp. 403-414, 1990.

[199] L. Vila and E. Schwalb, "A Theory of Time and Temporal Incidence based on Instants and Periods," in Proc.International Workshop on Temporal Representation and Reasoning, pp. 21-28, 1996.

[200] N. V. Findler and D. Chen, "On the problems of time retrieval of temporal relations causality, and coexistence," International Journal of Computer \& Information Sciences, vol. 2, no. 3, pp. 161-185, 1973.

[201] W. Sun, A. Rumshisky, and O. Uzuner, "Temporal reasoning over clinical text: The state of the art," Journal of the American Medical Informatics Association, vol. 20, no. 5, pp. 814-819, 2013.

[202] A. K. Das and M. A. Musen, "A comparison of the temporal expressiveness of three database query methods," in Proceedings / the Annual 
Symposium on Computer Application [sic] in Medical Care Symposium on Computer Applications in Medical Care, pp. 331-337, 1995.

[203] M. Kahn, L. Fagan, and S. Tu, "Extensions to the time-oriented database model to support temporal reasoning in medical expert systems," Methods of Information in Medicine, vol. 30, no. 1, pp. 4-14, 1991.

[204] R. Schmidt, S. Ropele, C. Enzinger, K. Petrovic, S. Smith, H. Schmidt, P. M. Matthews, and F. Fazekas, "White matter lesion progression, brain atrophy, and cognitive decline: The Austrian stroke prevention study," Annals of Neurology, vol. 58, no. 4, pp. 610-616, 2005.

[205] G. K. Savova, J. E. Olson, S. P. Murphy, V. L. Cafourek, F. J. Couch, M. P. Goetz, J. N. Ingle, V. J. Suman, C. G. Chute, and R. M. Weinshilboum, "Automated discovery of drug treatment patterns for endocrine therapy of breast cancer within an electronic medical record," Journal of the American Medical Informatics Association, vol. 19, no. E1, pp. 83-89, 2012.

[206] L. Hirschman, "Retrieving Time Information From Natural-Language Texts," in 80 Proceedings of the 3rd annual ACM conference on Research and development in information retrieval, pp. 154-171, 1980.

[207] N. Sager, "Syntactic Analysis of Natural Language," Advances in Computers, vol. 8, pp. 153-188, 1967.

[208] N. Sager, "Natural Language Analysis and Processing," Encyclopedia of Computer Science and Technology, vol. 11, pp. 152-169, 1978.

[209] L. Hirschman, R. Grishman, and N. Sager, "From text to structured information: automatic processing of medical reports," in Proceedings of the June 7-10, 1976, AFIPS Conference Proceedings 45, pp. 267-275, 1976.

[210] G. Story and L. Hirschman, "Data Base Design for Natural Language Medical Data," Journal of Medical Systems, vol. 6, no. 1, pp. 77-88, 1982. 
[211] K. K. Obermeier, "Klaus K. Obermeier Battelle's Columbus Laboratories," in Proceedings of the 1986 ACM fourteenth annual conference on Computer science, pp. 331-339, 1986.

[212] TimeML Working Group, "Guidelines for Temporal Expression Annotation for English for TempEval 2010," 2009.

[213] B. Boguraev, J. Pustejovsky, R. Ando, and M. Verhagen, "TimeBank evolution as a community resource for TimeML parsing," Language Resources and Evaluation, vol. 41, no. 1, pp. 91-115, 2007.

[214] M. Verhagen, R. Gaizauskas, F. Schilder, M. Hepple, J. Moszkowicz, and J. Pustejovsky, "The tempEval challenge: Identifying temporal relations in text," Language Resources and Evaluation, vol. 43, no. 2, pp. 161-179, 2009.

[215] H. Jung, J. Allen, N. Blaylock, W. D. Beaumont, L. Galescu, and M. Swift, "Building timelines from narrative clinical records: initial results based-on deep natural language understanding," in Proceedings of BioNLP 2011 Workshop, pp. 146-154, 2011.

[216] A. Nikfarjam, E. Emadzadeh, and G. Gonzalez, "Towards generating a patient's timeline: Extracting temporal relationships from clinical notes," Journal of Biomedical Informatics, vol. 46, no. SUPPL., pp. S40-S47, 2013.

[217] G. Moharasan and T.-B. Ho, "Extraction of Temporal Information from Clinical Narratives," Journal of Healthcare Informatics Research, pp. 1-12, 2019.

[218] G. Hripcsak, L. Zhou, S. Parsons, A. K. Das, and S. B. Johnson, "Modeling electronic discharge summaries as a simple temporal constraint satisfaction problem," Journal of the American Medical Informatics Association, vol. 12, no. 1, pp. 55-63, 2005.

[219] L. Zhou, G. B. Melton, S. Parsons, and G. Hripcsak, "A temporal constraint structure for extracting temporal information from clinical narrative," Journal of Biomedical Informatics, vol. 39, no. 4, pp. 424439, 2006. 
[220] L. Zhou, S. Parsons, and G. Hripcsak, "The Evaluation of a Temporal Reasoning System in Processing Clinical Discharge Summaries," Journal of the American Medical Informatics Association, vol. 15, no. 1, pp. 99$106,2008$.

[221] H. Tissot, G. Gorrell, A. Roberts, L. Derczynski, M. Didonet, and D. Fabro, "UFPRSheffield : Contrasting Rule-based and Support Vector Machine Approaches to Time Expression Identification in Clinical TempEval," in Proceedings of the 9th International Workshop on Semantic Evaluation (SemEval 2015), no. SemEval, pp. 835-839, 2015.

[222] J. A. Fries, "Brundlefly at SemEval-2016 Task 12: Recurrent Neural Networks vs. Joint Inference for Clinical Temporal Information Extraction," in Proceedings of the 10th International Workshop on Semantic Evaluation (SemEval-2016), pp. 1274-1279, 2016.

[223] R. V. Chikka, "CDE-IIITH at SemEval-2016 Task 12: Extraction of Temporal Information from Clinical documents using Machine Learning techniques," in Proceedings of the 10th International Workshop on Semantic Evaluation (SemEval-2016), pp. 1237-1240, 2016.

[224] C. Hansart, D. De Meyere, P. Watrin, A. Bittar, and C. Fairon, "CENTAL at SemEval-2016 Task 12: a linguistically fed CRF model for medical and temporal information extraction," in Proceedings of the 10th International Workshop on Semantic Evaluation (SemEval-2016), pp. 1286-1291, 2016.

[225] A. Cohan, K. Meurer, and N. Goharian, "GUIR at SemEval-2016 task 12: Temporal Information Processing for Clinical Narratives," in Proceedings of the 10th International Workshop on Semantic Evaluation (SemEval-2016), pp. 1248-1255, 2016.

[226] S. P. R., M. R., and Y. Niwa, "Hitachi at SemEval-2016 Task 12: A Hybrid Approach for Temporal Information Extraction from Clinical Notes," in Proceedings of the 10th International Workshop on Semantic Evaluation (SemEval-2016), vol. 2015, pp. 1231-1236, 2016.

[227] A. Leeuwenberg and M.-F. Moens, "KULeuven-LIIR at SemEval 2016 Task 12: Detecting Narrative Containment in Clinical Records," in 
Proceedings of the 10th International Workshop on Semantic Evaluation (SemEval-2016), pp. 1280-1285, 2016.

[228] C. Grouin and V. MORICEAU, "LIMSI at SemEval-2016 Task 12: machine-learning and temporal information to identify clinical events and time expressions," in Proceedings of the 10th International Workshop on Semantic Evaluation (SemEval-2016), pp. 1225-1230, 2016.

[229] J. Tourille, O. Ferret, A. Névéol, and X. Tannier, "LIMSI-COT at SemEval-2016 Task 12: Temporal relation identification using a pipeline of classifiers," in Proceedings of the 10th International Workshop on Semantic Evaluation (SemEval-2016), pp. 1136-1142, 2016.

[230] M. Barros, A. Lamurias, M. Antunes, J. Teixeira, A. Pinheiro, and F. M. Couto, "ULISBOA at SemEval-2016 Task 12 : Extraction of temporal expressions , clinical events and relations using IBEnt," in Proceedings of the 10th International Workshop on Semantic Evaluation (SemEval 2016), pp. 1263-1267, 2016.

[231] A. AAl Abdulsalam, S. Velupillai, and S. Meystre, "UtahBMI at SemEval-2016 Task 12: Extracting Temporal Information from Clinical Text," in Proceedings of the 10th International Workshop on Semantic Evaluation (SemEval-2016), pp. 1256-1262, 2016.

[232] P. Li and H. Huang, "UTA DLNLP at SemEval-2016 Task 12: Deep Learning Based Natural Language Processing System for Clinical Information Identification from Clinical Notes and Pathology Reports," in Proceedings of the 10th International Workshop on Semantic Evaluation (SemEval-2016), pp. 1268-1273, 2016.

[233] T. Caselli and R. Morante, "VUACLTL at SemEval 2016 Task 12: A CRF Pipeline to Clinical TempEval," in Proceedings of the 10th International Workshop on Semantic Evaluation (SemEval-2016), pp. 12411247, 2016.

[234] S. P. R., M. R., and Y. Niwa, "Hitachi at SemEval-2017 Task 12: System for temporal information extraction from clinical notes," in Proceedings of the 11th International Workshop on Semantic Evaluation (SemEval-2017), pp. 1005-1009, 2017. 
[235] J. Tourille, O. Ferret, X. Tannier, and A. Névéol, "LIMSI-COT at SemEval-2017 Task 12: Neural Architecture for Temporal Information Extraction from Clinical Narratives," in Proceedings of the 11th International Workshop on Semantic Evaluation (SemEval-2017), pp. 597-602, 2017.

[236] P.-Y. Huang, H.-H. Huang, Y.-W. Wang, C. Huang, and H.-H. Chen, "NTU-1 at SemEval-2017 Task 12: Detection and classification of temporal events in clinical data with domain adaptation," in Proceedings of the 11th International Workshop on Semantic Evaluation (SemEval-2017), pp. 1010-1013, 2017.

[237] A. Lamurias, D. Sousa, S. Pereira, L. Clarke, and F. M. Couto, "ULISBOA at SemEval-2017 Task 12: Extraction and classification of temporal expressions and events," in Proceedings of the 11th International Workshop on Semantic Evaluation (SemEval-2017), pp. 1019-1023, 2017.

[238] Y. Long, Z. Li, X. Wang, and C. Li, "XJNLP at SemEval-2017 Task 12: Clinical temporal information ex-traction with a Hybrid Model," in Proceedings of the 11th International Workshop on Semantic Evaluation (SemEval-2017), no. 2016, pp. 1014-1018, 2017.

[239] H. J. Lee, Y. Zhang, M. Jiang, J. Xu, C. Tao, and H. Xu, "Identifying direct temporal relations between time and events from clinical notes," BMC Medical Informatics and Decision Making, vol. 18, no. Suppl 2, pp. 23-34, 2018.

[240] M. Miwa and M. Bansal, "End-to-End Relation Extraction using LSTMs on Sequences and Tree Structures," in Proceedings of the 54th Annual Meeting of the Association for Computational Linguistics, pp. 1105-1116, 2016.

[241] D. Galvan, N. Okazak, K. Matsud, and K. Inui, "Investigating the Challenges of Temporal Relation Extraction from Clinical Text," in Proceedings of the Ninth International Workshop on Health Text Mining and Information Analysis, pp. 55-64, 2018.

[242] A. Naik, C. Bogart, and C. Rose, "Extracting Personal Medical Events for User Timeline Construction using Minimal Supervision," pp. 356$364,2017$. 
[243] "Ravelry; A Knit \& Crochet Community." http://www.ravelry.com. [Accessed: May-01-2020].

[244] "Breastcancer.org - breast cancer information and support." https: //www.breastcancer.org/. [Accessed: May-01-2020].

[245] S. Jeblee and G. Hirst, "Listwise temporal ordering of events in clinical notes," in In Proceedings of the Ninth International Workshop on Health Text Mining and Information AnalysisAnalysis, pp. 177-182, 2018.

[246] "GitHub - shiba24_learning2rank_ Learning to rank with neuralnet - RankNet and ListNet." https://github.com/shiba24/ learning2rank. [Accessed: May-01-2020].

[247] A.-L. Minard, M. Speranza, E. Agirre, I. Aldabe, M. van Erp, B. Magnini, G. Rigau, and R. Urizar, "SemEval-2015 Task 4: TimeLine: Cross-Document Event Ordering," in In Proceedings of the 9th International Workshop on Semantic Evaluation (SemEval 2015), no. SemEval, pp. 778-786, 2015.

[248] C. Manning, M. Surdeanu, J. Bauer, J. Finkel, S. Bethard, and D. McClosky, "The Stanford CoreNLP Natural Language Processing Toolkit," in In Proceedings of 52nd Annual Meeting of the Association for Computational Linguistics: System Demonstrations, pp. 55-60, 2015.

[249] "OpeNER suite of tools - ELRC-SHARE." https://elrc-share eu/repository/\{\%\}ODbrowse/opener-suite-of-tools/ \{\%\}0D1c0425724b2b11e9a7e100155d026706a830b480a23f40deb20750a2b6937309/. [Accessed: May-01-2020].

[250] "SENNA." https://ronan. collobert.com/senna/. [Accessed: May01-2020].

[251] S. Cornegruta and A. Vlachos, "Timeline extraction using distant supervision and joint inference," in EMNLP 2016 - Conference on Empirical Methods in Natural Language Processing, Proceedings, pp. 1936-1942, 2016.

[252] P. Raghavan, E. Fosler-Lussier, and A. M. Lai, "Inter-annotator reliability of medical events, coreferences and temporal relations in clinical narratives by annotators with varying levels of clinical expertise.," in 
Chapter 6. Conclusions and Future Work 6.2. Future Lines

AMIA ... Annual Symposium proceedings / AMIA Symposium. AMIA Symposium, vol. 2012, pp. 1366-74, 2012.

[253] T. Joachims, "Training Linear SVMs in Linear Time," in KDD '06 Proceedings of the 12th ACM SIGKDD international conference on Knowledge discovery and data mining, pp. 217-226, 2006.

[254] P. Raghavan, A. Lai, and E. Fosler-Lussier, "Learning to Temporally Order Medical Events in Clinical Text," in Proceedings of the 50th Annual Meeting of the Association for Computational Linguistics (Volume 2: Short Papers), no. July, pp. 70-74, 2012.

[255] P. Raghavan, E. Fosler-lussier, N. Elhadad, and A. M. Lai, "Crossnarrative temporal ordering of medical events," in Proceedings of the 52nd Annual Meeting of the Association for Computational Linguistics, pp. 998-1008, 2014.

[256] M. Straka, J. Hajic, J. Strakova, and J. Hajic Jr, "Parsing Universal Dependency Treebanks using Neural Networks and Search-Based Oracle," in 14th International Workshop on Treebanks and Linguistic Theories (TLT 2015), 2015.

[257] J. A. Strauss, C. R. Chao, M. L. Kwan, S. A. Ahmed, J. E. Schottinger, and V. P. Quinn, "Identifying primary and recurrent cancers using a SAS-based natural language processing algorithm," Journal of the American Medical Informatics Association, vol. 20, no. 2, pp. 349-355, 2013.

[258] E. Begoli and D. Kusnezov, "Artificial intelligence's essential role in the process of drug discovery," Future Drug Discovery, vol. 1, no. 2, p. FDD21, 2019.

[259] F. B. Maguire, C. R. Morris, A. Parikh-Patel, R. D. Cress, T. H. Keegan, C. S. Li, P. S. Lin, and K. W. Kizer, "A text-mining approach to obtain detailed treatment information from free-text fields in population-based cancer registries: A study of non-small cell lung cancer in California," PLoS ONE, vol. 14, no. 2, pp. 1-13, 2019.

[260] "Spring." https://spring.io/. [Accessed: 17-Jul-2019]. 
Chapter 6. Conclusions and Future Work 6.2. Future Lines

[261] "Enterprise Container Platform _ Docker." https://www.docker.com/ [Accessed: 17-Jul-2019]. 